\title{
Caveolin-1 recruitment to the trailing edge of motile cells results in focal adhesion disassembly and nascent interaction with actin stress fibers
}

Andrew Beardsley

West Virginia University

Follow this and additional works at: https://researchrepository.wvu.edu/etd

\section{Recommended Citation}

Beardsley, Andrew, "Caveolin-1 recruitment to the trailing edge of motile cells results in focal adhesion disassembly and nascent interaction with actin stress fibers" (2008). Graduate Theses, Dissertations, and Problem Reports. 2684.

https://researchrepository.wvu.edu/etd/2684

This Dissertation is protected by copyright and/or related rights. It has been brought to you by the The Research Repository @ WVU with permission from the rights-holder(s). You are free to use this Dissertation in any way that is permitted by the copyright and related rights legislation that applies to your use. For other uses you must obtain permission from the rights-holder(s) directly, unless additional rights are indicated by a Creative Commons license in the record and/ or on the work itself. This Dissertation has been accepted for inclusion in WVU Graduate Theses, Dissertations, and Problem Reports collection by an authorized administrator of The Research Repository @ WVU.

For more information, please contact researchrepository@mail.wvu.edu. 
Caveolin-1 Recruitment to the Trailing Edge of Motile Cells Results in Focal Adhesion Disassembly and Nascent Interaction with Actin Stress Fibers

Andrew Beardsley

Dissertation Submitted to the School of Medicine at West Virginia University

In Partial Fulfillment of the Requirements for the Degree of

Doctor of Philosophy in Physiology

\author{
Jun Liu, M.D Ph.D, Chair \\ Matthew Boegehold, Ph.D. \\ Vincent Castranova, Ph.D. \\ Daniel Flynn, Ph.D. \\ William Stauber, Ph.D. \\ Department of Physiology and Pharmacology \\ Morgantown, West Virginia \\ 2006
}

Keywords: caveolin-1; caveolae; focal adhesions; actin stress fibers; cell migration 


\title{
ABSTRACT \\ Caveolin-1 Recruitment to the Trailing Edge of Motile Cells Results in Focal Adhesion Disassembly and Nascent Interaction with Actin Stress Fibers
}

\author{
Andrew Beardsley
}

The protein caveolin-1 has been shown to positively affect angiogenesis and vascular remodeling in vivo via studies using knockout mice. In fact, defects in these two processes are among the major hallmarks of an otherwise benign caveolin-null phenotype. Current dogma on the function of caveolin-1 does not predict or account for these deficits. The overall objective of the following studies was to uncover the role of caveolin-1 in angiogenesis and vascular remodeling through study of the protein in cell-substratum remodeling during cell motility in vitro.

In the first study, caveolin-1 and its parent organelle, caveolae, conspicuously polarize to the rear of migrating human umbilical vein endothelial cells. Moreover, caveolin-1 localizated at the cell rear is mutually exclusive with focal adhesion staining and lamellipodial protrusion. Acute caveolin-1 knockdown by small, interfering RNA diminished the ability of endothelial cells to polarize and migrate toward a chemotactic stimulus.

In the second study, live cell imaging was used to study the dynamics between caveolin-1, focal adhesions, and the actin cytoskeleton. Caveolin-1 recruitment and transient association with focal adhesions at the trailing edge resulted in adhesion sliding and disassembly, concomitant with recoil of the trailing edge into the cell body proper. Moreover, association of caveolin-1 with actin stress fibers previously associated with adhesions in the collapsing trailing edge was observed. Mouse embryonic fibroblasts from caveolin-1 null mice demonstrated defects in trailing edge recoil compared to control cells with no decrease in cell contractility, suggesting a specific deficit in adhesion disassembly. Furthermore, caveolin-null cells displayed a decrease in overall chemokinetic motility and an increase in directional persistence, an indication that caveolin-1 contributes to movement plasticity via trailing edge focal adhesion disassembly.

In the final study, the interaction of polarized caveolin-1 with actin stress fibers at the cell rear was characterized. Caveolin-1 predictably associated with the cell perimeter depending on the direction of cell migration. Importantly, inhibition on non-muscle myosin by blebbistatin treatment abrogated initial polarization of caveolin-1, but did not affect caveolin-1 that had already polarized. Using live cell imaging in conjunction with photobleaching, actin-associated caveolin-1 was found to be extremely static upon polarization to the cell rear. In 
contrast, the initial polarization of caveolin-1 to retracting areas was highly dynamic. Furthermore, GM1 internalization at the cell rear was negligible, confirming that polarized caveolae are highly static. Forced disruption of the actin cytoskeleton by cytochalasin D treatment resulted in caveolin-1 depolarization and disaggregation into small puncta displaying frenetic, kiss-andrun movement. Furthermore, cytoskeletal remodeling in response to change in direction of a cell resulted in similar caveolin depolarization.

In summary, stress fibers associate with and exert traction on trailing edge focal adhesions during cell motility. This traction force is prerequisite for caveolin-1 recruitment. Arrival and transient association of caveolin-1 with focal adhesions results in adhesion disassembly and stable interaction of caveolin with actin stress fibers. Thus, a novel mechanism in cellular mechanotransduction can be described, whereby cells utilize caveolin-1 recruitment to relieve strain generated at the cell perimeter by the actin cytoskeleton during movement. This novel function of caveolin-1 may analogously occur in vivo, beyond the context of endothelial cell migration. The deficits in angiogenesis and vascular remodeling seen in caveolin-1 null mice might thus be explained by the role of caveolin-1 in cell-substratum remodeling in response to strain. 


\section{Acknowledgements}

If it isn't evident in my writing, I feel very strongly that our lab has made a key (and really cool) discovery in cell biology. For my part, I am indebted to Jun's didactic approach as a mentor. He did not force me to adhere stringently to the dogmas in our field. My interpretation of data was therefore de novo-1 was seeing with my own eyes. I believe his openness of thought is a great attribute in a mentor.

It's amazing how the project has evolved over the last four years. My favorite memories are the dozens of hours of thought experiments at the dry erase board in Jun's office. The free exchange of ideas and interpretations of data were indispensable to our progress. Thanks, Jun.

I need to thank my co-workers, Wei, Xinghui, Kelly, and Kai. There is no question of the synergy at work in the lab. I hope you feel the same. Good luck and I hope you each publish several thousand papers and become vastly wealthy throughout your career.

Thanks to Dr. Lyndell Millecchia at NIOSH. I could not have finished the project without her.

Thanks to Mom, Dad, Lis, and Katie for support during the many vicissitudes of the project. Thanks also to Paul Marvar for listening to my sometimes incessant complaining about research etc. Thanks especially to Kristina, who has heard quite enough about caveolin.

I would like to dedicate this dissertation to the memory of Dr. Franz van Damme, a talented glassworker who once made a fully functional trombone out of borosilicate glass. 
Abstract.....................................................iii

Acknowledgements................................... iv

Table of Contents.......................................

List of Figures...........................................vii

Glossary ..............................................ii

Literature Review........................................... 1-31

Caveolin/Cavelae

Focal Adhesions

Cell Motility, Adhesions, and Caveolin

Study 1: Loss of Caveolin-1 Polarity affects Endothelial Cell Polarization and Directional Movement. 32-65
Abstract
Introduction
Methods
Results
Discussion

Study 2: Caveolin-1 Recruitment Promotes Focal Adhesion Disassembly at the Trailing Edge of Motile Cells. .66-101
Abstract
Introduction
Methods
Results
Discussion

Study 3: Characterization of Caveolin-1 Anchorage to Actin Stress Fibers at the Cell Trailing Edge.

Abstract

Introduction

Methods

Results

Discussion

General Discussion $137-143$

General References. $144-160$ 


\section{List of Figures}

\section{Study 1}

1. Caveolin-1 polarization during cell migration.

2. Mutual exclusion between caveolin-1 and focal contacts at the leading edge.

3. Exclusion of caveolae from lamellipodia.

4. Co-localization of phospho-caveolin-1, but not caveolin-1, with focal complex molecules at leading edge of migrating cells.

5. Knockdown of caveolin-1 prevents endothelial cell polarization.

6. Knockdown of caveolin-1 inhibits endothelial cell directional movement.

7. Proposed model demonstrating caveolin-1 polarity and control of lamellipod protrusion.

Study 2

1. Caveolin-GFP colocalizes with endogenous caveolin-1 at the cell rear.

2. Polarization of caveolin-1 occurs during cell polarization.

3. Caveolin-1 recruitment precedes the recoil of trailing fibers during cell migration.

4. Caveolin-1 recruitment leads to focal adhesion disassembly and sliding in the trailing edge/ Analysis of trailing adhesion disassembly as a function of caveolin-1 distance.

5. Involvement of the actin cytoskeleton in trailing edge recoil.

6. Impaired focal adhesion disassembly at cell trailing edges in $\mathrm{Cav}^{-/-} \mathrm{MEFs}$.

7. Analysis of chemokinetic motility in mutant and control cells.

8. Directional Persistence is increased in caveolin-1 null MEFs.

Study 3

1. Caveolin-1 and CTB-TRITC accumulation at the cell perimeter occurs predictably with cell movement.

2. Initial recruitment of caveolin-1 to the cell perimeter is dependent on cell contractility.

3. Caveolin-1 and actin stress fibers stably co-align at the cell rear.

4. Photobleaching analysis of polarized caveolin-1. 
5. Incorporation of caveolin-1-GFP fluorescence in retracting areas of the trailing edge.

6. CTB-TRITC labeled GM1 is stable at the cell perimeter.

7. Disruption of caveolin-1 polarization via cytoskeletal disruption.

8. Lamellipodial outgrowth from the cell rear results in caveolin-1 and actin stress fiber dispersion. 


\section{Glossary}

BAEC bovine aortic endothelial cell

bFGF basic fibroblast growth factor

Cav-1 caveolin-1

Cav-2 caveolin-2

Cav-3 caveolin-3

Cav-

GFP caveolin-green fluorescent protein

CSD caveolin scaffolding domain

Csk c-terminal src kinase

CTB cholera toxin subunit B

DGC dystrophin-glycoprotein complex

EGF epidermal growth factor

EGFP enhanced green fluorescent protein

eNOS endothelial nitric oxide synthase

FAK focal adhesion kinase

FBS fetal bovine serum

FLIP fluorescence loss in photobleaching

FRAP fluorescence recovery after photobleaching

HUVEC human umbilical vein endothelial cell

IR- $\beta$ insulin receptor beta

MAP mitogen activated protein

MDCK Madin-Darby canine kidney epithelial cell

MEF mouse embryonic fibroblast

MLCK myosin light chain kinase

NBCS newborn calf serum

NRTK non-receptor tyrosine kinase

$\mathrm{PKCa}$ protein kinase $\mathrm{C}$ alpha

PYK2 proline-rich tyrosine kinase 2

RFP red fluorescent protein

siRNA small, interfering ribonucleic acid

TRITC tetramethyl rhodamine isothiocyanate

VEGF vascular endothelial growth factor 


\section{Literature Review}

Caveolae (L. little caves) are plasma membrane invaginations described in continuous endothelium and initially dubbed plasmalemmal vesicles by the electron microscopist George Palade (Palade 1953). Yamada later identified analogous structures in gall bladder epithelium, naming them caveolae intracellulares, as they appeared as little caves recessed from the cell membrane (Yamada 1955). After initial identification in epithelium, caveolae were subsequently detected in most cell types, excluding certain cells of hematopoietic lineage such as erythrocytes, platelets, and lymphocytes (Fra, Williamson et al. 1995), as well as some neuronal cells (Gorodinsky and Harris 1995). Until the discovery of the caveolae-associated protein, caveolin-1, nearly forty years after these initial descriptions, caveolae were solely studied ultrastructurally, identified by morphology per se in electron micrographs. Not surprisingly, much early research on these membranous invaginations, which appear to be pinching off from the cell membrane, prescribed a role for caveolae in endocytosis and transcytosis, or movement of molecules (e.g. folate) across endothelial cells (Anderson, Kamen et al. 1992). Notably, numerous morphological studies have demonstrated that caveolae are not randomly distributed in the cell membrane, but exist as longitudinal arrays tethered to the filamentous actin cytoskeleton (Simionescu, Simionescu et al. 1974; Gabella 1978; Sawada, Ishikawa et al. 1978; Peters, Carley et al. 1985; Izumi, Shibata et al. 1988). Moreover, caveolae, in contrast to endocytically-active clathrin-coated pits, have strikingly 
monotonous morphologies, with an average outer diameter of 70nm (Bruns and Palade 1968; Bruns and Palade 1968; Palade and Bruns 1968; Stan 2005).

The next advent in caveolae biology was the identification of a 21-22 kD tyrosine-phosphorylated substrate in chick embryo fibroblasts transformed by vSrc oncogene by Glenney (Glenney 1989). This protein was later discovered to be a major protein component of caveolae and consequently named caveolin by Anderson in 1992 (Rothberg, Heuser et al. 1992). Simons independently reported that VIP21, later found to be identical to caveolin, was the major protein component in detergent-insoluble plasma membrane microdomains enriched in glycosphingolipids and cholesterol (Kurzchalia, Dupree et al. 1992). Caveolae were thus characterized for the first time beyond morphological criteria, enriched in caveolin and with a specific, relatively detergent-insoluble lipid composition. Detergent insoluble microdomains are present in cells lacking caveolin, and thus caveolae have been considered as a subclass of lipid rafts, ordered lipid microdomains enriched in cholesterol and sphingolipids that self-assemble and "float" in the more fluid, less-ordered phospholipid "sea". The biophysical characteristics conferred by the unique lipid environment of lipid rafts/caveolae have allowed investigators to isolate such membrane microdomains and identify resident proteins, leading to the hypothesis that such domains may serve as hubs of signal transduction (see below).

Since the discovery of the protein caveolin by Glenney, three related genes, well-conserved among amniotes (Stan 2005), and encoding at least six different isoforms, have been identified. CAV-1 encodes the 178-residue Cav-1 $\alpha$, 
the protein originally identified in transformed chick embryo fibroblasts, as well as the 147-residue Cav-1 $\beta$ (Scherer, Tang et al. 1995). These isoforms are translated from different mRNAs, the latter derived from an internal translation initiation site. Cav-2, first described in caveolae-enriched adipocyte membranes, consists of three isoforms encoded by $C A V-2$ : Cav-2 $\alpha$, the full length protein, and two uncharacterized forms arising from alternative splicing, Cav-2 $\beta$ and Cav$2 y$ (Scherer, Okamoto et al. 1996). Finally, CAV-3, identified through database searches and screening of cDNA libraries for genes with sequence homology to CAV-1, encodes the 151-residue, muscle-specific Cav-3 (Tang, Scherer et al. 1996). All three genes are located on the murine chromosome 6, while human $C A V-1$ and $C A V-2$ are found on chromosome locus 7q31.1 (Engelman, Zhang et al. 1998; Engelman, Zhang et al. 1998); CAV-3 maps to chromosome locus 3p25 (McNally, de Sa Moreira et al. 1998; Minetti, Sotgia et al. 1998). The expression patterns of Cav-1 and Cav-2 overlap, and parallel the tissue distribution of caveolae, with highest expression in terminally-differentiated cells such as endothelium, fibroblasts, and adipocytes (Scherer, Okamoto et al. 1996; Razani, Wang et al. 2002). As mentioned, Cav-3 is regarded as the muscle specific isoform, expressed in skeletal muscle, myocardium, and smooth muscle, although it has also been shown to be expressed in astrocytes and sinus endothelial cells (Ikezu, Ueda et al. 1998; Uehara and Miyoshi 2002).

Originally identified as a phosphoprotein, Cav-1a is phosphorylated on tyrosine 14 by $\mathrm{v}$-src, as well as the nonreceptor tyrosine kinases c-src, Abl and Fyn. The significance of Cav-1 phosphorylation is not clearly understood, but a 
recent study using a modified yeast two-hybrid system has identified C-src terminal kinase (Csk) as a specific binding partner for phosphorylated caveolin, which the investigators proposed as a negative-feedback loop in src family kinase signaling (Ikezu, Ueda et al. 1998; Uehara and Miyoshi 2002). In addition, Cav-1 is phosphorylated on serine/threonine sites by PKCa, although the significance of this phosphorylation is not well-understood. Phosphorylation of serine residues 23 and 36 is on Cav-2 is thought to regulate Cav-1-dependent caveolae formation (Sowa, Pypaert et al. 2003). Moreover, the phosphorylation of Cav-2 either on tyrosine residue 19 or 27 , resulting in distinct cellular localizations of the protein, has been recently been documented (Wang, Lee et al. 2004).

Cav-1 topology is unique in that the molecule lacks an N-terminal signal sequence, but rather has a single, central hydrophobic domain (residues 102-134) responsible for membrane insertion and association with the endoplasmic reticulum upon translation. Thus both the $\mathrm{N}$ and $\mathrm{C}$ termini of Cav-1 remain cytoplasmic, resulting in a hairpin configuration of the molecule. Cav-1 can form homo-oligomers through mutual interaction between residues $61-101$, known as the oligomerization domain (Sargiacomo, Scherer et al. 1995; Song, Tang et al. 1997). Moreover, although Cav-2 cannot form large oligomers per se, it can interact with Cav-1 via their respective membrane spanning domains (Das, Lewis et al. 1999). Cav-1 and Cav-2 are thought to assemble in 15-mers at the endoplasmic reticulum shortly after synthesis and before exit to the Golgi apparatus (Scherer, Lewis et al. 1997). Post-translational palmitoylation of 
cysteine residues at the C-terminus occurs in Cav-1 and Cav-3. Unlike reversible and regulated acylation of other proteins, this palmitoylation is irreversible, and has been suggested to stabilize caveolin oligomers (Monier, Parton et al. 1995; Dunphy and Linder 1998; Parat and Fox 2001). Such palmitoylation has been demonstrated to be dispensable in caveolin membrane targeting and recruitment to caveolae (Parat, Anand-Apte et al. 2003).

Several critical experiments reveal that caveolin, along with cholesterol, is essential in the formation of caveolae. Heterologous expression of Cav-1a or Cav-1 $\beta$ in cells (e.g. lymphocytes) lacking both caveolin and caveolae is sufficient to induce caveolae formation (Fra, Williamson et al. 1995). In contrast, caveolae formation does not occur upon ectopic expression of Cav-2, which instead accumulates in the Golgi apparatus. Significantly, co-expression of Cav1 rescues Cav-2 from Golgi sequestration, allowing it to shuttle to caveolae at the cell membrane (Mora, Bonilha et al. 1999; Parolini, Sargiacomo et al. 1999). Confirming a role for the caveolins in caveolae formation, mice lacking the CAV-1 or $C A V-3$ gene demonstrate a complete loss of caveolae from tissues in which the genes are normally expressed (Drab, Verkade et al. 2001; Razani, Engelman et al. 2001; Woodman, Park et al. 2002). Predictably, caveolae were detected in the muscle of $C A V-1$-/- mice; and in the endothelium, type 1 pneumocytes, fibroblasts, and adipocytes of CAV-3 -/- mice. No caveolae have been detected to date in CAV-1/CAV-3 double knockout mice (Park, Woodman et al. 2002). Consistent with prior results, mice in which CAV-2 has been deleted demonstrate a comparable number of caveolae to wild type control mice (Razani, Wang et al. 
2002), indicating that Cav-2 is not essential in caveolae genesis. Finally, cholesterol is indispensable for the characteristic flask-shaped curvature of caveolae, as depletion by nystatin results in caveolar "flattening", evidenced by concentration of caveolin in patches at the plasma membrane by immuno-EM (Rothberg, Heuser et al. 1992).

The contribution of caveolin proteins to the formation of caveolae has not been determined. Interestingly, the presence of ridges, or a "coat", decorating the cytoplasmic face of caveolae has been described in numerous morphologic studies (Izumi, Shibata et al. 1988; Izumi, Shibata et al. 1989; Izumi, Shibata et al. 1991). Moreover, Anderson demonstrated by immunogold labeling that Cav-1 was localized on the caveolar ridges, and postulated that the oligomerized protein is the structural element of the coat, analogous to the role of clathrin in clathrin-coated pits (Rothberg, Heuser et al. 1992). Stan challenged this assertion, noting that myosin fragments could decorate the coat ridges, making them more prominent, and suggests a myosin interacting protein (i.e. actin) was contributing to the caveolar coat. Consistent with this, pretreatment with phalloidin, an actin-stabilizer, enhanced the appearance of the caveolar coat (Stan 2005). Notably, Izumi et al. failed to detect the caveolar ridges in adipocytes, indicating that such a coat is not universally present in caveolae, and casting doubt on whether the coat is a structural determinant of caveolar shape (Izumi, Shibata et al. 1989). Caveolae have long been known to associate with the actin cytoskeleton, and an interaction between Cav-1 and actin via filaminA has been described (Stahlhut and van Deurs 2000). Thus, it is plausible that 
Cav-1 coexists with actin and linking proteins in the caveolar coat, although the significance of the coat is not known.

As mentioned, the characteristic curvature of caveolae suggests imminent budding from the plasma membrane, leading investigators to study the role of caveolae, and more recently, caveolin, in endocytosis and cellular trafficking. Whether or not caveolae/caveolin function significantly in endocytosis and transcytosis is controversial, however, and lack of a consensus illustrates the general confusion about the function of caveolar microdomains. Numerous morphological studies have implicated endothelial caveolae in the transcytosis of varying blood-borne macromolecules (e.g. albumin and LDL) (Anderson 1998). Moreover, a study using cholesterol depletion to destroy caveolae resulted in decreased uptake of albumin in cultured endothelial cells (John, Vogel et al. 2001), seeming to confirm the morphological evidence, and leading to the assertion that caveolae are the major endocytic compartment of endothelial cells (Shajahan, Timblin et al. 2004; Shajahan, Tiruppathi et al. 2004). Investigation of albumin transcytosis in caveolin-1 null mice has provided conflicting results regarding the hypothesis that caveolae-mediated transcytosis is the major route of albumin transport across the endothelium. Drab et al. reported that the cerebrospinal fluid albumin concentration was normal in caveolin-1 deficient animals, suggesting no deficit of albumin transcytosis across the endothelium (Drab, Verkade et al. 2001). Later, Schubert et al. reported that, in contrast to wild type controls, gold-conjugated albumin is not cleared from the vascular lumen by endothelial cells in caveolin-1-null mice (Schubert, Frank et al. 2001). 
In the same study, uptake of radioiodinated albumin was similarly negligible in caveolin-1-null aortic ring segments compared to control. Paradoxically, Schubert et al. next published a study in which clearance of radioiodinated albumin from the circulatory system was found to be substantially elevated caveolin-1-null mice compared to wild-type controls (Schubert, Frank et al. 2002). Although the authors suggest an increase in paracellular transport due to dysregulation of eNOS (see later), the result stands in contrast to the earlier report that albumin is less efficiently cleared from the blood in knockout mice. Increased transendothelial transport of radioiodinated albumin in caveolin-1-null mice was reported that weakened the claim that albumin accumulates in the vessel lumens of these mice, and also the hypothesis that caveolae is essential in the clearance of albumin from the vascular compartment (Rosengren, Rippe et al. 2006).

Endocytosis of cholera toxin subunit B (CTB), which binds to the caveolae-enriched sphingolipid, GM1, has been presumptively studied as a specific marker of caveolae-dependent internalization (Shajahan, Timblin et al. 2004; Shajahan, Tiruppathi et al. 2004; del Pozo, Balasubramanian et al. 2005). Although the potential for CTB endocytosis by caveolae has been demonstrated (Yao, Chen et al. 2005), many lines of evidence refute the claim that such internalization is critically dependent on a caveolar route. Importantly, cells devoid of caveolae internalize cholera toxin, and expression of caveolin in such cells does not increase or decrease this uptake (Torgersen, Skretting et al. 2001). Similarly, murine embryonic fibroblasts derived from caveolin-1 knockout mice 
demonstrated CTB internalization comparable to control cells, confirming caveolae-independent entry (Kirkham, Fujita et al. 2005). Surprisingly, even the role of caveolin-1 in putative caveolar endocytosis is contested. Le et al. have reported that overexpression of the protein actually blocks albumin and CTB uptake (Le, Guay et al. 2002), leading to the idea that caveolin-1 actually antagonizes caveolar budding from the cell membrane. Through photobleaching studies on chimeric caveolin-green fluorescent protein (Cav-GFP), van Deurs et al. have demonstrated that caveolin-1 is highly immobile at the plasma membrane in vitro (Thomsen, Roepstorff et al. 2002). This result was recently confirmed by total internal reflection fluorescence microscopy of Cav-GFP, demonstrating highly immobile caveolae in the ventral plasma membrane (Tagawa, Mezzacasa et al. 2005). These reports have led to the assertion that caveolae are largely static at the cell surface, and thus generally not involved in constitutive endocytosis. Tagawa et al. recently reported that incubation with the activated SV40 resulted in mobilization of static Cav-GFP signal, and concluded that caveolae internalization, though generally dormant, might be cargo-triggered (Tagawa, Mezzacasa et al. 2005). Thus little consensus exists on the role of caveolae or caveolin-1 in endocytosis. Nevertheless, a burgeoning amount of evidence indicates that caveolae are not primarily or constitutively involved in endocytosis.

Similar confusion exists regarding another putative function of caveolin$1 /$ caveolae, the regulation of signal transduction. The discovery of caveolin as a caveolar marker protein allowed the subsequent purification and investigation of 
detergent resistant caveolae membranes (DRMs). Such membranes were found to be enriched in a myriad of signaling molecules, including GPI-anchored proteins, endothelial nitric oxide synthase (eNOS), and receptor and nonreceptor tyrosine kinases (Sargiacomo, Sudol et al. 1993; Garcia-Cardena, Oh et al. 1996; Shaul, Smart et al. 1996; Liu, Oh et al. 1997). Caveolae were thus suggested to compartmentalize, modulate, and/or integrate signaling at the cell membrane (Shaul and Anderson 1998). Indeed, early research established that caveolae were critically involved in the organization of several signaling events at the cell membrane in various cell lines (Liu, Ying et al. 1997; Labrecque, Royal et al. 2003). The viability of caveolin knockout mice, however, suggests that caveolar regulation of signaling is dispensable for life. Moreover, the dubious purity of isolated caveolae membranes has led investigators to question whether proteins that separate with these membranes actually associate with caveolae in vivo. Nevertheless, specific dysregulation of eNOS activity, a prominent phenotype of caveolin null mice, and supports a definitive role for caveolin/caveolae in the modulation of agonist-induced eNOS signaling.

eNOS is a membrane-associated NOS isoform that is N-myristoylated at glycine 2 and palmitoylated at cysteine 15 and 26 (Fulton, Gratton et al. 2001; Sessa 2005). The co- and post-translational modifications were found to be critical in targeting the enzyme to caveolae/lipid rafts at the cell membrane (Shaul, Smart et al. 1996). In addition to its localization in caveolae membranes, eNOS was found to directly interact with caveolin-1 (Feron, Belhassen et al. 1996; Ju, Zou et al. 1997), resulting in tonic inhibition of nitric oxide production. Later, it 
was shown that calmodulin disrupted caveolin-1-eNOS interaction by allosteric competition in a $\mathrm{Ca}^{2+}$-dependent fashion (Michel, Feron et al. 1997), prompting release from caveolae and subsequent activation of eNOS.

The negative effect of caveolin-1 on eNOS activity has been demonstrated in several in vivo models. eNOS-dependent blood vessel relaxation in response to acetylcholine stimulation was abrogated upon exposure of the so-called caveolin scaffolding domain (CSD), comprised of caveolin-1 residues 82-101 and demonstrated to be sufficient for eNOS inhibition in vitro (Bucci, Gratton et al. 2000). CSD exposure had no effect on relaxation in response to sodium nitroprusside or prostacyclin release, indicating that the CSD specifically inhibits eNOS. In addition, basal and acetylcholine-stimulated eNOS activities were shown to be elevated in aortas from caveolin-null mice (Drab, Verkade et al. 2001; Razani, Engelman et al. 2001). Moreover, the knockout animals display microvascular hyperpermeability, which is reversed by treatment with the eNOS inhibitor L-NAME (Schubert, Frank et al. 2002). Thus, considerable lines of evidence support the role of caveolin/caveolae in the tonic inhibition of eNOS activity.

Several phenotypes of CAV-1 knockout mice (i.e., lack of caveolae, albumin transcytosis, and enhanced eNOS activity) have been discussed. The generation of viable and fertile caveolin-1 null mice by four independent labs represents the most important advance in the field since the discovery of caveolin. The viability of the mice was surprising to many, given the considerable evidence that caveolin served essential and myriad cellular 
functions (Anderson 1998). Indeed, several aspects of the caveolin-null phenotype ostensibly negate or challenge previous assertions about the protein. As discussed, the role of caveolae/caveolin in the transcytosis of blood-borne proteins cannot be considered essential. Likewise, endocytosis of CTB as well as Simian Virus 40, both described to internalize via caveolae (Pelkmans, Kartenbeck et al. 2001; Yao, Chen et al. 2005), still occurs by caveolaeindependent pathways in caveolin-1 null cells (Damm, Pelkmans et al. 2005; Kirkham, Fujita et al. 2005). Moreover, caveolin-1 ablation had no effect on the targeting of GPI-anchored proteins to DRMs, underscoring the unreliability of DRM co-fractionation in predicting caveolar localization of a given protein (Drab, Verkade et al. 2001). Furthermore, an assertion in several reports that flaskshaped caveolae can exist in the absence of caveolin (Le, Guay et al. 2002; Parat, Anand-Apte et al. 2003) is doubtful, considering the absolute lack of caveolae in caveolin-null mice. On the other hand, the multiple phenotypic aberrations present in caveolin-null mice provide a clearer picture of caveolin function, and shall be discussed in turn.

As previously mentioned, $C A V-1$ knockout eliminates caveolae biogenesis in non-muscle tissues. Additionally, loss of caveolin-1 results in a decrease of caveolin-2 protein to less than $10 \%$ of wild-type levels (Drab, Verkade et al. 2001; Razani, Engelman et al. 2001), despite normal transcription levels. It thus appears that caveolin-1 is necessary for arrangement of caveolin-2 into heteromultimers and subsequent trafficking to the plasma membrane. Caveolin2 retained in the Golgi in the absence of caveolin-1 undergoes proteolytic 
degradation. Interestingly, treatment of cells lacking caveolin-1 with proteasomal inhibitors not only restores caveolin-2 protein to near-wild-type levels, but also results in normal membrane targeting (Mora, Bonilha et al. 1999; Parolini, Sargiacomo et al. 1999), indicating that caveolin-2 not associated with caveolin-1 may normally be "mopped up" in the Golgi by proteolytic degradation.

CAV-1-/- mice are metabolically deranged, displaying basal hypertriglyceridemia and elevated free fatty acids, with no changes in insulin, glucose, or cholesterol levels under normal circumstances (Razani, Combs et al. 2002; Le Lay and Kurzchalia 2005). Young CAV-1-/- mice totally lack the hypodermal fat layer, despite histologically normal intraperitoneal fat pads (Razani, Combs et al. 2002; Hnasko and Lisanti 2003). The animals appear lean as they age, however, and abdominal fat pads are clearly smaller than wild-type animals. This difference becomes especially striking when the mice are challenged by a high-fat diet. Histologically, the caveolin-1-null adipocytes from the fat pads appear smaller than their wild-type counterpart.

Despite normal resting levels of insulin, CAV-1-/- mice display insulin resistance, and develop postprandial hyperinsulinemia in response to a high-fat diet (Cohen, Combs et al. 2003; Cohen, Razani et al. 2003). This abnormality was attributed to reduction of insulin-receptor (IR- $\beta$ ) in adipose tissue to less than $10 \%$ of wild-type levels, despite normal transcription. Exogenous expression of caveolin-1 in CAV-1-/- mouse embryonic fibroblasts results in restoration of IR- $\beta$ to wild-type levels, confirming an earlier report that caveolin-1 stabilizes insulin receptor at the cell membrane (Yamamoto, Toya et al. 1998). 
CAV-1-/- mice display marked urogenital system abnormalities with age. By five months, around two-thirds of male knockouts develop urinary calcium stones compared to $20 \%$ in wild type controls (Cao, Yang et al. 2003; Woodman, Cheung et al. 2004). Defective calcium reabsorption was attributed to mislocalization of the pump protein PMCA in the distal nephron. Moreover, aged knockout mice develop bladder smooth muscle thickening coincident with increased bladder pressures (Woodman, Cheung et al. 2004). These changes are accompanied by fluid accumulation in the prostate and seminal vesicles, causing dramatic enlargement of these tissues (Le Lay and Kurzchalia 2005), as well as vacuolization in the kidneys (Hnasko and Lisanti 2003).

Female CAV-1-/- mice display accelerated mammary gland development during pregnancy, leading to premature lactation (Park, Lee et al. 2002; Hnasko and Lisanti 2003). Caveolin-1 is thought to negatively modulate prolactin signaling by sequestering the Janus kinase, Jak-2, thus preventing prolactininduced phosphorylation of STAT5a (Clevenger and Kline 2001). Thus, caveolin ablation results in dysregulation of the prolactin pathway, resulting in the reported acceleration in mammary gland development.

CAV-1-/- mice display a number a cardiovascular abnormalities, including dysregulated eNOS activity (already discussed), endothelial hypercellularity in the lungs, pulmonary hypertension, dilated cardiomyopathy, and cardiac hypertrophy (Drab, Verkade et al. 2001; Razani, Engelman et al. 2001). Besides dysregulation of eNOS, these cardiovascular manifestations are phenocopied in $C A V-2-/-$ mice, strongly suggesting that loss of caveolin-2 contributes to the 
abnormalities (Razani, Wang et al. 2002). Lungs from both CAV-1-/- and CAV-2/- demonstrate alveolar thickening, endothelial hypercellularity, upregulation of VEGF-R2, a marker of non-differentiated endothelial cells, and lack of von Willebrand factor, a marker of differentiated endothelium (Razani, Wang et al. 2002). In the first report on the caveolin-2 null phenotype, Razani et al. point out that this vascular hypercellularity is analogously encountered in fetal lung parenchyma as a reaction to increased mechanical stress and strain intrinsic to the breathing process (Liu, Tanswell et al. 1999), and suggest that lung caveolae may function in mitigating such mechanical forces in adulthood. Thus, in the absence of caveolae/caveolin, the inability of the pulmonary vasculature to adapt to shear stresses of blood arriving from the right heart may result in a compensatory proliferation of endothelial cells. Consistent with this hypothesis, Yu et al. have demonstrated that endothelium lacking caveolin was unable to couple changes in blood flow with proportional vascular remodeling in intact and isolated vessels (Yu, Bergaya et al. 2006). Instead, changes in blow flood resulted in endothelial cell proliferation and wall thickening.

The altered lung phenotype observed in CAV-1-/- and CAV-2-/- mice is suggestive of restrictive lung disease; accordingly, these animals are dramatically exercise-intolerant. Moreover, the development of right heart failure has been described in CAV-1-/- animals (Drab, Verkade et al. 2001; Razani, Engelman et al. 2001). As cardiac myocytes do not express caveolin-1, the heart failure observed in caveolin-1 null mice could presumably occur subsequently to 
the development of pulmonary hypertension. Curiously, cardiac disease has yet to be reported in CAV-2-/- animals.

Angiogenesis, the growth of new blood vessels from preexisting capillary beds, has been reported to be impaired in CAV-1-/- mice. Woodman et al. reported impaired endothelial cell invasion into bFGF-impregnated Matrigel plugs implanted in CAV-1-/- mice compared to wild-type control (Woodman, Ashton et al. 2003). Moreover, B16-F10 melanoma cells injected into CAV-1-/- mice were decreased in size and vessel density. Sonveaux et al. reported that femoral artery resection in CAV-1-/- mice did not result in recovery of a functional vasculature as in wild type mice, which instead lost part of the ligated limb as a consequence of ischemia (Sonveaux, Martinive et al. 2004). Thus, caveolin/caveolae seem to play critical roles in certain models of adult angiogenesis.

As mentioned, CAV-2-/- mice develop pulmonary disease which is indistinguishable from CAV-1-/- mice; however, caveolin-2 ablation does not result in vascular system eNOS dysfunction or metabolic derangements present in CAV-1-/- mice (Razani, Wang et al. 2002). To date, there are no reports on the effect of caveolin-2 ablation on angiogenesis, although such a study is certainly warranted. Targeted deletion of caveolin-3, the muscle-specific caveolin isoform, results in muscle degeneration, cardiomyopathy, and T-tubule disorganization (Hagiwara, Sasaoka et al. 2000; Galbiati, Engelman et al. 2001). Muscle degeneration in these mice was anticipated, as several dominantnegative mutations in caveolin-3 have been described in human muscular 
dystrophies (Minetti, Sotgia et al. 1998; Woodman, Sotgia et al. 2004). Caveolin3 is thought to play a role in the maintenance of the structural integrity of skeletal muscle through interaction with the dystrophin-glycoprotein complex (DGC) (Halayko and Stelmack 2005), although the nature of such an interaction has not been elucidated.

The fact that caveolin null animals are viable and fertile is an implicit argument against the notion that caveolin/caveolae critically and generally organize cell signaling at the plasma membrane. Moreover, the failure of these animals to develop spontaneous tumors weakens a previous assertion that caveolin is a potent tumor suppressor protein (Razani, Schlegel et al. 2001). Although caveolae have been studied in transcytotic transport for decades, several lines of data cast doubt on whether this is indeed a constitutive function of caveolae (see above). The knockout phenotypes thus suggest that earlier dogma about caveolin function may have considerably missed the mark. One emergent theme in the caveolin-null phenotypes is the aberrant adaptation to environmental and developmental stimuli in the absence of caveolin. For instance, normal vascular remodeling in response to changes in shear rate is replaced by a paradoxical hyperproliferative response in caveolin-1-null endothelial cells. Hypercellularity is also observed in bladders of aged CAV-1-/mice; this phenomenon, coincident with an increase in bladder tone, could analogously be explained by compromised developmental remodeling in the face of caveolin ablation. Similarly, muscle degeneration in CAV-3-/- mice could potentially be explained by the relative inability of caveolin-3 null myocytes to 
adapt during development. Since these cells do not have the option to proliferate, they may rather succumb to apoptosis or necrosis, eventually resulting in muscle degeneration.

Despite such evidence, little to no data or even conjecture exists regarding the role of the caveolin gene family in the development of an organism. On a cellular level, the interplay between cytoskeletal elements critically and dynamically determines cell shape; remodeling of the cytoskeleton in response to stimuli can be considered a priori a principle component of developmental adaptation in vivo. Donald Ingber has keenly likened such interplay of different cytoskeletal elements to the tensegrity sculptures of Kenneth Snelson, composed of isolated steel beams interconnected with a continuous series of tensed metal cables (Ingber 1997; Ingber 1997). These sculptures stand freely in three dimensions, stabilized mechanically by the interplay of tension and compression forces, a phenomenon termed "tensegrity" by Buckminster Fuller. Such tensegrity, or prestress, analogously exists in the development, maintenance, and modification of cell shape. Specifically, the core actin cytoskeleton, composed of bundles of filamentous actin (F-actin), serves as a contractile force in conjunction with myosin and in analogy to the tensed steel cables in tensegrity sculptures. On the other hand, focal adhesions, which are discreet points of anchorage between the ventral plasma membrane and the substratum, serve to resist the pull of the actin filaments, in analogy to the compressed steel beams in Snelson's sculptures. Ingber proposed that microtubules additionally function to resist the pull of the actin cytoskeleton (Mustata and Rusu 1998), and the net 
effect of push and pull between these several components is a discreet cellular morphology stabilized by prestress, or tensegrity.

As mentioned, cytoskeletal remodeling is undoubtedly involved in the development of an organism at the cellular level. How a cell maintains a healthy level of prestress throughout cytoskeletal remodeling necessitated by growth and development is a fascinating question, partially addressed by the discipline of cellular mechano-transduction through analysis of focal adhesion and cytoskeletal dynamics. The molecular composition of focal adhesions and their linkage with the actin cytoskeleton will presently be described, followed by an overview of adhesion dynamics during cell migration, an accessible model of cytoskeletal remodeling. Finally, current data linking caveolin-1 to the actin cytoskeleton and focal adhesion turnover, suggestive of a putative role for the protein in mechano-transduction, shall be considered.

Focal adhesions, likened by investigators to spot welds (Ingber 1997), are discreet sites of anchorage between a cell and its substrate (Burridge and Chrzanowska-Wodnicka 1996). Clustering of cell-surface glycoproteins known as integrins is the nidus for focal adhesion formation. Integrins exist as heterodimers, consisting of an $\alpha$ and $\beta$ subunit, each containing a large, extracellular domain, a single membrane-spanning sequence, and usually a short cytoplasmic domain (Palecek, Huttenlocher et al. 1998). The integrin receptor gene family of vertebrates consists of at least 16 and $8 \alpha$ and $\beta$ subunits, respectively, which can form at least 20 distinct heterodimers with differing ligand affinities (Hynes 1992; Aplin, Howe et al. 1998). For instance, the integrin 
heterodimer $\alpha 5 \beta 1$ is the "classic" fibronectin receptor, binding to an arginineglycine-aspartic acid (RGD) peptide in a specific domain of the fibronectin molecule (Aplin, Howe et al. 1999), whereas integrin av $\beta 3$ more promiscuously binds collagen VI, laminin, fibronectin, vitronectin, thrombospondin, and others (Kuhn and Eble 1994). Integrin heterodimers mediate cell-substrate adhesion through interaction of the extracellular domain with matrix proteins. This interaction is dependent on divalent cation binding to an $\mathrm{N}$-terminal portion of the a subunit designated "MIDAS", or metal-ion dependent adhesion site (Loftus and Liddington 1997). Conformational changes in the integrins (e.g., in response to divalent cation binding) can switch the molecule from a low- to high-affinity state and vice-versa with respect to ligand binding. Interestingly, deletion of highly conserved sequences in the membrane proximal cytoplasmic domain in either subunit constitutively "locks" the integrin in a high affinity state (O'Toole, Katagiri et al. 1994; Tozer, Hughes et al. 1996). Moreover, activation of integrins by ligand binding is thought to cause the $\alpha$ and $\beta$ cytoplasmic domains to swing apart (Burridge and Chrzanowska-Wodnicka 1996; Tozer, Hughes et al. 1996). From these data, it was proposed that interaction of the cytoplasmic domains of integrin heterodimers by salt bridges maintains the molecule in a low-affinity state, and that disruption of this interaction results in ligand binding, an example of the phenomenon known as inside-out signaling. An implication of this phenomenon is that conformational changes in the cytoplasmic side of the integrins can be projected to the "business end" of the molecule in the extracellular space, thus affecting matrix binding affinity. A litany of proteins begins to assemble at 
nascent focal adhesions upon integrin clustering, some of which bind the cytoplasmic face of integrins, contributing to inside-out signaling. Additionally, certain of these proteins are also involved in signal transduction and/or linkage of the adhesion to the cytoskeleton. These include the adaptor proteins paxillin, vinculin, Crk, and Cas; the protein tyrosine kinases FAK and PYK2; and the actin linking proteins talin, $\alpha$-actinin, and filamin.

Paxillin is a $68 \mathrm{kD}$ protein constitutively present in focal adhesions. Like caveolin, paxillin was originally identified as a tyrosine-phosphorylated protein in $v$-Src-transformed fibroblasts by Glenney et al. (Glenney and Zokas 1989). Paxillin is recruited to focal adhesions via C-terminal LIM domains (Turner and Miller 1994). At the focal adhesion, paxillin is thought to interact with several adhesion molecules, including vinculin and focal adhesion kinase (FAK, see below), suggesting that paxillin may function as an adaptor protein involved in the assembly of supramolecular complexes that constitute adhesion architecture (Turner, Glenney et al. 1990; Hildebrand, Schaller et al. 1995). Moreover, tyrosine-phosphorylation of paxillin may influence adhesion signaling via recruitment of the protein Crk (Birge, Fajardo et al. 1993; Schaller and Parsons 1995).

Vinculin is a $116 \mathrm{kD}$ protein described by Pardo et al. in cardiomyocyte costameres, which function analagously to focal adhesions in nonmuscle cells (Craig and Pardo 1983; Pardo, Siliciano et al. 1983). Vinculin was later identified in focal adhesions, where it binds talin, $\alpha$-actinin, paxillin, and actin (Mangeat and Burridge 1984; Johnson and Craig 1994; Kroemker, Rudiger et al. 1994). Like 
paxillin, vinculin may contribute as an adaptor protein in adhesion architecture. Vinculin exists in an inactive conformation by an intramolecular "head-tail" interaction that precludes the protein from binding other molecules. Interestingly, binding of certain acidic phospholipids to vinculin disrupts the head-tail interaction; such binding may contribute to vinculin and focal adhesion dynamics in vivo (Fukami, Endo et al. 1994).

The docking protein $\mathrm{p} 130^{\mathrm{CAS}}$ (Cas) is a resident focal adhesion protein that interacts with FAK and recruits Crk to adhesions upon Src-dependent tyrosine phosphorylation (Mayer, Hamaguchi et al. 1988; Kanner, Reynolds et al. 1990). Cas-Crk interaction is thought to mediate downstream signaling through the proteins C3G and DOCK1 (Knudsen, Feller et al. 1994; Hasegawa, Kiyokawa et al. 1996), and seems to be important in Rac and Cdc42 activation, leading to actin nucleation and polymerization at the leading edge (see later).

Focal adhesion kinase (FAK) is a resident adhesion protein involved in scaffolding at and signal transduction from the adhesion (Aplin, Howe et al. 1998). Upon integrin clustering, FAK is recruited to nascent adhesions, whereupon it is rapidly autophosphorylated on tyrosine residue 397. Tyrosine-phosphorylated FAK is known to interact with several proteins, namely Src (Schaller and Parsons 1994), GRB2 (Schlaepfer and Hunter 1996), and PI3 Kinase (Chen and Guan 1994). Although several studies indicate that FAK is critically involved in adhesion turnover and motility, a recent report analyzing conditional FAK knockout in the endothelium indicated a primary defect in cell survival and lamellipodial formation, with no apparent decrease in migration (Braren, Hu et al. 
2006). Proline-rich tyrosine kinase 2 (PYK2) is another autophosphorylated kinase in focal adhesions, with a high structural homology and overlapping function with FAK, but with a more limited tissue distribution (Avraham, London et al. 1995). Compensation by PYK2 in cells derived from FAK null embryos has been hypothesized, perhaps weakening the phenotype and confounding the interpretation of FAK function (Aplin, Howe et al. 1998).

Several proteins directly link integrins to the actin cytoskeleton. Talin is a $270 \mathrm{kD}$ protein that exists a antiparallel-arranged homodimer. A FERM domain at the $\mathrm{N}$-terminus mediates binding to integrins, acidic phospholipids, and FAK (Brakebusch and Fassler 2003). The rod-like C-terminus contains a major actin binding site (Hemmings, Rees et al. 1996). Talin interaction with the cytoplasmic domain of integrins has been reported to disrupt the salt bridges between $\alpha$ and $\beta$ subunits, resulting in increased integrin affinity and ECM binding through inside-out signaling ((Vinogradova, Velyvis et al. 2002; Garcia-Alvarez, de Pereda et al. 2003), see above). Drosophila lacking talin still display integrinECM interaction, but clustering and association with the actin cytoskeleton is abrogated (Brown, Gregory et al. 2002).

a-actinin, well-known as an actin-bundling protein, also directly connects F-actin to clustered integrins (Burridge and Chrzanowska-Wodnicka 1996). Like talin, $\alpha$-actinin is arranged as an anti-parallel homodimer whose $\mathrm{N}$-terminus is responsible for actin binding (Noegel, Witke et al. 1987). Two non-muscle isoforms exist, of which the ubiquitously-expressed $\alpha$-actinin-1 is constitutively present in focal adhesions (Pavalko, Otey et al. 1991; Brakebusch and Fassler 
2003). An elegant study by Rajfur et al. demonstrated that a-actinin was essential for linkage of the cytoskeleton with integrins by chromophore-assisted laser inactivation of EGFP-a-actinin (Rajfur, Roy et al. 2002).

Filamin is also an actin-binding protein that may serve to link the actin cytoskeleton with integrins. Filamin exists as a homodimer mutually associated at the C-termini. Interaction with actin occurs at the $\mathrm{N}$-terminus, while integrins have been found to associate with the C-terminal rod domain (Aplin, Howe et al. 1998). Filamin linkage of integrins with the actin cytoskeleton has been suggested to play a role in cell spreading and migration (Sharma, Ezzell et al. 1995).

The number of resident focal adhesion proteins has been estimated in the range of 50 (Zamir and Geiger 2001). Moreover, the resolution of fluorescent microscopy, commonly used in the study of focal adhesions, is not sufficient to analyze position of adhesion proteins relative to one another within an adhesion; atomic force microscopy has potential to elucidate adhesion architecture, although this application has only very recently been explored (Franz and Muller 2005). Thus, large deficits in the understanding of adhesion architecture presently exist. In spite of this, much progress has been made in unraveling the dynamics of focal adhesions through study of moving cells.

Cell motility is an indispensable biological process, utilized in myriad ways beginning upon (indeed before) conception of an organism, continuing with development, and persisting in both the physiology and pathology of an organism throughout its life. Such migration is thought to contribute to disease 
development or progression in certain pathologies-for instance, the inflammatory response in autoimmune disease, and cancer cell invasion and metastasis-driving interest in identifying the molecular basis of cell motility. Moreover, insight gained by the study of cytoskeletal and adhesion dynamics during cell movement in vitro may not only be relevant to cell migration in vivo, but also any biological process in which cytoskeletal rearrangement occurs, for example, the remodeling response of the vasculature to an acute (change in shear rate) or a chronic stimulus (growth and development).

Cells migrating on a planar surface develop a characteristic front-rear asymmetry with coordinate functions (Horwitz and Parsons 1999). Specifically, in the lamellipodia, a large, sheet-like protuberance situated at the front of motile cells, F-actin polymerization against the plasma membrane and nascent adhesion formation drive membrane protrusion and attachment at the leading edge, whereas a concerted contraction of the actin cytoskeleton and detachment at the cell rear control cell body movement in the direction of lamellipodial protrusion.

Though focal adhesions act to fix the cell to the extracellular matrix, the nature of this interaction turns out to be highly dynamic, such that cells may readily spread and move in relation to their substratum. The study of adhesions in migrating cells has revealed a spatial disparity in their nature, providing clues about adhesion turnover during motility. Leading edge adhesions decorate the perimeter of the lamellipodia. These adhesions appear as small puncta with low integrin densities (Ballestrem, Hinz et al. 2001). Current evidence indicates that 
these nascent leading edge adhesions, known as focal complexes, are closely coupled with actin nucleation and protrusion that drive extension of the lamellipodia in the forward direction (Lee and Jacobson 1997; Webb, Parsons et al. 2002).

Focal complexes remain stationary with respect to the substrate until they disperse due to lamellipodial collapse or mature into larger focal adhesions (Ballestrem, Hinz et al. 2001). Coupling of focal complex formation to cortical actin polymerization is regulated by the small guanosine triphosphatases (GTPases) Rac and Cdc42, and occurs under a low tension environment (Horwitz and Parsons 1999; Ballestrem, Hinz et al. 2001). With an increase in cytoskeletal tension, as occurs with activation of Rho GTPase, traction on the focal complexes increases, inducing maturation of focal complexes into larger, more integrin-dense adhesions (Burridge and Chrzanowska-Wodnicka 1996; Pelham and Wang 1999; Ballestrem, Hinz et al. 2001; Beningo, Dembo et al. 2001). These mature leading edge adhesions persist more interiorly in the lamellipodia where they may serve as foci of traction for propulsion of the actin cytoskeleton (Galbraith and Sheetz 1997; Lee and Jacobson 1997; Palecek, Huttenlocher et al. 1998; Beningo, Dembo et al. 2001). These mature lamellipodial adhesions ultimately disassemble by a poorly understood process that optimally requires FAK, Src, Cas, paxillin, MLCK, and, surprisingly, the MAP kinase ERK (Webb, Donais et al. 2004).

Focal adhesion formation at the cell rear during migration is not as wellcharacterized as at the leading edge, although recent studies have disclaimed 
the notion that adhesions originating at the front of the cell persist to become trailing edge adhesions as the cell body moves forward (Ballestrem, Hinz et al. 2001; Rid, Schiefermeier et al. 2005). Rid et al. have demonstrated that trailing adhesions are formed de novo, either as focal complexes that arise from shortlived lamellipodia that form at the cell rear or underneath pre-existing actin stress fibers proximal to their trailing edge (Rid, Schiefermeier et al. 2005). Trailing focal adhesions, in contrast to focal complexes, are large and integrin-dense, under high tension due to association with actin stress fibers, and move in relation to the substratum (Ballestrem, Hinz et al. 2001). The movement of focal adhesions, termed contact plasticity, along with adhesion disassembly, result in tail retraction during migration.

Molecular events responsible for adhesion disassembly at the rear are poorly understood. Recent studies have implicated microtubule recruitment to adhesions sites as one possible mechanism (Kaverina, Krylyshkina et al. 1999; Ballestrem, Hinz et al. 2001; Ezratty, Partridge et al. 2005). In these studies, recruitment of microtubule plus ends to focal adhesions is apparent, but arrival at the adhesions does not necessarily trigger disassembly. The authors suggest that microtubule targeting might deliver an unknown "relaxing factor", itself responsible for adhesion breakdown and trafficked on microtubules. Other studies have implicated calpain-dependent proteolysis in adhesion turnover, as several focal adhesion proteins are known substrates for the protease (Palecek, Huttenlocher et al. 1998; Satish, Blair et al. 2005). 
Several studies have implicated caveolin-1 in focal adhesion dynamics and signaling, although a straightforward role for the molecule has not emerged. Wary et al. reported that caveolin-1 serves as a membrane adaptor to link the integrin $\alpha$ subunit to the tyrosine kinase Fyn, which subsequently binds Shc; and that these events are critical in linking integrins to the Ras-ERK pathway (Wary, Mariotti et al. 1998). Importantly, the authors report that caveolin-1 did not codistribute with focal adhesions, but with poorly-defined "extracellular matrix contacts", although these data were not included in the report. Wei et al. independently reported that anti-sense mediated depletion of caveolin-1 resulted in the loss of focal adhesion sites, ligand-induced focal adhesion kinase phosphorylation, and cell adhesion (Wei, Yang et al. 1999). Here also, the authors fail to show that caveolin-1 associates with focal adhesions, although immunostaining reveal partial colocalization of caveolin-1 and integrin $\beta 1$ in arrays over the cell body. Furthermore, the findings that fibronectin-induced FAK phosphorylation and cell adhesion were compromised with caveolin-1 suppression are not consistent with the viability of caveolin-1 mice, as well as the presence of focal adhesions, cell spreading, and normal FAK autophosphorylation in CAV-1-/- MEFs (del Pozo, Balasubramanian et al. 2005). This discrepancy might represent a cell-type specific requirement of caveolin-1 for integrin-mediated adhesion, or perhaps a differential response between acute and chronic downregulation of caveolin-1; however, positive regulation of adhesion signaling through caveolin-1 may be the exception and not the rule. 
The role of caveolin-1 in cell motility, similar to adhesion dynamics, is not presently understood. Intriguingly, several reports have described the polarization of caveolin-1 to the rear of migrating cells in vitro (Isshiki, Ando et al. 2002; Parat, Anand-Apte et al. 2003; Beardsley, Fang et al. 2005). Anderson et al. first described the phenomenon in migrating bovine aortic endothelial cells (BAECs), and noted a correlation between polarization of caveolin-1 and sites of calcium wave initiation. Moreover, exposure of endothelial cells to laminar shear stress results in an equivalent polarization of caveolin-1 in the region of the cell nearest the direction of media flow, designated the "upstream edge" (Isshiki, Ando et al. 2002).

Parat et al. reported the dependence of caveolin polarization on the mode of migration, i.e. planar- versus trans-migrating cells. Rear polarization of caveolin-1 in conjunction with caveolae was observed in planar migrating cells, similar to the earlier report by Anderson (Parat, Anand-Apte et al. 2003). In BAECs migrating through a transwell pore, however, caveolin-1 was distributed towards the front of the cell, while caveolae remained concentrated at the cell rear. The phosphorylation of tyrosine residue 14 was shown to be required for frontward polarization in transwell migration, but not for rear polarization during planar movement. The existence of caveolae devoid of caveolin is problematic, as CAV-1-/- mice are devoid of morphological caveolae, indicating that caveolin1 is indispensable for caveolae architecture (see above).

The authors concluded that phosphorylation is critical for frontwards polarization of caveolin-1 during transmigration, because expression of the 
unphosphorylatable caveolin-1 $\beta$-GFP fusion protein did not result in such polarization. This is also problematic, given the normally inordinately low ratio of phosphorylated to unphosphorylated caveolin-1 (del Pozo, Balasubramanian et al. 2005). The failure to detect front-polarized caveolin-1 $\beta$ suggests that every molecule of caveolin must be phosphorylated to polarize, as opposed to a fractional increase in phosphorylation triggering mass exodus of caveolin to the front, since endogenous, phosphorylatable caveolin was still present. The complete segregation of caveolin-1 and caveolae would thus necessitate $100 \%$ phosphorylation of the former, which is extremely unlikely given the low stoichiometry of caveolin phosphorylation. A plausible interpretation of the data would be the partial displacement of soluble caveolin from caveolae during migration through a transwell pore. Given the presence of caveolae at the cell rear during trans-migration, caveolin is necessarily present at the rear as well. The necessity of caveolin phosphorylation for caveolar egress would thus certainly be reasonably, albeit on a smaller scale than reported.

Besides the in vivo studies previously described, several lines of in vitro data indicate a positive role for caveolin-1 in vascular remodeling and angiogenesis, and by implication, focal adhesion dynamics and cell migration. Several studies demonstrated that cholesterol treatment or down-regulation of caveolin by antisense oligonucleotides or adenovirus reduces capillary-like tube formation by human microvascular endothelial cells or human umbilical vein endothelial cells (HUVECs) on matrigel (Griffoni, Spisni et al. 2000; Liu, Wang et al. 2002; Galvez, Matias-Roman et al. 2004). Conversely, expression of the 
caveolin scaffolding domain increases tube formation in microvascular cells. Furthermore, down-regulation of caveolin-1 by small interfering RNA decreases the motility of HUVECs (Beardsley, Fang et al. 2005), and inhibits blood vessel development in chick embryo chorioallantoic membrane (Griffoni, Spisni et al. 2000).

Thus, current evidence suggests a role for caveolin-1 in cell motility and cytoskeletal remodeling. The interaction between caveolin-1, focal adhesions, and the actin cytoskeleton remains poorly understood, however. The aim of the current project is to analyze how caveolin-1 functions during endothelial cell migration. Specifically, we attempt to address the significance of caveolin polarization to the cell rear, especially in the context of the cytoskeletal and focal adhesion dynamics at the trailing edge. 


\section{Study 1}

Loss of Caveolin-1 Polarity affects Endothelial Cell Polarization and Directional Movement 


\section{ABSTRACT}

The ability of a cell to move requires the asymmetrical organization of cellular activities. To investigate polarized cellular activity in moving endothelial cells, human endothelial cells were incubated in a Dunn chamber to allow migration towards VEGF. Immunofluorescent staining with a specific antibody against caveolin-1 revealed that caveolin-1 was concentrated at the rear of moving cells. Similarly, monolayer scraping to induce random cell walk resulted in relocation of caveolin-1 to the cell rear. These results indicate that posterior polarization of caveolin-1 is a common feature both for chemotaxis and chemokinesis. Dual immunofluorescent labeling showed that, during cell spreading, caveolin-1 was compacted in the cell center and excluded from nascent focal contacts along the circular lamellipodium revealed by integrin $\beta 1$ and FAK staining. When cells were migrating, integrin $\beta 1$ and FAK appeared at polarized lamellipodia, whereas caveolin-1 was found at the posterior of moving cells. Notably, wherever caveolin-1 was polarized, there was a conspicuous absence of lamellipod protrusion. Transmission electron microscopy showed that caveolae, like their marker caveolin-1, were located at the cell center during cell spreading or at the cell rear during cell migration. In contrast to its unphosphorylated form, tyrosine-phosphorylated caveolin-1, upon fibronectin stimulation, was associated with the focal complex molecule phospho-paxillin along the lamellipodia of moving cells. Thus, unphosphorylated and phosphorylated caveolin-1 were located at opposite poles during cell migration. Importantly, loss of caveolin-1 polarity by targeted down-regulation of the protein prevented cell polarization and directional movement. Our present results 
suggest a potential role of caveolin polarity in lamellipod extension and cell migration. 


\section{INTRODUCTION}

Endothelial cell migration is a key step towards angiogenesis, a process that is required in a variety of physiological and pathological conditions, such as embryonic development, wound healing, tissue regeneration and tumor growth and metastasis. A clear understanding of how endothelial cells sense chemoattractants, organize signaling asymmetry and make a directional movement is of pivotal importance in the biology of normal cells as well as tumor angiogenesis. Like other types of crawling cells, migrating endothelial cell acquires a series of spatially polarized features. The front of a migrating cell generates protrusive force associated with lamellipod or filopod protrusion coupled with the development of new cell adhesions to the extracellular substrates. Cell contractility is required to allow the retraction of the body and rear of the cell. Apart from the surface features, such as lamellipodia and microspikes, relatively little is known about "directional sensing" machinery that orients locomotion machinery in endothelial cells.

Caveolae (also termed plasmalemmal vesicles) are specialized microdomains on the plasma membrane with a size of 50-100 nm (Anderson 1998). In addition to transcytosis and endocytosis, a good body of evidence has shown that caveolae compartmentalize and integrate signaling events at the cell surface (Anderson 1993; Lisanti, Scherer et al. 1994; Liu, Oh et al. 1997). A variety of protein and lipid signaling molecules involved in VEGF receptor and integrinmediated signaling are concentrated in caveolae. These include VEGF-R2 (KDR), non-receptor tyrosine kinases (NRTK) such as Src, Yes and Fyn, PI 3-kinase, 
Rac1, Cdc42 and RhoA, and phosphatidylinositol (Lisanti, Scherer et al. 1994; Pike and Casey 1996; Liu, Oh et al. 1997; Gingras, Gauthier et al. 1998; Kawamura, Miyamoto et al. 2003; Labrecque, Royal et al. 2003). These observations suggest a potential role of caveolae in mediating signal transduction involved in cell migration.

The major structural proteins of caveolae are the caveolins. Four caveolins encoded by three different genes have been identified. Endothelial cells abundantly express caveolin-1 and -2 , but not caveolin-3 which is musclespecific (Song, Scherer et al. 1996). Caveolin-1 interacts with a number of signaling proteins including Src family kinases, G $\alpha$ subunits, H-Ras, PKC, eNOS, PI 3-kinase, integrins and EGF-R (Okamoto, Schlegel et al. 1998; Wary, Mariott et al. 1998; Wei, Yang et al. 1999; Zundel, Swiersz et al. 2000). In general, interaction between caveolin via the caveolin-scaffolding domain (CSD) and signaling proteins leads to inactivation of the target proteins (Okamoto, Schlegel et al. 1998). Thus, caveolin may function as an endogenous negative regulator of many signaling molecules. Given this view, one would predict that downregulation of caveolins may lead to an increase in basal activity of signaling pathways and subsequent cellular activity such as cell motility. In accordance with this, an attractive hypothesis would be that mere translocation of caveolin (i.e. caveolin polarization) without a substantial change in the expression level would reinforce an inhibitory effect on one part of the cell, i.e., the cell rear, but release its inhibitory activity on the other side, i.e., the leading edge. This hypothesis is supported by recent studies showing caveolae and caveolin-1 
asymmetry during cell migration (Okada, Tomaszewski et al. 1995; Folkman 2002; Isshiki, Ando et al. 2002; Parat, Anand-Apte et al. 2003). However, whether caveolin-1 polarity affects endothelial cell lamellipod protrusion and migration is not known.

In the present study, we demonstrate that caveolin-1 and caveolae were excluded from the leading edge and directed toward the rear of migrating cells. Interestingly, a subpopulation of caveolin-1 that was phosphorylated on tyrosine 14 specifically moved to focal adhesions at the leading edge of migrating cells. Importantly, loss of caveolin-1 polarity by targeted knockdown of the protein prevented endothelial cell polarization and impeded cell directional movement. 


\section{EXPERIMENTAL PROCEDURES}

Materials: Reagents and other supplies were obtained from the following commercial sources: antibodies against caveolin-1, phospho-caveolin-1, and FAK from BD Biosciences (San Diego, CA). Phalloidin-TRITC and fibronectin were purchased from Sigma Chemicals (St. Louis, MO). Antibodies against integrin $\beta 1$, GFP, and c-Myc were purchased from Santa Cruz Biotechnology (Santa Cruz, CA). Goat serum, FITC and Rhodamine Red-X conjugated secondary antibodies were obtained from Jackson Immuno-Research Laboratories (West Grove, PA). Antibodies against paxillin, phospho-paxillin (pTyr-31) and phospho-FAK (pTyr-397) were obtained from Biosource International (Camarillo, CA). Slow-fade reagent was purchased from Molecular Probes (Eugene, OR). The bicinchoninic acid (BCA) protein assay kit was purchased from Pierce (Rockford, IL).

Cell culture. Primary cultured endothelial cells from human umbilical veins (HUVECs) were obtained as described previously (Ashton, Yokota et al. 1999) from consenting healthy, term patients according to institutional guidelines. HUVECs were grown in Medium 199 supplemented with 5\% heat-inactivated human serum, $20 \%$ heat-inactivated new born calf serum and endothelial cell growth supplement.

Construction of siRNA. The sequence (AAGAGCTTCCTGATTGAGATT) was selected as the targeting region of caveolin-1, which corresponds to nucleotides 403-423 of the coding region of human caveolin-1 (GenBank accession number BC009685). Caveolin-1 siRNA primers (5'-AATCTCAATCAGGAAGCTCTT-3' 
and 5'-GAGCTTCCTGATTGAGATTTT-3') were used for synthesis of double stranded RNA (dsRNA) by Qiagen (Valencia, CA). Control siRNA was also obtained from Qiagen. The target sequence of the control siRNA was the DNA sequence AATTCTCCGAACGTGTCACGT, which contains a 16 base overlap with Thermotoga maritimia (GenBank accession number AE001709) section 21 of 136 of the complete genome, with no other BLAST matches. The level of caveolin-1 expression was determined by Western blot analysis and immunocytochemistry using specific antibody against caveolin-1. The specific down-regulation of caveolin-1 expression by caveolin-1 siRNA was assessed using control siRNA.

Transfection of endothelial cells with siRNA. Endothelial cells were seeded at a density of $6 \times 10^{4}$ cells/well in a 6 -well plate 24 hours prior to transfection. For each transfection, $1.0 \mu \mathrm{g}$ of dsRNA were diluted into $200 \mu \mathrm{l}$ of serum-free medium in a tube. In another tube, $6 \mu$ l of Oligofectamine (Invitrogen) were mixed with $54 \mu \mathrm{l}$ of serum-free medium. The two solutions were combined, mixed gently, and incubated for $30 \mathrm{~min}$ at room temperature followed by the addition of $400 \mu \mathrm{l}$ of serum-free medium. Cells were washed once with serum-free medium, and incubated with dsRNA mixtures or Oligofectamine-medium (mock transfection) for $4 \mathrm{~h}$ at $37^{\circ} \mathrm{C}$ in a humidified $\mathrm{CO}_{2}$ incubator. At the end of incubation, $2 \mathrm{ml}$ of growth medium were added to each well and cells were incubated for 20 hours. The medium was replaced with growth medium $24 \mathrm{~h}$ after transfection. Fortyeight hours post-transfection (which resulted in approximately $80 \%$ reduction in caveolin-1 level), the cells were used for migration assay. 
Scratch motility assay. Confluent endothelial cells were wounded by scraping of the monolayer with a $200 \mu \mathrm{l}$ pipette tip (Tang, Morgan et al. 1997). Cultures were washed once with PBS and incubated with growth medium. Cells were incubated at $37^{\circ} \mathrm{C}$ for 10 hours to allow migration towards the gap and then fixed, permeabilized and stained for caveolin-1.

Dunn chamber assay. Endothelial cells were seeded on fibronectin-coated coverslips and starved for 24 hours prior to assay. To set up gradient experiments, both concentric wells of the chamber were filled with starvation medium (medium with $0.5 \%$ FBS), and a coverslip seeded with cells was inverted onto the chamber in an offset position leaving a narrow slit at one edge for refilling the outer well. The coverslip was sealed in place using hot wax mixture around all the edges except for the filling slit. The medium of the outer well was drained and replaced with medium containing $0.5 \%$ FBS and $10 \mathrm{ng} / \mathrm{ml}$ VEGF. The slit was then sealed with hot wax mixture. When the inner circular well of the chamber was filled with control medium and the outer annular well with medium containing chemoattractant, a radially directed linear diffusion gradient was established in the diffusion gap within 10-30 min, which had a half-life of 1030 hours (Zicha, Dunn et al. 1991). For control experiments in which cells were subjected to uniform concentrations of chemoattractant, both wells were filled with medium containing VEGF $(10 \mathrm{ng} / \mathrm{ml})$. At the end of each migration assay, the coverslip was carefully removed from the Dunn chamber, and the cells were processed for immunocytochemistry analysis. 
Immunocytochemistry. Endothelial cells were fixed with 2\% paraformaldehyde for 20 min, permeabilized with $1 \%$ Triton $X-100$, blocked with $5 \%$ goat serum, and stained with specific antibody against caveolin-1 or other signaling molecules. Bound primary antibodies were detected using fluorescein- or rhodamineconjugated secondary antibodies. The immunostained cells were mounted in the presence of Slow-Fade reagent. Immunostaining was visualized and photographed using a Zeiss LSM 510 confocal microscope.

Immunoblot analysis. Proteins of the subcellular fractions were solubilized with SDS-sample buffer containing $0.125 \mathrm{M}$ Tris-HCl (pH 6.8), 5\% (w/v) SDS, 2.5\% (v/v) $\beta$-mercaptoethanol, $5 \%$ glycerol in double distilled water. After boiling for 4 min, proteins were separated by SDS-PAGE $(5-15 \%$ gradient gels) and electrotransferred to a nitrocellulose membrane for immunoblotting using enhanced chemiluminescence (ECL).

Transmission electron microscopy. Endothelial cells were either sparsely seeded onto gelatin-coated plates for 1 hour or seeded near confluence, scratched multiply with a $200 \mu \mathrm{l}$ pipette tip, and incubated to allow cell migration. The cells were then fixed with $2.5 \%$ glutaraldehyde in $0.1 \mathrm{M}$ phosphate buffer and scraped into a microcentrifuge tube. The cells were pelleted and post-fixed with osmium tetroxide. After fixation, cells were infiltrated with a propylene oxide/epon mixture and stained with uranyl acetate and lead citrate. Thin sections of samples were examined under a JEOL 1220 transmission electron microscope. On randomly taken photographs, the number of caveolae at the leading edge and the cell 
center of spreading cells or at the leading edge and the cell posterior of migrating cells was determined. 


\section{RESULTS}

\section{Caveolin-1 is located at the rear of migrating cells}

Previous studies have shown that caveolin-1 displays polarization in migrating cells. For instance, caveolin-1 accumulated at the trailing edge of scratchinduced migrating BAECs or shear stress-stimulated BAECs (Isshiki, Ando et al. 2002; Parat, Anand-Apte et al. 2003). On the other hand, caveolin-1 was concentrated at the leading edge of FGF-stimulated migrating BAECs (Parat, Anand-Apte et al. 2003). To assess whether the discrepancy of caveolin-1 polarization resulted from chemotactic gradient, we employed two kinds of cell migration systems: chemokinesis to measure random walk using monolayer denudation (also termed scratch motility assay) and chemotaxis to measure directional movement using the Dunn chamber. Six-hours after scraping, endothelial cells began to migrate into the wound gap. The closure of wound gaps took about 24-hours. Immunofluorescent staining with a specific antibody against caveolin-1 showed that the majority of caveolin-1 was located at the rear of most moving cells (green arrowheads, b,c in Figure 1) opposite the lamellipodium of the leading edge (green arrows, Figure 1c). Similarly, when cells were exposed to a linear gradient of VEGF, caveolin-1 was concentrated at the rear or trailing edge of moving cells (green arrowheads, Figure 1d). These results indicate that caveolin-1 polarization at the cell posterior is a common feature of both directional and random movements. 


\section{Mutual exclusion between caveolin-1 and focal contacts at the leading edge}

The migration-activated posterior relocation of caveolin-1 is intriguing and suggests that when cells are stimulated to migrate, caveolin-1 (along with caveolae; see Figure 3) moves to the rear of the migrating cell as a mechanism to sequester it away from signaling proteins that direct cell motility at the leading edge. Here, we focused on nascent focal adhesions at the leading edge revealed by immunostaining with specific antibody against integrin $\beta 1$ and FAK. Human umbilical vein endothelial cells were seeded on fibronectin-coated coverslips. Within one-hour after seeding, most cells spread radially. As shown in Figure 2, caveolin-1 was localized compactly in the center of the spreading cells $(a, g$ in Figure 2), and was excluded from nascent focal contacts along the circular lamellipodium revealed by FAK or integrin $\beta 1$ staining ( $b, h$ in Figure 2). After incubation for several hours, focal contacts appeared in the polarized lamellipodia at the leading edge of migrating cells (e,k in Figure 2). Caveolin-1 signal was now relocated at the rear of most moving cells ( $d, j$ in Figure 2$)$. These data confirm that caveolin-1 moves to the opposite pole of a migrating cell, compared to FAK and $\beta 1$ integrin at the leading edge.

\section{Exclusion of caveolae from lamellipodia.}

Like many other terminally differentiated cells, such as adipocytes, fibroblasts and skeletal muscle cells, endothelial cells possess a large number of caveolae (Peters, Carley et al. 1985; Anderson 1998). Rapid-freeze deep-etch images show that caveolae have a striated coat and contain the integral 22-kDa 
membrane protein, caveolin (Rothberg, Heuser et al. 1992). The coat protein is a reliable marker for tracing caveolae trafficking in live cells (Mundy, Machleidt et al. 2002). Based on our results described above demonstrating that caveolin-1 was centrally concentrated in spreading cells, we predicted that caveolae may be centrally localized as well. To assess the location of caveolae, endothelial cells were seeded for one hour to allow spreading, fixed and processed for electron microscopy analysis. As shown in Figure 3, caveolae were localized abundantly at the cell center of spreading cells (arrowheads, $b$ in Figure 3A; Figure 3B). Few, if any, caveolae were found at the lamellipod protrusion (Figure 3B). When cells were migrating, caveolae, like their marker caveolin-1, were now concentrated at the cell rear (arrowheads, $d$ in Figure 3A; Figure 3C), opposite the lamellipodium. In contrast, few, if any, caveolae were found at the leading edge of migrating cells (e in Figure 3A; Figure 3C). Again, these results indicate that caveolae, along with caveolin-1, were excluded from leading edge of migrating cells.

\section{Phospho-caveolin-1 is co-localized with focal complex molecules at the leading edge of migrating cells}

Caveolin is one of the major v-Src substrates in Rous sarcoma virus-transformed chicken embryo fibroblasts (Glenney and Zokas 1989). Stimulation of A431 cells with EGF or NIH $3 \mathrm{~T} 3$ cells with hyperosmotic stress leads to phosphorylation of caveolin-1 on tyrosine 14 and the phosphorylation events are associated with focal adhesions (Lee, Volonte et al. 2000; Volonte, Galbiati et al. 2001). To assess whether phosphorylation of caveolin-1 occurred and where the phosphorylation took place during cell migration, endothelial cells were treated 
with fibronectin and dually stained with specific antibodies against phosphocaveolin-1 and either phospho-paxillin or caveolin-1. Dual immunolabeling revealed that phospho-caveolin-1 co-localized with the focal adhesion marker, phospho-paxillin, at the leading edge of migrating cells (a-c in Figure 4), indicating that caveolin-1 was associated with focal complexes along the lamellipodium concomitant with tyrosine 14 phosphorylation. In contrast, a significant population of caveolin-1 that was not immunoreactive with Tyr-14 phosphospecific antibodies localized to the rear of migrating cells (e,f in Figure 4). These data indicate that when tyrosine-phosphorylated, caveolin-1 associated with focal complex molecules at the leading edge of a migrating cell.

\section{Targeted knockdown of caveolin-1 impedes cell polarity}

Our results demonstrate that caveolin-1 was directed to the posterior of either directionally or randomly moving cells, suggesting that caveolin-1 may play an important role in cell movement. We reasoned that during migration, caveolin-1 together with caveolae moves to the rear of a migrating cell as a mechanism to sequester it away from signaling proteins that direct cell motility at the leading edge and that loss of caveolin-1 asymmetry by down-regulation of the protein would impede cell polarity and hence, cell migration. To test this hypothesis, we employed RNA interference to knock down caveolin-1 and examine its effect on cell polarity and migration. HUVECs were transfected with either caveolin-1 siRNA or control siRNA for forty-eight hours, and then seeded on fibronectin and incubated to allow migration. As shown in Figure 5A, transfection with caveolin-1 siRNA specifically knocked down caveolin-1 levels by more than $80 \%$. Notably, 
knockdown of caveolin-1 dramatically impeded the ability of endothelial cells to polarize, which instead maintained a near-circular lamellipodium ( $b$ in Figure $5 B$ ), while control siRNA- or mock-treated cells were able to polarize as normal ( $a, c$ in Figure 5B). A comparison of Cav-1 siRNA-treated with control siRNA- or mocktreated cells revealed a significant reversal of the ratio of circular to polarized cells in the Cav-1 siRNA group, with caveolin-1 knockdown inhibiting the polarization of endothelial cells (Figure $5 \mathrm{C}$ ).

\section{Knockdown of caveolin-1 inhibits endothelial cell directional movement.}

Given the nature of caveolin-1 as a scaffolding protein to organize and sequester signaling molecules, caveolin-1 may coordinate cellular activities between the leading edge and rear of a moving cell. In response to environmental stimulation, cells exclude caveolin-1 from the leading edge by an unknown mechanism and eliminate its inhibitory action on signaling molecules that are involved in lamellipod protrusion. At the same time, caveolin-1 concentrates and reinforces its inhibitory action at the posterior of a polarized cell. Hence, a cell may sense and move directionally by exclusion of caveolin-1 from the leading edge. In accordance with this, loss of caveolin polarity would inhibit cell directional movement. To test this hypothesis, HUVECs were transfected with caveolin-1 siRNA and subjected to chemotactic response to serum using a well characterized microchemotaxis chamber system (Segall, Tyerech et al. 1996). As shown in Figure 6, loss of caveolin-1 polarity by knockdown of the protein dramatically reduced the number of migrating endothelial cells by more than 
three fold over mock or control siRNA-treated cells. Thus, our results indicate that loss of caveolin-1 asymmetry impeded endothelial cell directional movement. 


\section{DISCUSSION}

We have demonstrated that caveolin and caveolae polarized regardless of VEGF-induced directional movement or monolayer scraping-induced random walk. Polarization was found at the rear of moving cells in both models. Notably, during cell spreading or migration, extension of the leading edge and protrusion of lamellipodia were not observed in the area with higher caveolin-1 signal. Although caveolin has an intimate relationship with the cytoskeleton, little is known about the function of the protein in cell migration. In the present study, we have shown that loss of caveolin polarity by knockdown of the protein dramatically inhibited cell polarization and impeded cell directional movement. Our results indicate an essential role of caveolin polarity in lamellipod protrusion and orienting directional movement in endothelial cells. Figure 7 depicts a model of cell polarization in which caveolin-1 is located at the rear of a moving cell where it prevents lamellipod protrusion. Upon phosphorylation at tyrosine 14, caveolin-1 is released from caveolae and associated with focal adhesion sites at the leading edge where it may mediate the recruitment of Csk and affect the formation of focal adhesions (Cao, Sanguinetti et al. 2004).

Our present results are consistent with previous reports demonstrating caveolin and caveolae polarization in bovine aortic ECs in response to shear stress (Isshiki, Ando et al. 2002), monolayer scraping or FGF stimulation (Parat, Anand-Apte et al. 2003). Our result of growth factor-induced caveolin relocation at the cell posterior stands in contrast to a recent report that caveolin is located to the leading edge of FGF-stimulated transmigrating BAECs (Parat, Anand-Apte et 
al. 2003). Interestingly, the leading edge accumulation of caveolin-1 requires phosphorylation of the protein at tyrosine 14 , which is consistent with our finding that tyrosine-phosphorylated endogenous caveolin-1 associated with focal adhesion molecules at the leading edge (see Figure 4). This study did not determine if the polarized endogenous caveolin-1 is tyrosine-phosphorylated, therefore, it is not clear whether they were observing forward relocation of nonphosphorylated or phosphorylated caveolin-1. Manes and colleagues (Manes, Mira et al. 1999) showed recently the relocation of lipid rafts to the leading edge of insulin-like growth factor-I stimulated MCF-7 adenocarcinoma cells. Since the study did not examine the location of caveolin-1, a marker of caveolae, it is unclear that the relocation of lipid rafts represents caveolae or non-caveolae rafts. Thus, caveolin and caveolae relocation depends on the modes of cell migration and is cell-type specific. Nonetheless, the observation that different stimulations lead to caveolin and caveolae relocation suggests that it is a general mechanism for cells to spatially organize subcellular activities that direct cell motility.

The majority of caveolin-1 is found at the cell surface and associated with caveolae (Rothberg, Heuser et al. 1992; Das, Lewis et al. 1999). Some Golgiassociated caveolin-1 is in transit from its site of synthesis in the ER to the cell surface (Monier, Parton et al. 1995). The recycling of surface caveolin-1 through the Golgi apparatus involves the directional movement of the molecule from caveolae to the lumen of the ER and onto the Golgi apparatus (Smart, Ying et al. 1994; Conrad, Smart et al. 1995). One possible mechanism that directs caveolae and caveolin-1 polarization is that recycling caveolar vesicles accumulate at the 
cell rear during migration. Another possibility is that caveolin-1 and caveolae accumulate at the cell posterior as a result of differential movement of other organelles. The experimental observation that cell polarization signals are upstream and independent of those triggering cell motility suggests that asymmetric caveolin redistribution is not the consequence of cell movement (Manes, Mira et al. 2000; Meili and Firtel 2003).

Caveolin-1 was first identified as a major tyrosine phosphorylated protein in vSrc transformed chicken embryo fibroblasts (Glenney and Zokas 1989). Microsequencing of Src-phosphorylated caveolin-1 revealed that phosphorylation occurs within the extreme N-terminal region of the full-length of caveolin-1 ( $\mathrm{Li}$, Seitz et al. 1996). Site-directed mutagenesis showed that tyrosine 14 is the principal substrate for Src kinase (Li, Seitz et al. 1996). Recent studies have shown that caveolin-1 undergoes phosphorylation at tyrosine 14 in response to a number of stimulations such as insulin, EGF, and osmotic stress. Tyrosinephosphorylated caveolin-1 provides a docking site recruiting SH2-domain containing proteins, such as Grb7 and Csk, and augments EGF-stimulated cell migration (Lee, Volonte et al. 2000; Cao, Sanguinetti et al. 2004). Since Grb7 contains an $\mathrm{SH} 2$ domain and a PIR (phosphotyrosine-interacting region) (KasusJacobi, Bereziat et al. 2000), it may function as bridge linking phosphorylated caveolin-1 to other tyrosine-phosphorylated proteins, such as FAK (Han and Guan 1999). In the present studies, we have observed a rapid phosphorylation of caveolin-1 at tyrosine 14 upon fibronectin stimulation. Importantly, unlike the unphosphorylated caveolin-1, tyrosine-phosphorylated caveolin-1 was co- 
localized with focal complex molecules at the leading edge of migrating cells. The discovery that tyrosine-phosphorylated caveolin-1 and unphosphorylated caveolin-1 polarize at two opposing poles of moving cells is intriguing, although the mechanism underlying this is unclear. Therefore, during cell migration, phosphorylated caveolin-1 located at focal complexes along the leading edge might couple integrin to non-receptor tyrosine kinases such as Src, Fyn and FAK, whereas unphosphorylated inhibitory caveolin-1 is relocated to the cell rear.

Our present results demonstrate that loss of caveolin polarity impeded endothelial cell polarity and directional movement and suggest that caveolin may play an important role in angiogenesis. This idea is supported by a variety of studies showing that caveolin-1 affects capillary formation. We and others have shown recently that antisense-mediated down-regulation of caveolin-1 inhibits capillary tubule formation (Griffoni, Spisni et al. 2000; Liu, Wang et al. 2002). Upregulation of caveolin-1 in microvascular endothelial cells enhances capillary tubule formation via caveolin-1 scaffolding domain (Liu, Wang et al. 2002). The importance of caveolin-1 in angiogenesis is further emphasized by a recent study demonstrating a reduced infiltration of blood vessels into FGF-supplemented Matrigel plugs in caveolin-1 knockout mice (Woodman, Ashton et al. 2003). In the same mice, tumor weight, volume and blood vessel density are reduced due to lack of caveolin-1 and caveolae (Woodman, Ashton et al. 2003). 

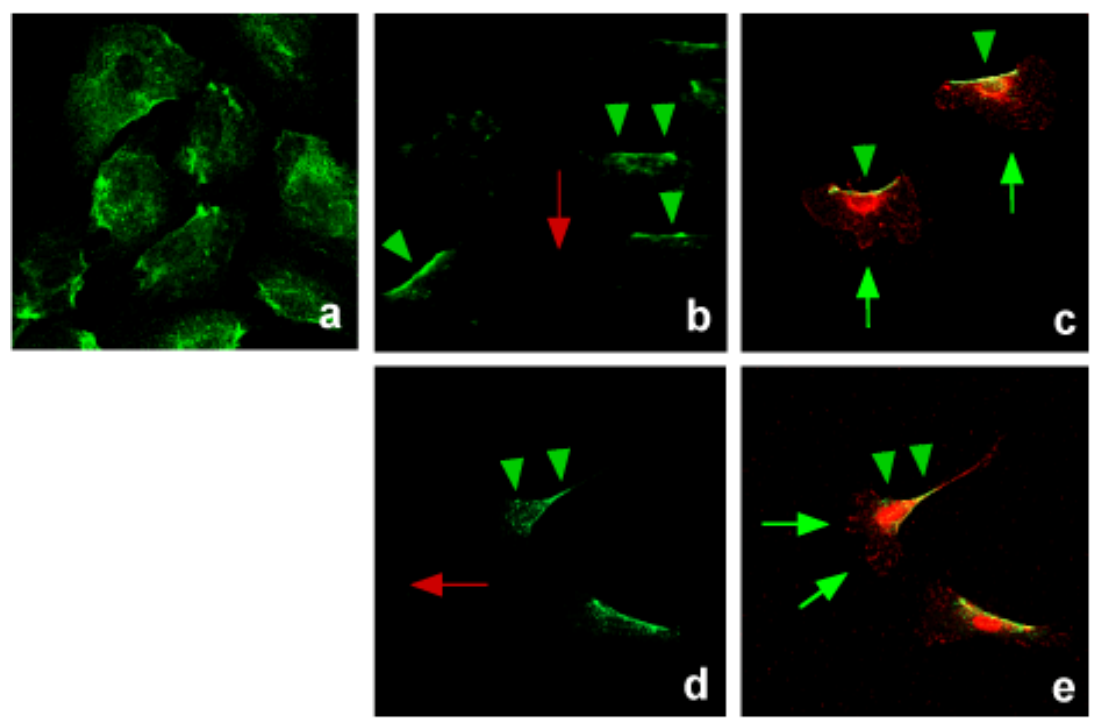

Figure 1. Caveolin-1 polarization during cell migration. Confluent HUVECs were either cultured on coverslips (a) or wounded by scraping the monolayer with a pipette tip $(b)$, incubated for 10 hours to allow migration towards the gap (red arrow in $b$ ) and then fixed and immuno-stained with antibody for caveolin-1 $(a, b)$ or dually stained with caveolin-1 (FITC) and integrin $\beta 1$ (Rhodamine Red-X) (c) to reveal the leading edge of a migrating cell. Note that when cells migrate toward the wound gap, caveolin-1 was relocated to the rear of moving cells (green arrowheads in $b, c$ ), opposite the lamellipodia (green arrows in $c$ ). Dunn chamber assay $(d, e)$. HUVECs were seeded on coverslips pre-coated with fibronectin and incubated at $37^{\circ} \mathrm{C}$ to allow migration toward VEGF (red arrow in $d$ ) until they appeared in the bridge and outer well of the chamber (see Materials and Methods). At that time the coverslips were removed from the chamber and the cells were fixed and subjected to dually immunofluorescent staining with specific antibody for caveolin-1 (FITC, d) and integrin $\beta 1$ (Rhodamine Red-X, e). Note that caveolin-1 signal was again localized at the rear and trailing edge of moving cells (green arrowheads in $d, e$ ), opposite the leading edge (green arrows in $e$ ). 

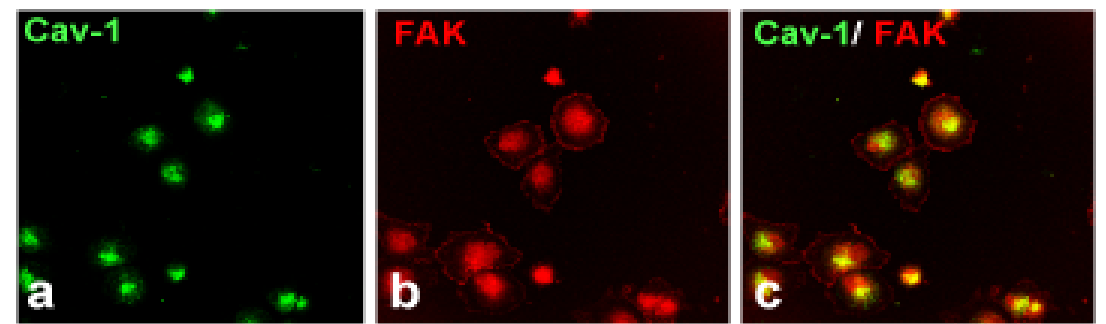

\section{Spreading}
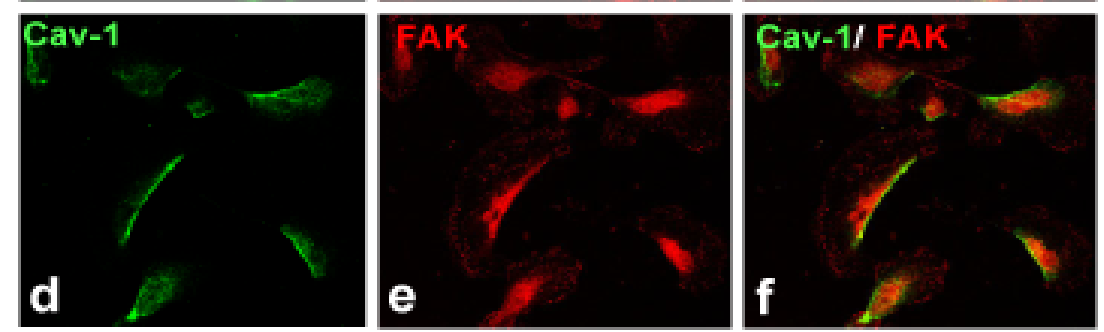

Migration
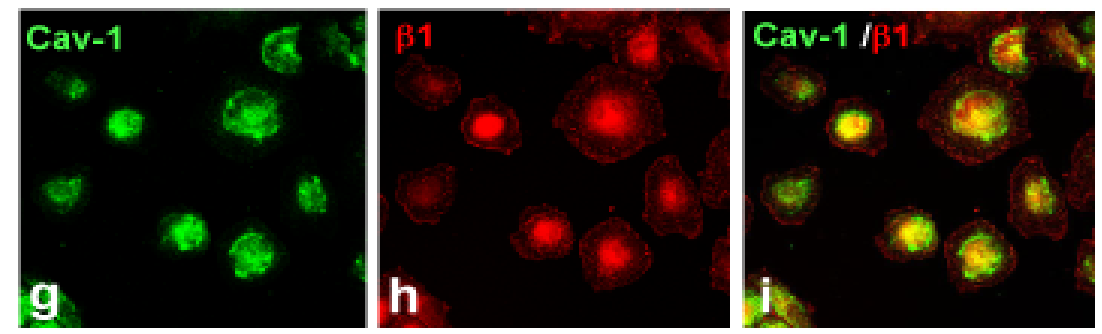

Spreading
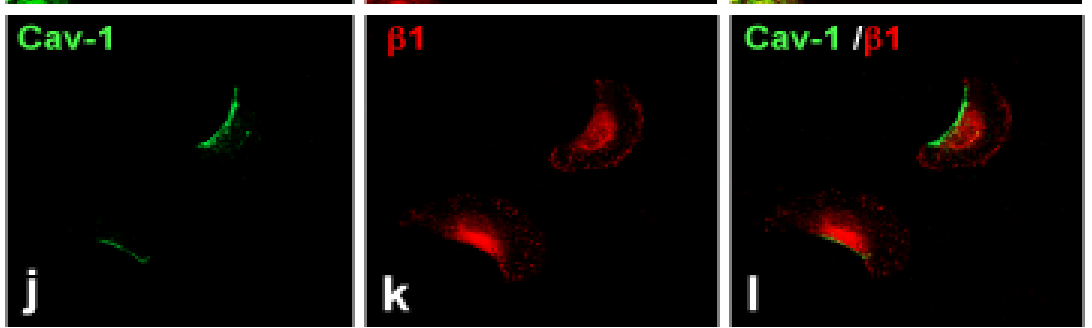

\section{Migration}

Figure 2. Mutual exclusion between caveolin-1 and focal contacts at the leading edge. HUVECs were seeded on fibronectin-coated coverslips and incubated at $37^{\circ} \mathrm{C}$ either for cell spreading assay or cell migration assay. After incubation, cells were fixed, permeabilized and subjected to dual immunofluorescent staining with caveolin-1 $(a, d, g, j)$ and either FAK $(b, e)$ or integrin $\beta 1(h, k)$. Composite images $(c, f, i, l)$ resulting from the superimposition of caveolin-1 signal and FAK or integrin $\beta 1$ are shown. Note that during cell spreading, caveolin-1 was excluded from nascent focal contacts revealed by FAK and integrin $\beta 1$ staining along the leading edge and located compactly in the cell center. When cells were migrating, focal contacts were now localized to one or sometimes two polarized lamellipodia, whereas caveolin-1 appeared at the body and rear of moving cells. 
A

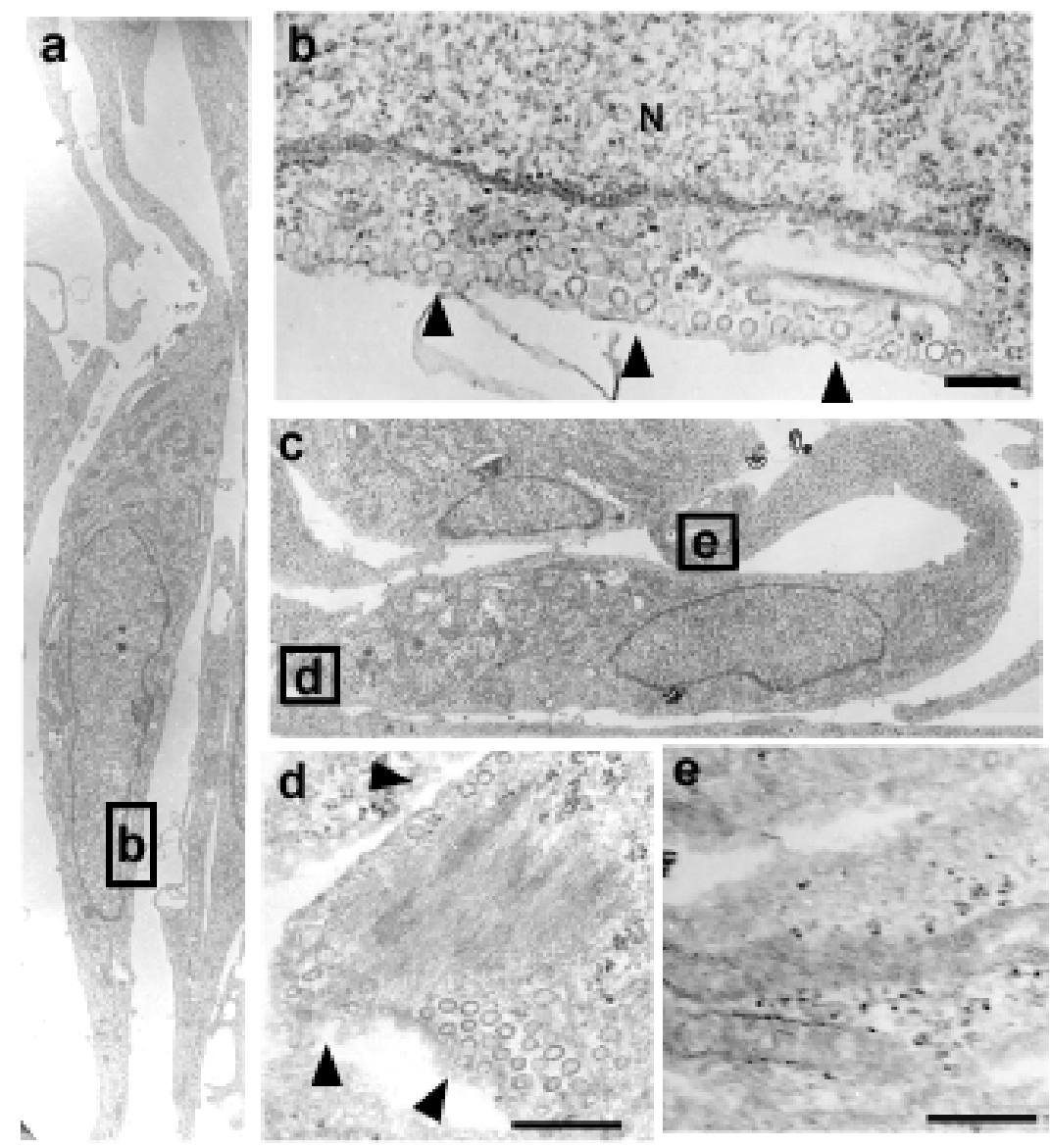

Figure 3. Exclusion of caveolae from lamellipodia. A, Endothelial cells were seeded for one hour to allow spreading $(A)$, or a confluent monolayer was scraped to induce cell migration $(C)$. After incubation, cells were fixed and processed for transmission electron microscopy. During cell spreading, caveolae, like caveolin-1, were centrally located (arrowheads in $B$ ) in proximity to the nucleus $(\mathrm{N})$ and excluded from lamellipodia. When cells were migrating, caveolae were found to be concentrated in the rear of the cells (arrowheads in $D$ ) opposite the lamellipodia $(E)$. Scale bars: $0.2 \mu \mathrm{m}$ in $B ; 0.5 \mu \mathrm{m}$ in $D$ and $E$. 
B

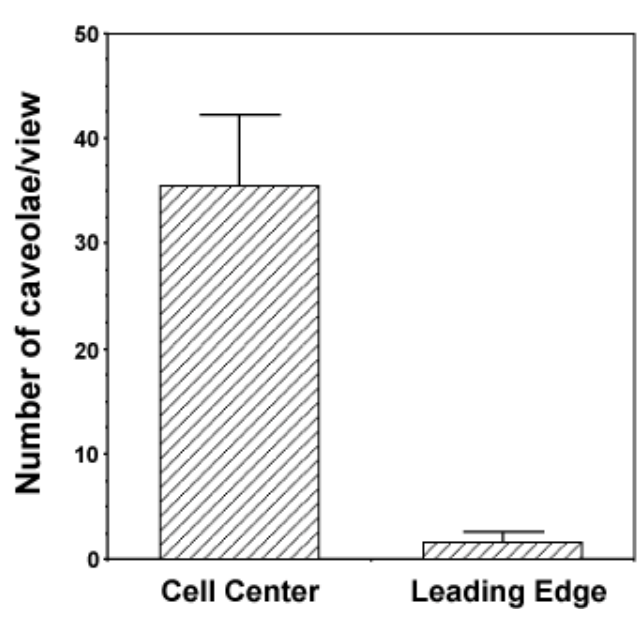

C

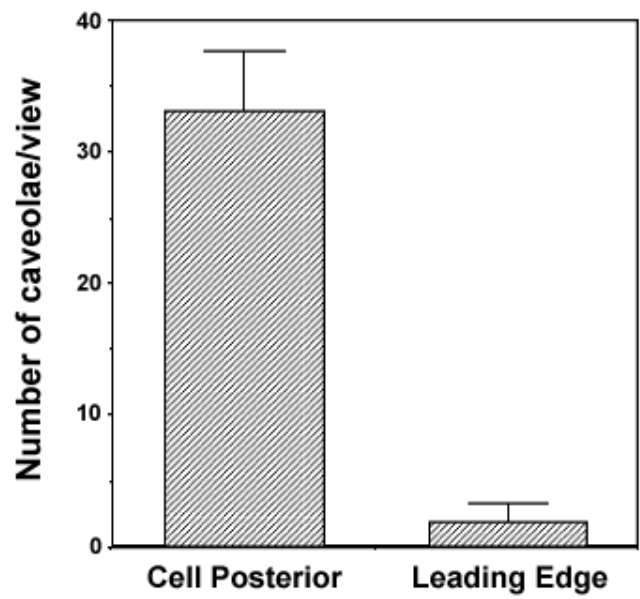

Figure 3. Exclusion of caveolae from lamellipodia. The number of caveolae at the leading edge and cell center of spreading cells (B), and the leading edge and cell posterior of migrating cells $(\mathrm{C})$ was determined by counting caveolae in randomly taken photographs. Data are the means \pm SD from ten photographs. 

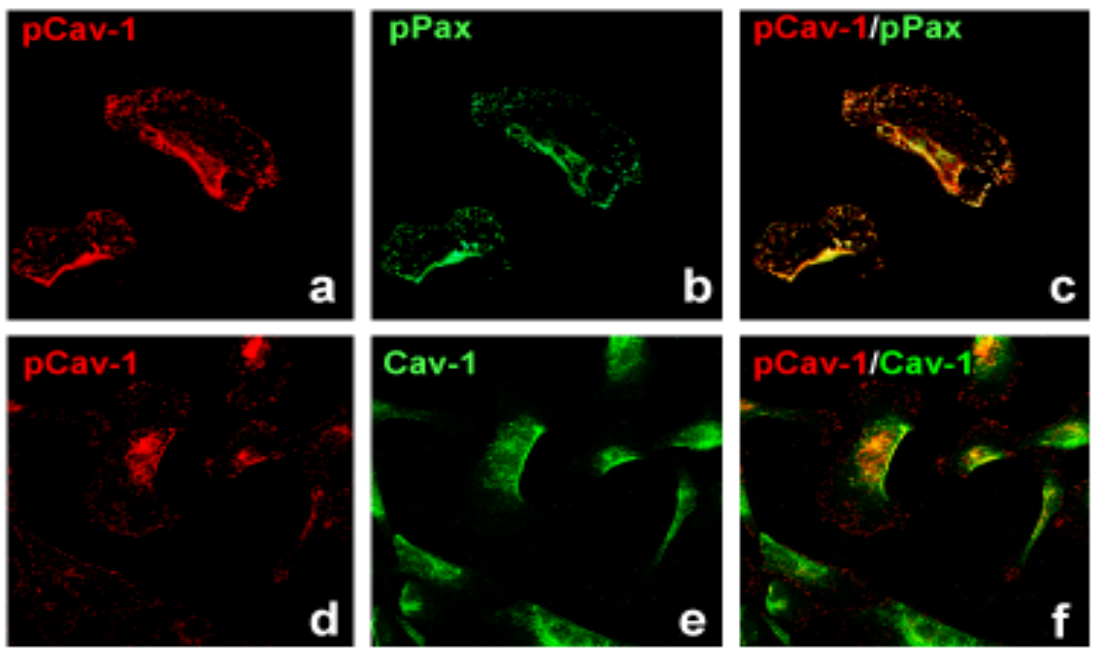

Figure 4. Co-localization of phospho-caveolin-1, but not caveolin-1, with focal complex molecules at leading edge of migrating cells. HUVECs

were seeded on fibronectin-coated coverslips and incubated to allow migration. After incubation, cells were fixed and stained with specific antibodies against phospho-caveolin-1 (pCav-1) (a,d), phospho-paxillin (pPax) (b), or Cav-1 (e). Composite images $(c, f)$ resulting from the superimposition of pCav-1 signal and pPax or Cav-1 reveal a significant co-localization of pCav-1 with pPax (c) at focal complexes. In contrast, Cav-1 was concentrated in the body and rear of migrating cells opposite pCav- 1 at the leading edge $(f)$. 

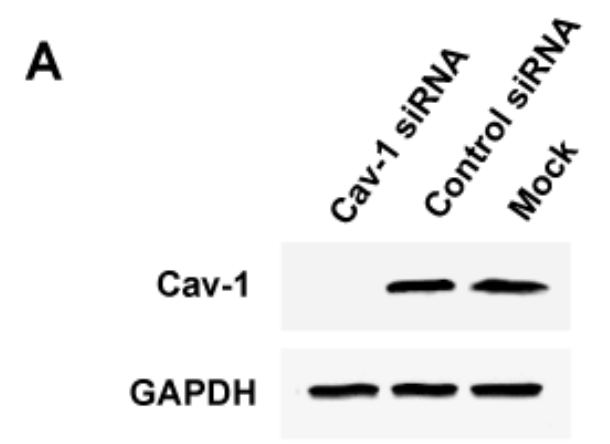

B

Mock

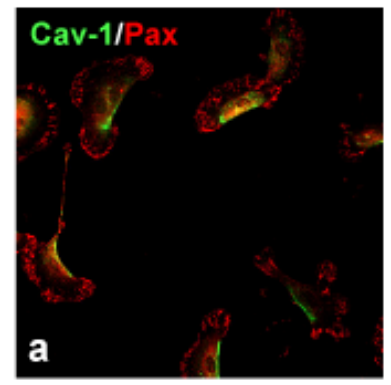

Cav-1 SiRNA

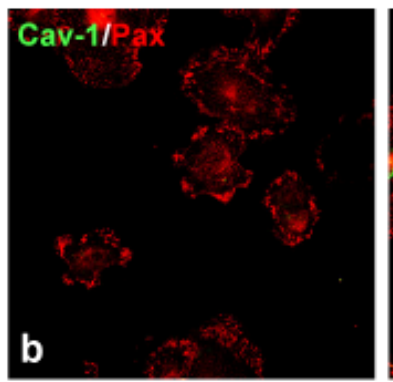

Control siRNA

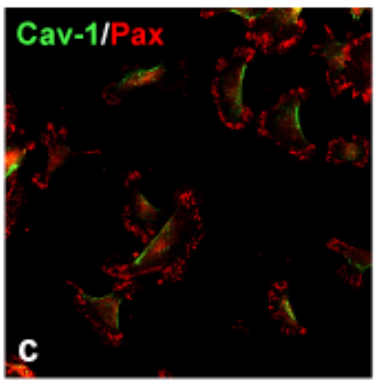

Figure 5. Knockdown of caveolin-1 prevents endothelial cell polarization. HUVECs were transfected with either caveolin-1 siRNA or control siRNA as described in Experimental Procedures. A. Caveolin-1 protein levels were specifically knocked down by the caveolin-1-specific siRNA. B. Forty-eight hours after transfection, HUVECs were seeded on fibronectin, incubated to allow migration, and then subjected to dual immunofluorescent staining with specific antibody against caveolin-1 (FITC) or paxillin (Rhodamine Red-X). Note that mock- and control siRNA-treated cells were able to polarize $(a, c)$. In contrast, knockdown of caveolin-1 impeded polarization of the cells $(b)$, which instead displayed a near-circular lamellipodium. 


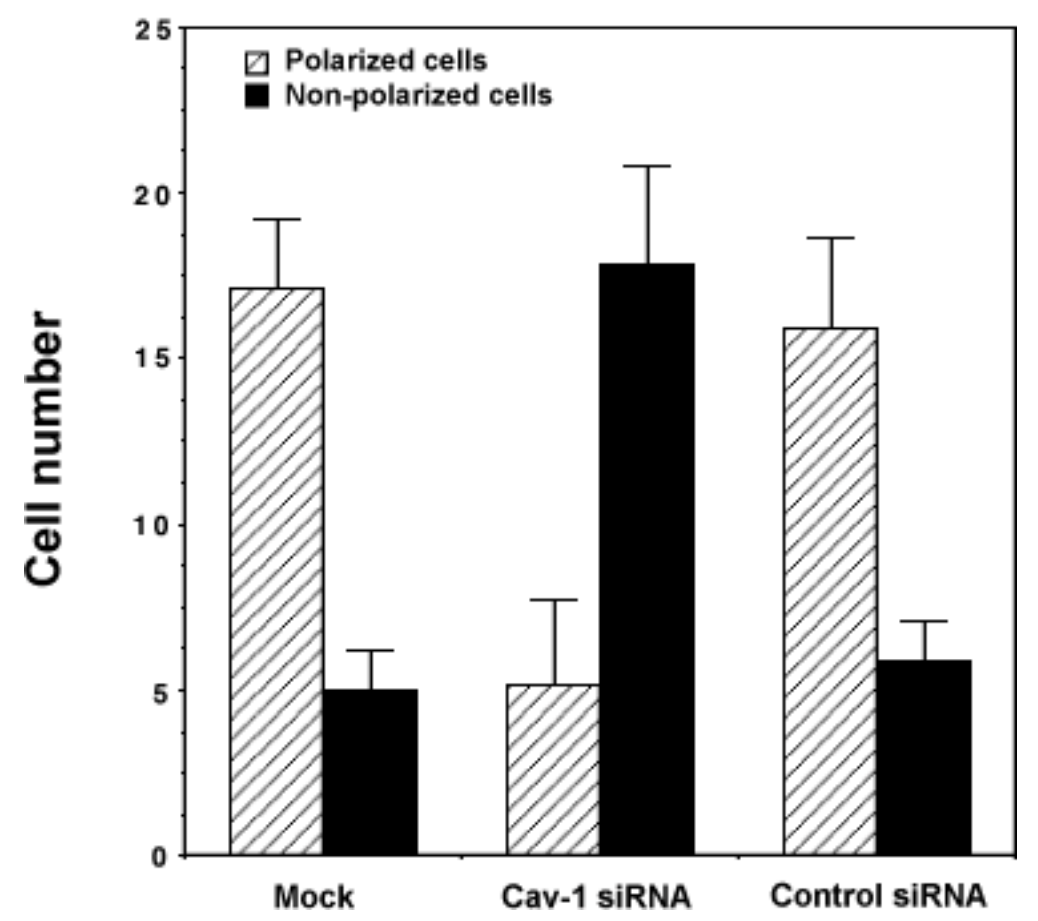

Figure 5. Knockdown of caveolin-1 prevents endothelial cell polarization. C. The effect of caveolin-1 knockdown on cell polarization was quantified by counting circular vs. polarized cells from eight randomly selected views corresponding to each of the treatments. Data are the means \pm SD. 


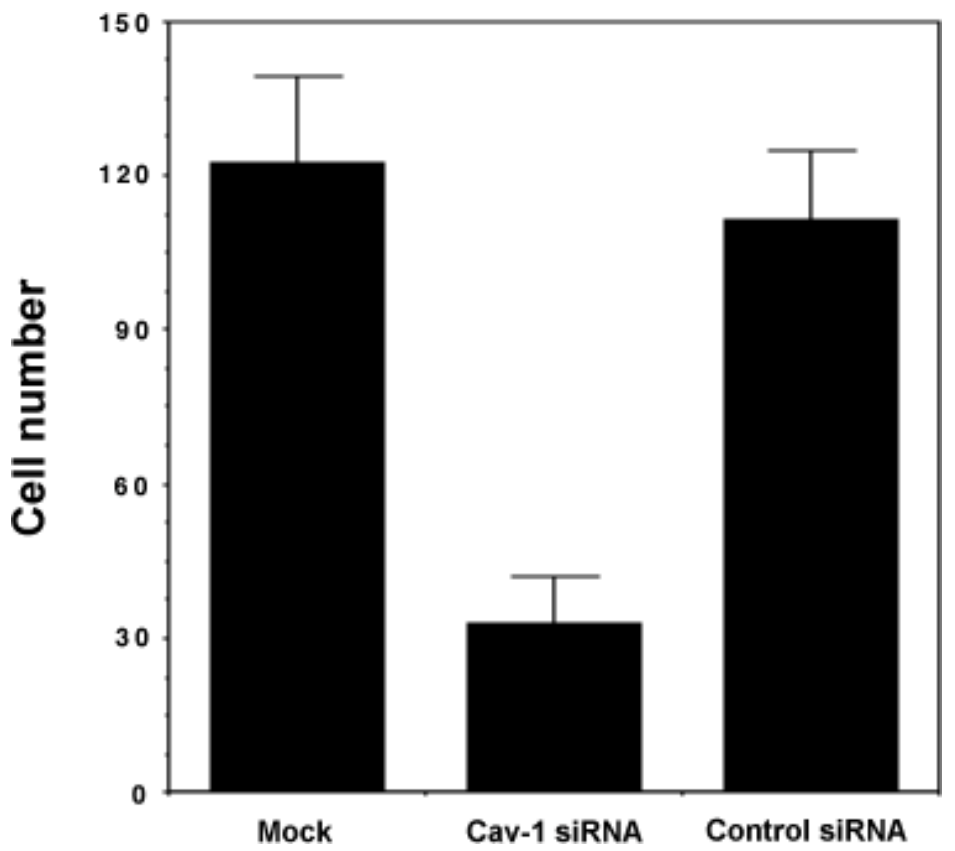

Figure 6. Knockdown of caveolin-1 inhibits endothelial cell directional movement. HUVECs were transfected with either caveolin-1-specific or control siRNA or alternately were mock transfected. Forty-eight hours after transfection, cells were placed over a polycarbonate filter and allowed to migrate through $8 \mu \mathrm{m}$ pores to an adjacent compartment in response to serum stimulation. After a 3hour incubation, cells that migrated to the serum compartment were counted. Note that knockdown of caveolin-1 dramatically inhibited cell migration compared to control siRNA- or mock-treated cells. Data are mean \pm SD. 


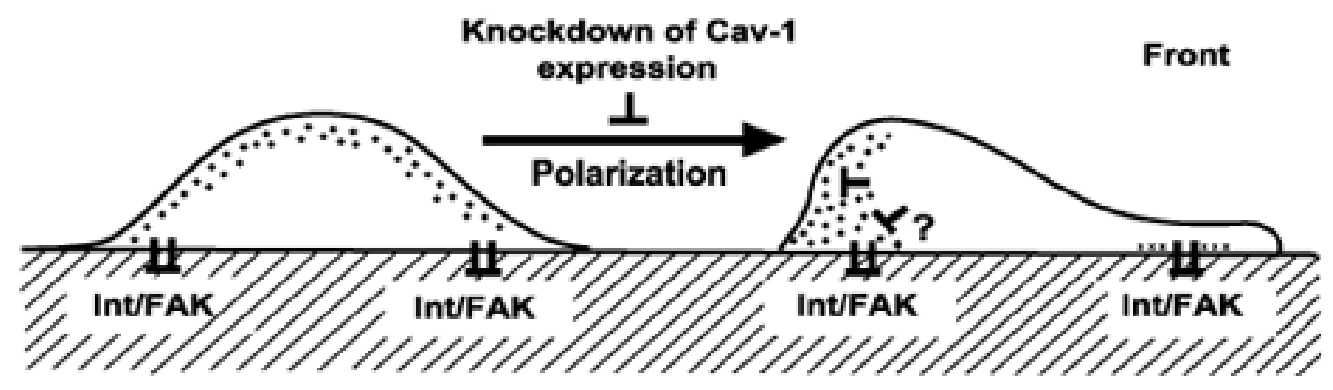

Figure 7. Proposed model demonstrating caveolin-1 polarity and control of lamellipod protrusion. Caveolin-1, the integral membrane protein of caveolae, is believed to interact with signaling molecules. Immunofluorescent staining of a resting cell (left) with antibody for caveolin-1 reveals punctate staining pattern at cell surface (small dots, left). Upon chemotactic stimulation (right), caveolin-1 is excluded from the leading edge by an unknown mechanism and relocates to the cell posterior (small dots, right) where it prevents lamellipod protrusion ( $T$ bars, right). In contrast, tyrosine phosphorylated caveolin-1 is separated from caveolae and associated with focal complexes at the leading edge (small cross, right). Thus, caveolin-1 polarity serves to spatially organize cellular activity that mediates lamellipod protrusion. Loss of caveolin-1 polarity by targeted knockdown of the protein impedes cell polarization. 


\section{REFERENCES}

Anderson, R. G. (1998). "The caveolae membrane system." Annu Rev Biochem 67: 199225.

Anderson, R. G. W. (1993). "Caveolae: Where incoming and outgoing messengers meet." Proc. Natl. Acad. Sci., USA 90: 10909-10913.

Anderson, R. G. W. (1998). "The caveolae membrane system." Ann. Rev. Biochem. 67: 199-225.

Ashton, A. W., R. Yokota, et al. (1999). "Inhibition of endothelial cell migration, intercellular communication, and vascular tube formation by thromboxane A(2)." J Biol Chem 274(50): 35562-70.

Cao, H., A. R. Sanguinetti, et al. (2004). "Oxidative stress activates both Src-kinases and their negative regulator Csk and induces phosphorylation of two targeting proteins for Csk: caveolin-1 and paxillin." Exp Cell Res 294(1): 159-71.

Conrad, P. A., E. J. Smart, et al. (1995). "Caveolin cycles between plasma membrane caveolae and the Golgi complex by microtubule-dependent and microtubuleindependent steps." J Cell Biol 131(6 Pt 1): 1421-33.

Das, K., R. Y. Lewis, et al. (1999). "The membrane spanning domains of caveolins 1 and 2 mediate the formation of caveolin hetero-oligomers. Implications for the assembly of caveolae membranes in vivo." J. Biol. Chem. 274: 18721-18726.

Folkman, J. (2002). "Role of angiogenesis in tumor growth and metastasis." Semin Oncol 29(6 Suppl 16): 15-8.

Gingras, D., F. Gauthier, et al. (1998). "Localization of RhoA GTPase to endothelial caveolae-enriched membrane domains." Biochem Biophys Res Commun 247(3): 888-93.

Glenney, J. R. and L. Zokas (1989). "Novel tyrosine kinase substrates from Rous sarcoma virus transformed cells are present in the membrane cytoskeleton." J. Cell Biol. 108: 2401-2408.

Griffoni, C., E. Spisni, et al. (2000). "Knockdown of caveolin-1 by antisense oligonucleotides impairs angiogenesis in vitro and in vivo." Biochem. Biophys. Res. Commun. 276: 756-761.

Han, D. C. and J. L. Guan (1999). "Association of focal adhesion kinase with Grb7 and its role in cell migration." J Biol Chem 274(34): 24425-30. 
Isshiki, M., J. Ando, et al. (2002). "Sites of $\mathrm{Ca}(2+)$ wave initiation move with caveolae to the trailing edge of migrating cells." J Cell Sci 115(Pt 3): 475-84.

Kasus-Jacobi, A., V. Bereziat, et al. (2000). "Evidence for an interaction between the insulin receptor and Grb7. A role for two of its binding domains, PIR and SH2." Oncogene 19(16): 2052-9.

Kawamura, S., S. Miyamoto, et al. (2003). "Initiation and transduction of stretch-induced RhoA and Rac1 activation through caveolae: cytoskeletal regulation of ERK translocation." J Biol Chem 278(33): 31111-7.

Labrecque, L., I. Royal, et al. (2003). "Regulation of vascular endothelial growth factor receptor-2 activity by caveolin-1 and plasma membrane cholesterol." Mol Biol Cell 14(1): 334-47.

Lee, H., D. Volonte, et al. (2000). "Constitutive and growth factor-regulated phosphorylation of caveolin-1 occurs at the same site (Tyr-14) in vivo: identification of a c-Src/Cav-1/Grb7 signaling cassette." Mol Endocrinol 14(11): 1750-75.

Li, S., R. Seitz, et al. (1996). "Phosphorylation of caveolin by Src tyrosine kinases: The aisoform of caveolin is selectively phosphorylated by v-Src in vivo." J. Biol. Chem. 271: 3863-3868.

Lisanti, M. P., P. Scherer, et al. (1994). "Caveolae, caveolin and caveolin-rich membrane domains: A signalling hypothesis." Trends In Cell Biology 4: 231-235.

Lisanti, M. P., P. E. Scherer, et al. (1994). "Characterization of caveolin-rich membrane domains isolated from an endothelial-rich source: Implications for human disease." J. Cell Biol. 126: 111-126.

Liu, J., P. Oh, et al. (1997). "Organized endothelial cell signal transduction in caveolae." J. Biol. Chem. 272: 7211-7222.

Liu, J., X. B. Wang, et al. (2002). "Caveolin-1 expression enhances endothelial capillary tubule formation." J Biol Chem. 277: 10661-10668.

Manes, S., E. Mira, et al. (1999). "Membrane raft microdomains mediate front-rear polarity in migrating cells." Embo J 18(22): 6211-20.

Manes, S., E. Mira, et al. (2000). "Cells on the move: a dialogue between polarization and motility." IUBMB Life 49(2): 89-96.

Meili, R. and R. A. Firtel (2003). "Two poles and a compass." Cell 114(2): 153-6.

Monier, S., R. G. Parton, et al. (1995). "VIP21-caveolin, a membrane protein constituent of the caveolar coat, oligomerizes in vivo and in vitro." Mol. Biol. Cell 6: 911927. 
Mundy, D. I., T. Machleidt, et al. (2002). "Dual control of caveolar membrane traffic by microtubules and the actin cytoskeleton." J Cell Sci 115(Pt 22): 4327-39.

Okada, S. S., J. E. Tomaszewski, et al. (1995). "Migrating vascular smooth muscle cells polarize cell surface urokinase receptors after injury in vitro." Exp Cell Res 217(1): 180-7.

Okamoto, T., A. Schlegel, et al. (1998). "Caveolins, A family of scaffolding proteins for organizing "pre-assembled signaling complexes" at the plasma membrane." J. Biol. Chem., (Mini-review) 273: 5419-5422.

Parat, M. O., B. Anand-Apte, et al. (2003). "Differential caveolin-1 polarization in endothelial cells during migration in two and three dimensions." Mol Biol Cell 14(8): 3156-68.

Peters, K.-R., W. Carley, et al. (1985). "Endothelial plasmalemmal vesicles have a characteristic striped bipolar surface structure." J. Cell Biol. 101: 2233-2238.

Pike, L. J. and L. Casey (1996). "Localization and turnover of phosphatidylinositol 4,5bisphosphate in caveolin-enriched membrane domains." J. Biol. Chem. 271: 26453-26456.

Rothberg, K. G., J. E. Heuser, et al. (1992). "Caveolin, a protein component of caveolae membrane coats." Cell 68: 673-682.

Segall, J. E., S. Tyerech, et al. (1996). "EGF stimulates lamellipod extension in metastatic mammary adenocarcinoma cells by an actin-dependent mechanism." Clin Exp Metastasis 14(1): 61-72.

Smart, E., Y.-S. Ying, et al. (1994). "Caveolin moves from caveolae to the Golgi apparatus in response to cholesterol oxidation." J. Cell Biol. 127: 1185-1197.

Song, K. S., P. E. Scherer, et al. (1996). "Expression of caveolin-3 in skeletal, cardiac, and smooth muscle cells. Caveolin-3 is a component of the sarcolemma and cofractionates with dystrophin and dystrophin-associated glycoproteins." J Biol Chem 271(25): 15160-5.

Tang, S., K. G. Morgan, et al. (1997). "Requirement for protein kinase C theta for cell cycle progression and formation of actin stress fibers and filopodia in vascular endothelial cells." J. Biol. Chem. 272: 28704-28711.

Volonte, D., F. Galbiati, et al. (2001). "Cellular stress induces the tyrosine phosphorylation of caveolin-1 (Tyr(14)) via activation of p38 mitogen-activated protein kinase and c-Src kinase. Evidence for caveolae, the actin cytoskeleton, 
and focal adhesions as mechanical sensors of osmotic stress." $\underline{\mathrm{J} \text { Biol Chem }}$ 276(11): 8094-103.

Wary, K. K., i. A. Mariott, et al. (1998). "A requirement for caveolin-1 and associated kinase Fyn in integrin signaling and anchorage-dependent cell growth." Cell 94: 625-634.

Wei, Y., X. Yang, et al. (1999). "A role for caveolin and the urokinase receptor in integrin-mediated adhesion and signaling." J Cell Biol 144(6): 1285-94.

Woodman, S. E., A. W. Ashton, et al. (2003). "Caveolin-1 knockout mice show an impaired angiogenic response to exogenous stimuli." Am J Pathol 162(6): 205968.

Zicha, D., G. A. Dunn, et al. (1991). "A new direct-viewing chemotaxis chamber." $\underline{\mathrm{J} \text { Cell }}$ Sci 99 ( Pt 4): 769-75.

Zundel, W., L. M. Swiersz, et al. (2000). "Caveolin 1-mediated regulation of receptor tyrosine kinase-associated phosphatidylinositol 3-kinase activity by ceramide." Mol Cell Biol 20(5): 1507-14. 


\section{Study 2}

Caveolin-1 Recruitment Promotes Focal Adhesion Disassembly at the Trailing Edge of Motile Cells 


\section{ABSTRACT}

The interaction of a migrating cell with extracellular substrates is mediated by a continuous and concerted assembly and disassembly of anchoring complexes known as focal adhesions at the front and rear of the cell. Although the kinetics of focal adhesion turnover are well-characterized, the molecular events that initiate adhesion disassembly remain unclear. Using live-cell imaging, we demonstrate that recruitment of caveolin-1 to focal adhesions at the cell rear is followed immediately by adhesion disassembly and trailing edge retraction. Furthermore, caveolin-1 ablation results in a decreased rate of adhesion disassembly and an increase in tail persistence in migrating fibroblasts. Our results show that caveolin-1 plays a role in the detachment of the cell trailing edge, providing a clue in the complex interactions between a cell and its substrate during cell transit. 


\section{INTRODUCTION}

Cell migration involves the complex coordination of front-rear signaling, cytoskeletal and adhesion events (Ridley, Schwartz et al. 2003; Raftopoulou and Hall 2004). It starts with the initial protrusion and extension of the plasma membrane driven by the polymerization of cortical actin filaments at the leading edge. The protrusive plasma membranes are stabilized through the formation of focal adhesions. The adhesions serve as points of traction over which the body of the cell moves. Adhesion disassembly and retraction at the cell rear are required to allow the cell to follow the extending front. The assembly of focal adhesions is initiated by the sequential recruitment of adhesion molecules around a nucleation center and is thought to be mediated by Rho GTPases (Nobes and Hall 1995; Webb, Parsons et al. 2002). In this respect, Rac functions to signal the creation of new adhesions at the leading edge, whereas Rhomediated acto-myosin contractility stimulates maturation of the adhesions (Rottner, Hall et al. 1999). In contrast, the mechanism that regulates adhesion disassembly at cell rear is poorly understood, although recent studies propose mechanisms involving proteolytic activity by calpain (Franco, Rodgers et al. 2004) and microtubule targeting to adhesions (Kaverina, Krylyshkina et al. 1999; Ezratty, Partridge et al. 2005).

Caveolae are specific microdomains at the plasma membrane involved in endocytosis, transcytosis, cholesterol homeostasis and signal transduction (Anderson 1998; Cohen, Hnasko et al. 2004). Caveolin-1 is the principal structural protein of caveolae that interacts with signaling molecules and may 
function generally as an endogenous negative regulator of signaling molecules (Williams and Lisanti 2004). Recently, we showed that caveolin-1 polarized to the cell posterior in migrating endothelial cells and that concentrations of caveolin-1 signal at the cell perimeter were devoid of focal adhesions (Beardsley, Fang et al. 2005). These results prompted us to ask whether caveolin-1 played any role in focal adhesion dynamics at the cell rear during migration. Here, we show that recruitment of caveolin-1 to trailing adhesions occurs immediately prior to adhesion disassembly. Furthermore, caveolin-1 ablation resulted in defective tail detachment in migrating fibroblasts. 


\section{EXPERIMENTAL PROCEDURES}

Reagents and expression constructs. Monoclonal antibody for paxillin was purchased from Biosource International (Camarillo, CA). Antibodies for caveolin1 were from Santa Cruz Biotechnology, Inc. (Santa Cruz, CA). The full-length cDNA encoding caveolin-1 or mutants were fused in-frame to the $\mathrm{N}$-terminus of GFP. EcoRI and BamHI restriction sites were added to 5' and 3' ends of murine caveolin-1 cDNA by polymerase chain reaction (PCR) using TripleMaster $\circledast$ PCR System (Brinkmann instruments, Inc.). The PCR products were subcloned into the EcoRI and BamHI sites of pEGFP-N1. pmRFP-N1 was generated by replacing GFP of pEGFP-N1 with mRFP (mRFP-wGBD was provided by W.M. Bement, University of Wisconsin-Madison, Madison, WI). Caveolin-1-RFP was constructed by subcloning the murine caveolin-1 cDNA into pmRFP-N1. PaxillinGFP was a generous gift from M.D. Schaller at the University of North Carolina.

Cell culture and transfection. Primary cultured endothelial cells from the human umbilical vein (HUVECs) were obtained as described previously from consenting healthy, term patients according to institutional guidelines (Beardsley, Fang et al. 2005). HUVECs were grown in MCDB supplemented with $20 \%$ heat-inactivated newborn calf serum, $5 \%$ heat-inactivated human serum and endothelial cell growth supplement.

Caveolin-1 deficient $\left(\right.$ Cav- $\left.1^{-1-}\right)$ and litter mate control wild-type MEFs were obtained from Day 13.5 embryos essentially as described (Razani, Engelman et al. 2001). Briefly, embryos were decapitated, thoroughly minced, and trypsinized 
in $1 \mathrm{ml}$ of $0.05 \%$ trypsin, $0.53 \mathrm{mM}$ EDTA (Life Technologies, Inc.) for $20 \mathrm{~min}$ at $37^{\circ} \mathrm{C}$. Fibroblasts were resuspended in complete medium (Dulbecco's modified Eagle's medium (DMEM) supplemented with 10\% fetal bovine serum (FBS), $2 \mathrm{mM}$ glutamine, $100 \mathrm{units} / \mathrm{ml}$ penicillin, and $100 \mu \mathrm{g} / \mathrm{ml}$ streptomycin (Life Technologies, Inc.) and cultured in a $37{ }^{\circ} \mathrm{C}, 5 \% \mathrm{CO}_{2}$ incubator. Primary MEFs between passages 2-5 were used for experiments.

Transient transfections of HUVECs and MEFs were performed using Lipofectin and Lipofectamine reagents (Life Technologies, Inc.), respectively, according to the protocol provided by the manufacturer. Cells were analyzed 36$48 \mathrm{~h}$ after transfection.

Microscopy and analysis. Cells expressing caveolin-1-GFP or co-expressing caveolin-RFP and paxillin-GFP were incubated on Lab-Tek II 2-well chambered coverglass. A temperature controlled heating stage, $\mathrm{CO}_{2}$ controlled incubator insert and Zeiss objective heater were used to maintain the cells at $37^{\circ} \mathrm{C}$ and $5 \%$ $\mathrm{CO}_{2}$. Images were captured using a Zeiss LSM 510 laser scanning confocal system, with an Axiovert 200M inverted microscope, $60 x$ or $40 x$ oil immersion lenses, and Argon and HeNe lasers. Fluorescent and/or DIC images were captured in 2-300 second intervals using Zeiss time-lapse software. Image analysis was performed using LSM510 software and Clmaging software (Compix Inc., Cranberry, PA). The background-corrected fluorescence intensity of GFPpaxillin incorporated into individual focal adhesions was measured over time as an index of focal adhesion disassembly. Decision Tree Analysis predicted that focal adhesions were stable at caveolin-adhesion distances greater than $300 \mathrm{~nm}$, 
whereas they rapidly disappeared at distances $<300 \mathrm{~nm}$. Color overlays were created as previously described (Smilenov, Mikhailov et al. 1999) using Clmaging software and Adobe Photoshop 6.0.

To analyze trailing fiber persistence, MEFs were seeded on chambered coverglass washed once with complete medium. Low power (20x) confocal images of live cells were captured for over $10 \mathrm{~h}$ at 2 minute intervals. Trailing fiber persistence was defined as the time interval between formation and recoil of the trailing fiber. Only those cell processes left in the wake of an advancing cell were defined as trailing edges. Cells that were nonmotile, rounded or blebbing were excluded from analysis. Trailing fibers that ripped at any point during observation were excluded from analysis.

Recoil velocity was defined as the rate at which trailing fibers recoil upon initial movement in relation to the substratum. The displacement of the recoiling fiber was recorded by measuring the distance between two points, the first representing the position of the distal end upon initial recoil, and the second representing the position where the distal tip entered the cell body at the end of recoil. This length was then divided by the time interval from initial movement to recoil into the cell body. Cell tracking was performed by recording the $X Y$ position of the cell nucleus at 30 minute intervals over a 300 minute period. All measurements were recorded using LSM510 software. All results are representative of three independent experiments. 
Immunocytochemistry. Twenty-four hours post-transfection with $\operatorname{Cav}_{61-178}$ GFP or Cav $_{1-178}$ GFP, caveolin-1 null MEFs were replated onto coverslips pre-coated with $10 \mu \mathrm{g} / \mathrm{ml}$ fibronectin. Cells were incubated to allow migration, fixed with $2 \%$ paraformaldehyde for $30 \mathrm{~min}$, and permeabilized for 10 min with $0.5 \%$ Triton X100. The cells were then blocked with $5 \%$ goat serum in PBS for $30 \mathrm{~min}$, followed by incubation with specific antibody for paxillin for 1 hour, then with TRITC-conjugated anti-mouse IgG for $30 \mathrm{~min}$, and mounted in the presence of Fluoromount-G (Southern Biotech Inc.). Immunostaining was visualized and photographed using a Zeiss LSM 510 confocal microscope. 


\section{RESULTS}

The polarization of caveolin- 1 to the rear of migrating endothelial cells is well-described (Isshiki, Ando et al. 2002; Beardsley, Fang et al. 2005). Caveolin1 appears in association with actin bundles as a stripe along the cell rear, opposite from areas of lamellipodial protrusion. Notably, this area is largely devoid of focal adhesion staining, curving inward presumably as a result of locally unopposed acto-myosin contraction. Though the spatial relationship of polarized caveolin-1 with the actin cytoskeleton and focal adhesions has been documented, little is known about the events leading up to these phenomena. We thus used live cell imaging to explore caveolin-1 polarization in the context of cell motility.

Human umbilical vein endothelial cells (HUVECs) expressing caveolin-1GFP were visualized by DIC and fluorescence confocal microscopy as they were crawling on fibronectin-coated coverglass. We found that caveolin-1-GFP polarized to the rear of HUVECs, colocalizing with endogenous caveolin-1 (Figure 1). As migrating cells advance, cell-matrix attachments at the cell posterior lag behind, forming a trailing edge in the wake of the cell (Chen 1981). Many such trailing fibers transiently formed and recoiled into the cell body during our observation of motile HUVECs. Composite Z-stacked time series images of a HUVEC transition from spreading to migration demonstrate polarization of the molecule concomitant with cell polarization (Figure 2a,b). As one pole of the cell initiates a forward movement, many small, trailing fibers simultaneously form at the opposite pole (Box $b$ in $\mathrm{t}=18$, Figure 2a). Intriguingly, caveolin-1-GFP signal 
is detected in these small fibers immediately prior to and during their release into the cell body (arrows, Figure 2b). Subsequently, the caveolin-1 signal appears typically polarized at the nascent cell rear (arrows in $\mathrm{t}=27$, Figure $2 b$ ). Later in the same time series, the cell shifts direction, and this movement creates trailing fibers in a new area of the cell perimeter (Box $c$ in $t=31.5$, Figure 2a). Again, caveolin-1-GFP is detected in the trailing fibers prior to and during their recoil into the cell body. Thus, formation of the small trailing fibers preceded caveolin-1 recruitment in both instances. Surprisingly, we observed that the caveolin-1-GFP signal in the trailing fibers briefly but distinctly appears in a focal adhesion pattern upon cell retraction (arrows, Figure 2c).

Next we analyzed HUVECs expressing caveolin-1-GFP that had already polarized as the cells were crawling on fibronectin-coated coverglass. Again, many trailing fibers formed and recoiled into the cell body during our observation. Strikingly, recruitment of polarized caveolin-1-GFP into these trailing fibers immediately preceded their retraction into the cell body (Figure 3). Sequential images from the time series reveal that a few small trailing fibers are formed as the cell turns approximately $45^{\circ}$ to its right (panel a, Figure 3 ). Caveolin-1-GFP concentrates in the fibers just prior to their release from the fibronectin matrix and retraction into the cell. Another example is shown in Figure 3, panel $b$. Here caveolin-1 advances across the perimeter of a large trailing fiber, concomitant with sequential release of the fiber into the cell body. Thus, sequential caveolin-1 advancement occurs concomitantly with step-wise release of the trailing fibers into the cell body during migration. 
The disassembly of focal adhesions at the rear of migrating cells is well described (Horwitz and Parsons 1999; Webb, Parsons et al. 2002), and presumably acts to "unpin" the trailing edge, causing it to recoil into the cell body. We hypothesized that caveolin-1 recruitment elicits recoil of the trailing edge by weakening or dispersing focal adhesions that persist at the distal end of such trailing strands. To test whether caveolin-1 recruitment precedes adhesion disassembly, we observed HUVECs co-expressing paxillin-GFP as a specific focal adhesion marker (Huang, Rajfur et al. 2003; Webb, Donais et al. 2004) and red fluorescent protein (RFP) tagged-caveolin-1 as the cells were crawling on fibronectin. The polarization of caveolin-1-RFP and caveolin-1-GFP was comparable, demonstrating that RFP-tagged caveolin-1 was suitable for use in this experiment (unpublished data).

Live-cell imaging revealed that recruitment of caveolin-1 to focal adhesions at the cell rear occurred immediately prior to their disassembly, indeed suggesting that caveolin-1 recruitment may be an initiating event in adhesion disassembly. Sequential images from the time series are shown in Figure $4 a$. Caveolin-1 was false-colored green and paxillin red for consistency. The basolateral edge of a migrating HUVEC is shown retracting as the cell advances. Polarized caveolin-1 was initially located at the cell rear adjacent to focal adhesions that lined the basolateral edge (arrows, lower panel in Figure 4a). Disassembly of the focal adhesions, evidenced by the dispersion of the paxillinGFP signal, occurred following the recruitment of caveolin-1 into the adhesions (arrowhead and asterisk, lower panel in Figure 4a). Those focal adhesions not in 
immediate proximity with caveolin-1 were not affected in the time frame shown (circle, lower panel in Figure 4a), but eventually disassembled upon caveolin-1 recruitment. DIC images reveal that recoil of the trailing edge occurs simultaneously with the loss of paxillin (upper panel in Figure 4a), thus indicating complete disassembly of the focal adhesions rather than loss of paxillin per se from the adhesions.

To graphically represent the relationship between caveolin-1 recruitment and focal adhesion disassembly, the fluorescent intensities of paxillin-GFP positive trailing edge adhesions were plotted as a function of distance from caveolin-1 (Figure 4c). At caveolin-adhesion distances beyond $300 \mathrm{~nm}$, or approximately 3-6 caveolae diameters, the adhesions were relatively stable. As caveolin-1-RFP came within proximity $(\leq 300 \mathrm{~nm})$ of an adhesion, however, paxillin fluorescent intensities began to sharply decrease, confirming that adhesion disassembly occurred only upon recruitment of caveolin-1 to the immediate vicinity of the adhesion.

The development of intracellular tension by the actin cytoskeleton creates a traction force on focal complexes and induces their maturation into higher density focal adhesions (Chrzanowska-Wodnicka and Burridge 1996; Galbraith and Sheetz 1998). We reasoned that a reduction of traction force may conversely initiate adhesion disassembly. Intriguingly, Smilenov et al. (Smilenov, Mikhailov et al. 1999) have demonstrated a sliding of focal adhesions relative to the substratum during cell retraction, suggesting a reduction in traction force on such adhesions could occur via inside-out weakening of the adhesion-matrix 
interaction. This prompted us to determine whether caveolin-1 recruitment coincided with adhesion sliding through weakening of focal adhesion avidity for fibronectin. To visualize sliding, we created an overlay from the previous time series using different colors to represent sequential frames (Figure 4b). An overlay of frames taken before, during, and immediately after caveolin-1 recruitment to FA1 reveal that sliding of the adhesion began with caveolin recruitment and occurred simultaneously with disassembly. In contrast, sliding of adhesions FA2-4 was negligible in the time frame shown, but occurred upon caveolin-1 recruitment (data not shown). Thus, focal adhesion sliding occurred, suggesting that weakening of adhesion-matrix interaction coincident with caveolin-1 recruitment may contribute to adhesion disassembly.

The formation of trailing fibers prior to caveolin-1 recruitment presumably results from the initial resistance of distal adhesions to acto-myosin contraction (Figure 5a). In attempt to observe recoil of the trailing edge while simultaneously visualizing caveolin and the core actin cytoskeleton, live cell imaging of motile HUVECs co-expressing $\beta$-actin-RFP (shown in blue) and caveolin-1-GFP was performed. Sequential time series images of a recoil event are shown in Figure $5 b$. Actin stress fibers arising from the cell body course through the trailing edge and terminate at the cell periphery. Although not initially present, caveolin-1-GFP begins to appear at the cell perimeter strained by the actin stress fibers (see Figure 5b). Significantly, caveolin-1 recruitment coincides with recoil of the trailing edge toward the cell body, eventually colocalizing with aggregated actin bundles in a polarized stripe at the cell rear. Interestingly, the caveolin-1 signal 
originally appears at the tips of actin stress fibers that are oriented in parallel to the direction of recoil of the trailing edge. Visualization of the actin-RFP upon tail recoil reveals coalescence of the actin bundles, reminiscent of the collapse of an accordion gate. The caveolin-1 and actin signals subsequently appear colocalized in the well-described association at the cell rear (Rothberg, Heuser et al. 1992; Stahlhut and van Deurs 2000; Isshiki, Ando et al. 2002). Thus, an interesting implication is that localized acto-myosin contraction introduces a strain on trailing edge adhesions that might serve as a specific stimulus for caveolin-1 recruitment and/or function at the cell perimeter. Consistent with this, we have found that inhibition of nonmuscle myosin II by blebbistatin significantly reduces polarization of caveolin-1 in HUVECs (see Study Three).

To determine if caveolin-1 was critically important in tail detachment, we next compared focal adhesion dynamics and persistence of the trailing edge in caveolin-1 null and wild-type mouse embryonic fibroblasts (MEFs). Importantly, caveolin-1-GFP was recruited into the trailing edge of wild-type MEFs prior to tail recoil, indicating that this function of caveolin-1 was conserved in at least two different cell types (data not shown).

We predicted that trailing edges formed during cell advancement would persist longer in $\mathrm{Cav}^{-/-} \mathrm{MEFs}$ relative to wild-type cells. We monitored trailing edge dynamics of control $(n=30)$ and mutant $(n=49)$ MEFs over 10 hours by livecell imaging. Persistence of the trailing edge was defined as the time interval between formation and recoil of the trailing edge, excluding instances of ripping of the cell from its distal attachments (see Methods). $\mathrm{Cav}^{-/-}$MEFs demonstrated 
a significant increase in persistence of the trailing edge compared to control cells, with average durations of $92 \pm 8$ and $58 \pm 8$ minutes, respectively (Figure 6a). A deficit in contractility in the mutant cells may independently cause an increase in persistence of the trailing edge. To assess contractility, we measured recoil velocity, defined as the rate at which trailing fibers recoil upon initial detachment and movement in relation to the substratum. The average recoil velocities of mutant and control cells were not significantly different (Figure 6b), suggesting that a deficit in contractility is not the cause of increased persistence of the trailing edge in mutant cells.

Examples of adhesion disassembly and tail recoil in $\mathrm{Cav}^{-/ 2}$ and control MEFs are shown in Figure 6c. A control MEF expressing paxillin-GFP undergoes adhesion disassembly and nearly complete tail recoil within one hour. In contrast, trailing edge adhesions in a caveolin-1 null MEF (arrowheads) predominantly remained stable in an equivalent time period. Moreover, color overlays of the images demonstrate significant contact plasticity in the wild-type cell whereas only a few adhesions in the caveolin-1 null cell exhibit a modest sliding (arrowheads).

To investigate the effect of caveolin-1 ablation on cell motility, we monitored the migration paths of MEFs over a 5 hour period at 30 minute intervals. Mutant cells demonstrated a significant reduction in scalar motility (total distance/interval) compared to control cells, with average speeds of $0.36 \pm$ $0.03 \mu \mathrm{m} \mathrm{min}{ }^{-1}$ and $0.47 \pm 0.04 \mu \mathrm{m} \mathrm{min}^{-1}$, respectively (Figure $7 \mathrm{a}$ ). Vectorial movement (net distance/interval) was not significantly different, however, with 
mutant and control cells having average velocities of $0.25 \pm 0.02 \mu \mathrm{m} \mathrm{min}^{-1}$ and $0.26 \pm 0.03 \mu \mathrm{m} \mathrm{min}{ }^{-1}$, respectively (Figure $7 b$ ). From 19 mutant and 27 control cells analyzed, six representative paths from each group are shown, in which control cell paths tended to be more tortuous compared to mutant cells (Figure $8 a, b)$. Interestingly, a recent report by Rid et al. suggested that anchorage of the cell rear was necessary for continuous unidirectional movement of fibroblasts (Rid, Schiefermeier et al. 2005). Thus, persistence of the trailing edge in the face of caveolin-1 ablation may decrease movement plasticity and overall chemokinesis in mutant cells. 


\section{DISCUSSION}

Detachment during cell motility is a complex process, coordinated with advancement of the leading edge and likely involving many different mediators (Webb, Parsons et al. 2002; Ridley, Schwartz et al. 2003). Taken together, the results presented here suggest that caveolin-1 participates in recoil of the trailing edge during cell migration through focal adhesion weakening and disassembly. How caveolin-1 might mediate this chaotropic effect on adhesion architecture is not known; however, adhesion disassembly occurs at caveolin-adhesion distances less than $300 \mathrm{~nm}$, suggesting that caveolin may function directly at or in the vicinity of the focal adhesion, rather than via a diffusible intermediate.

Upon recruitment to an adhesion, caveolin-1 initiates disassembly by an unknown mechanism, possibly involving an inside-out weakening of focal adhesion avidity as evidenced by adhesion sliding that occurs simultaneously with breakdown. Although caveolae have been historically defined as flaskshaped invaginations (Stan 2005), they are believed to assume a variety of shapes, including a flattened morphology at the plasma membrane (Shaul and Anderson 1998). Initial arrangement of a flattened caveolus at a focal adhesion, followed by transition to a flask-shaped morphology may disrupt focal adhesion integrity through displacement of integrin heterodimers from their ligands. Thus, although our data were collected through observation of the fluorescent-tagged molecule, the primacy of caveolin-1 or caveolae in regards to adhesion breakdown remains to be determined. 
Our results suggest acto-myosin tension exerts traction on trailing adhesions prior to caveolin-1 recruitment, indicating that such traction, or "adhesion priming", is prerequisite or contextually important in the caveolinmediated adhesion disassembly. Caveolin recruitment upon cell contraction seems to short-circuit the well-described adhesion maturation that occurs upon increase in cytoskeletal tension (Ballestrem, Hinz et al. 2001), resulting instead in adhesion disassembly, inward collapse of the strained trailing edge, and nascent association of caveolin with actin stress fibers at the cell rear. The stable association of caveolae/caveolin with the actin cytoskeleton is well-documented (Rohlich and Allison 1976; Peters, Carley et al. 1985; Rothberg, Heuser et al. 1992; Fujimoto, Miyawaki et al. 1995; Stahlhut and van Deurs 2000; Isshiki, Ando et al. 2002); moreover, caveolin-1 is thought to be physically linked and immobilized on actin stress fibers through interaction with filamin (Stahlhut and van Deurs 2000; Thomsen, Roepstorff et al. 2002; Hommelgaard, Roepstorff et al. 2005). Using live cell imaging, we have demonstrated the proximal events leading to actin-caveolin co-alignment at the cell rear upon recoil of the trailing edge. We have shown that during recoil of the trailing edge, 1) actin stress fibers stably associate with and strain focal adhesions; 2) caveolin-1 briefly and catastrophically associates with focal adhesions at the distal tip of the actin fibers; and 3) caveolin-1 stably associates with actin stress fibers at the newly-formed cell rear. Chen et al. have recently reported that the adhesion protein vinculin loses its actin-bound conformation upon focal adhesion disassembly (Chen, 
Cohen et al. 2005). It is thus interesting to speculate that caveolin may compete with adhesion proteins in binding actin, potentially provoking disassembly.

We have shown a significant defect in adhesion disassembly in $\mathrm{Cav}^{-/-} \mathrm{MEFs}$. Given the observation that recoil of the trailing edge still occurs in caveolin-1 null cells, several complementary mechanisms likely act to initiate focal adhesion disassembly and tail recoil, namely calpain-dependent proteolysis of adhesion molecules (Carragher, Fincham et al. 2001; Franco, Rodgers et al. 2004) and microtubule targeting (Kaverina, Krylyshkina et al. 1999). Moreover, ripping of the cell from points of attachment is well described (Mayer, Maaser et al. 2004; Webb, Donais et al. 2004), and was observed frequently in caveolin-1 deficient and control MEFs in the current study (data not shown). Caveolin-1 recruitment may be among the most expedient of the mechanisms, presumably involving neither the destruction of adhesion molecules nor the cell itself, and thus may be of practical importance in view of the perpetual trailing edge detachment necessary for cell migration.

Much effort has been directed towards the elucidation of focal adhesion dynamics during cell locomotion. Herein, we have described a unique example of a molecule translocation eliciting a cell movement. The contribution of caveolin-1 to focal adhesion disassembly at the rear of moving cells is thus an important clue in the complex interaction of a motile cell with its substrate. 

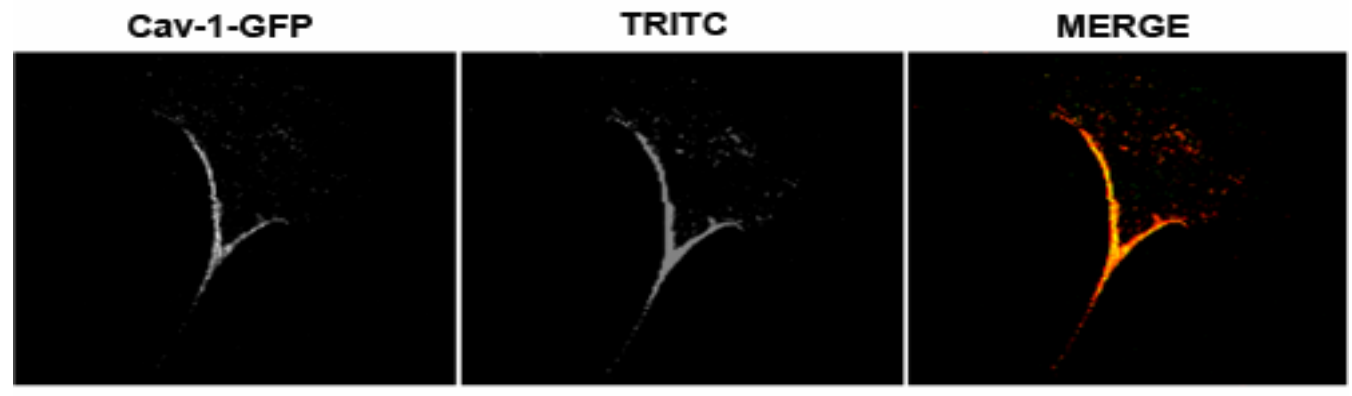

Figure 1 Caveolin-GFP colocalizes with endogenous caveolin-1 at the cell rear. Caveolin-GFP was expressed in HUVECs which were subsequently seeded and allowed to migrate on fibronectin. The cells were then fixed and stained with caveolin-1 antibody (red) which recognizes both endogenous and GFP-tagged caveolin-1. The merged image demonstrates colocalization of the signals at the cell rear, although caveolin-1 antibody signal was detected more distally in the trailing edge than the caveolin-GFP signal. 


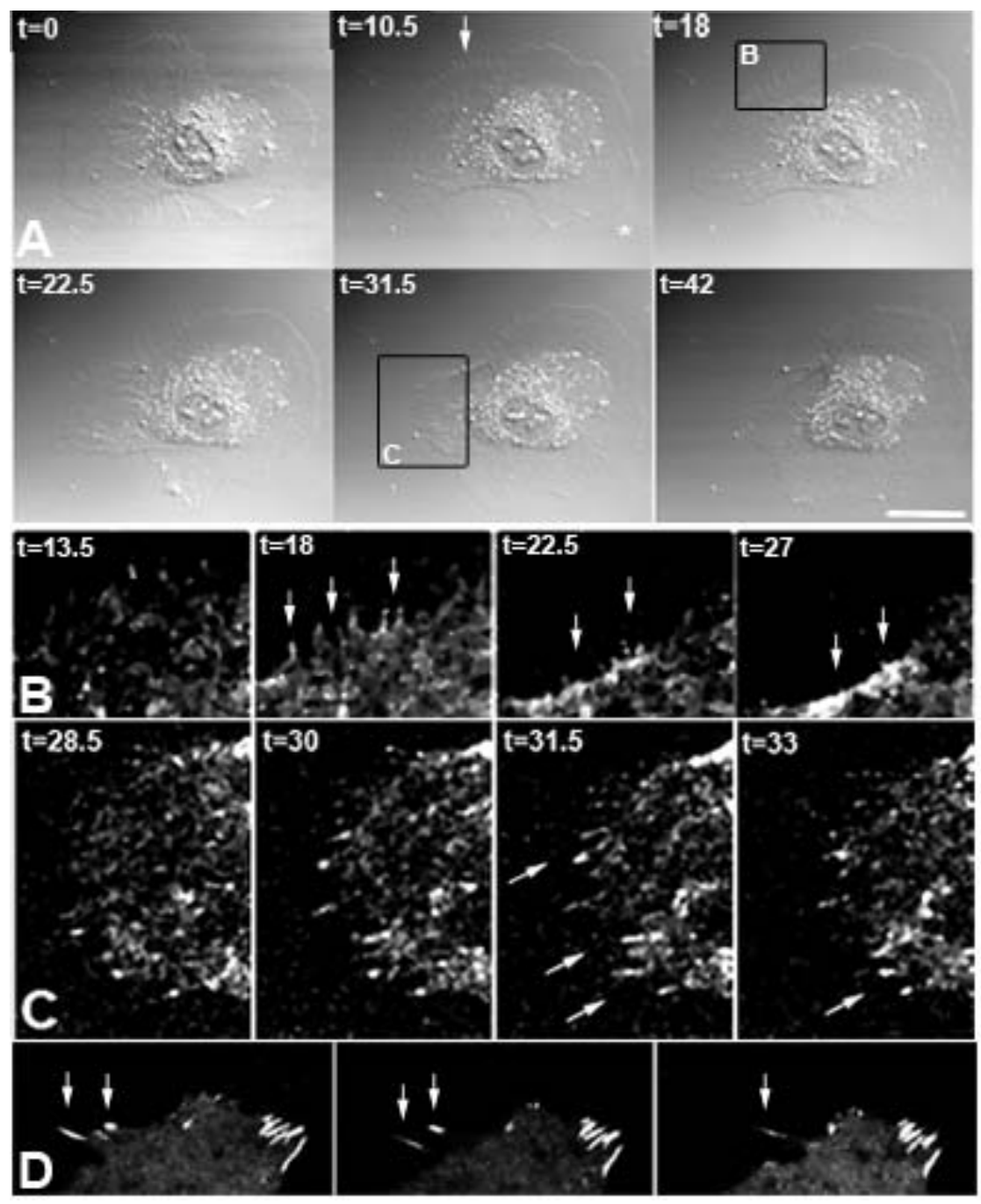

Figure 2 Polarization of caveolin-1 occurs during cell polarization. HUVECs transfected with caveolin-1-GFP were allowed to spread briefly on fibronectincoated cover glass ( $\sim 5$ minutes after seeding) were subsequently visualized by time-lapse fluorescence and differential interference contrast (DIC) microscopy. (a) Sequential DIC images demonstrate lamellipodial expansion downward and to the right of the field (asterisk, $t=10.5$ ) coupled with recoil of the cell rear in the same direction. Cell recoil results in the formation of many small trailing fibers

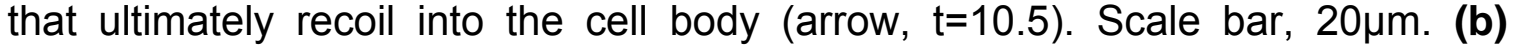
Caveolin-GFP fluorescence is shown in an enlargement of the boxed area in (A). Caveolin-GFP is detected in the nascent trailing fibers concomitant with their release into the cell body (arrows). As the trailing fibers recede, caveolin-GFP fluorescence appears polarized at the nascent cell rear (arrows, $t=27$ ). (c) Enlargement of a second recoil event reveals concentration of caveolin-1-GFP in the trailing edge, specifically in a transient focal adhesion pattern that dissipates with release into the cell body. (d) Focal adhesion disassembly in a HUVEC transiently expressing paxillin-GFP is shown for comparison (arrows). 

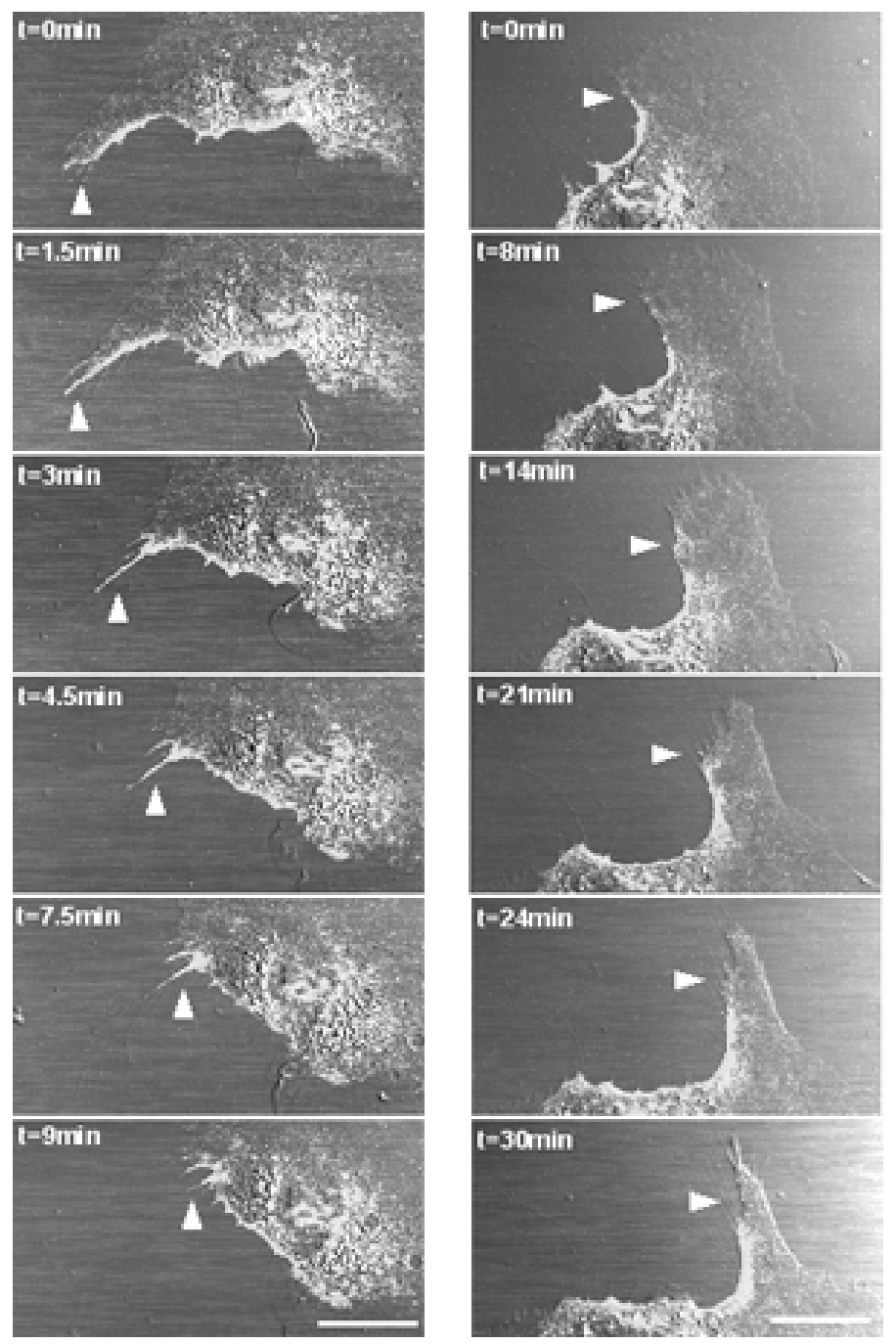

Figure 3 Caveolin-1 recruitment precedes the recoil of trailing fibers during cell migration. HUVECs transfected with caveolin-1-GFP were seeded on fibronectin-coated chambered cover glass, and images from two separate recoil events $(A \& B)$ that occurred during cell migration were taken by time-lapse fluorescence microscopy and differential interference contrast (DIC). In the first series (panel A), caveolin-1-GFP was concentrated into trailing fibers (arrowheads) that formed as the cell turned to its right. Caveolin-1 recruitment occurs immediately prior to recoil of the trailing fiber into the cell body. In the second series (panel B), a large trailing fiber persisting in the wake of the advancing cell was sequentially released as caveolin-1-GFP moved across the perimeter of the fiber (arrowheads). Scale bars $20 \mu \mathrm{m}$. 

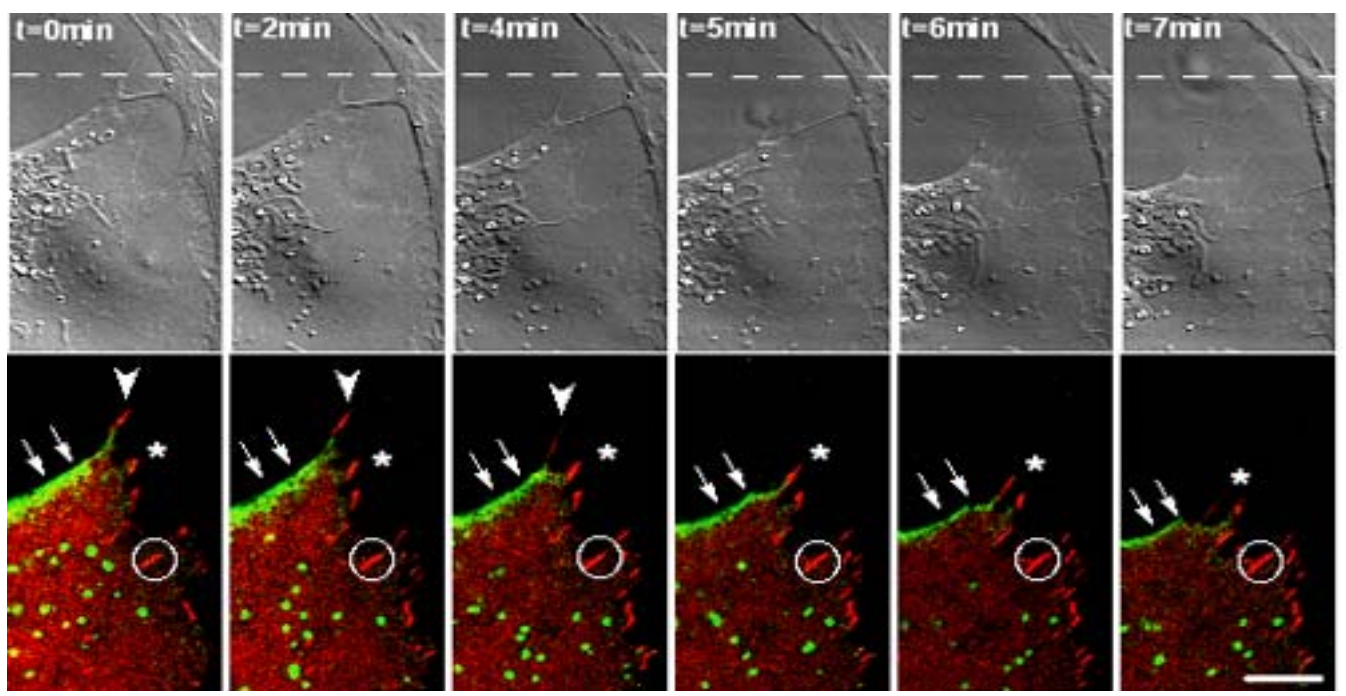

Figure 4 Caveolin-1 recruitment leads to focal adhesion disassembly and sliding in the trailing edge. (a) Live cell imaging was performed on HUVECs expressing caveolin-1-RFP and GFP-paxillin during migration on fibronectin. Caveolin-1 was false-colored green and paxillin red for consistency. Sequential frames taken from a time series demonstrate retraction of the trailing edge of a migrating cell. Several adhesions were initially $(\mathrm{t}=0 \mathrm{~min})$ positioned along the basolateral edge of the cell (arrowhead, asterisk, circle, lower panel), juxtaposed with caveolin-1 (arrows, lower panel) concentrated at the cell rear. Disassembly of two adhesions (arrowhead and asterisk), evidenced by loss of paxillin fluorescence, occurred sequentially in the order that caveolin-1 (arrows) was recruited. Those adhesions not in immediate proximity with caveolin-1 did not disassemble in the time frame shown (circle). DIC imaging (upper panel) demonstrates the retraction of the trailing edge concomitant with loss of paxillin signal, verifying adhesion disassembly rather than photobleaching or loss of paxillin per se from the adhesion (dashed line indicates a fiduciary mark). Scale bar, $10 \mu \mathrm{m}$. 


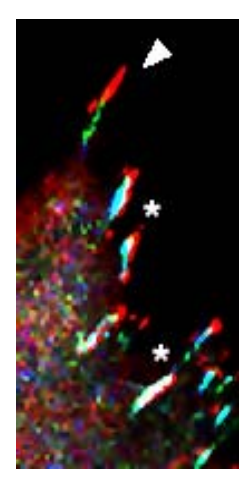

Figure 4 Caveolin-1 recruitment leads to focal adhesion disassembly and sliding in the trailing edge. (b)Three-color overlay of sequential frames (red, green, and purple)from the previous time series reveals sliding of an adhesion associated with caveolin-1 (arrowhead). The sliding adhesion appears as a rainbow in the overlay, whereas adhesions not associated with caveolin-1 (asterisks) appear as a single color (white or turquoise). Adhesion sliding occurs simultaneously with adhesion breakdown (see text). 


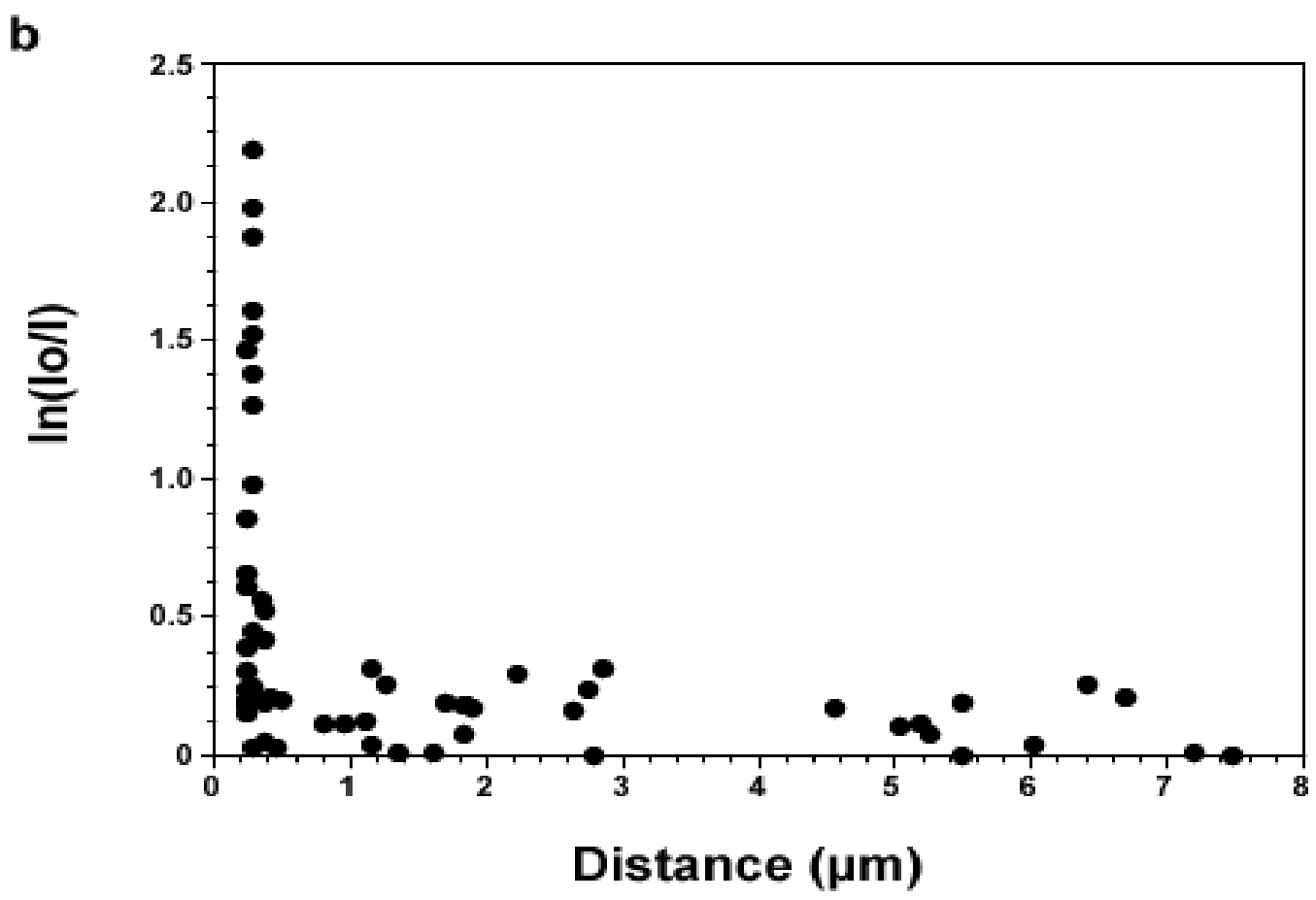

Figure 4 Analysis of trailing adhesion disassembly as a function of caveolin-1 distance. $I_{0}$ is the initial measured fluorescent intensity and $I$ the fluorescent intensity of focal adhesions at given distances from caveolin-1-RFP. The logarithm of the ratio $I_{0} / l$ is plotted as a function of adhesion distance from caveolin. Adhesions remained stable at caveolin-1 distances greater than $300 \mathrm{~nm}$, or roughly 3-6 caveolae diameters. At caveolin-adhesion distances of less than 300nm, GFP-paxillin fluorescent intensities began to decrease precipitously. Results represent analysis of ten trailing edge adhesions from four different cells. 

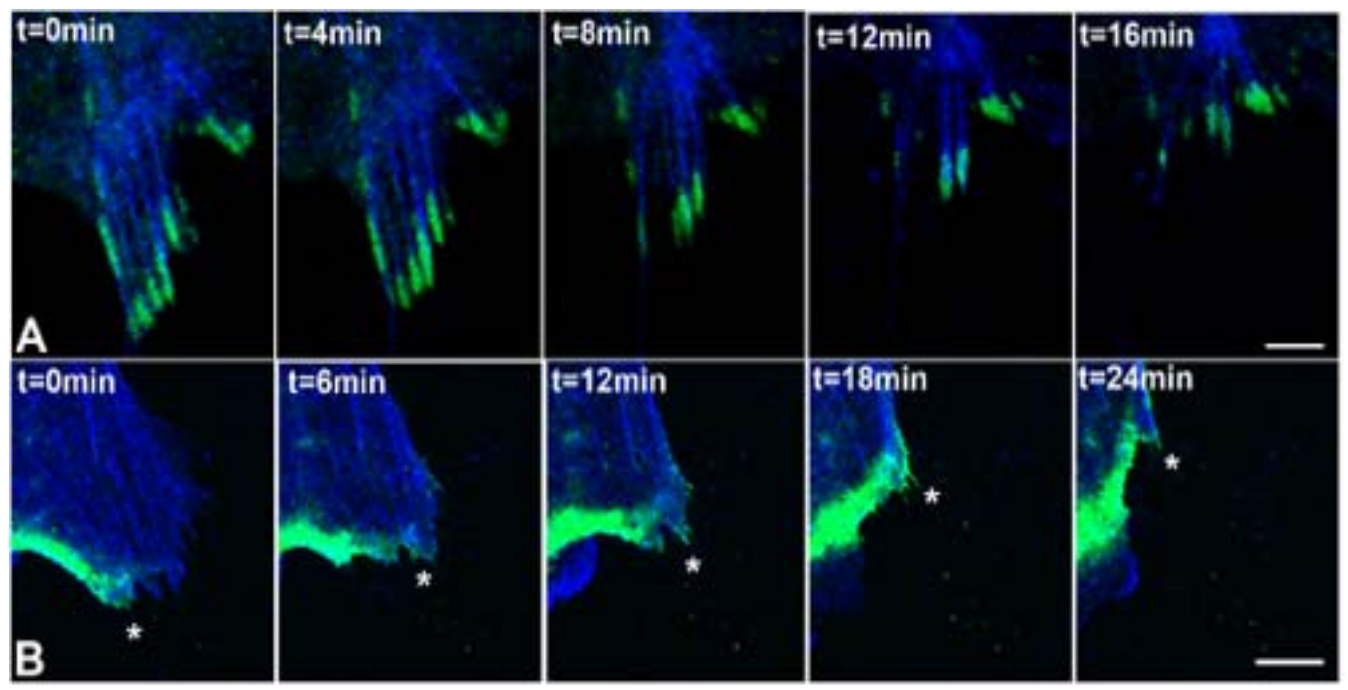

Figure 5 Involvement of the actin cytoskeleton in recoil of the trailing edge. (a) Simultaneous expression of RFP- $\beta$-actin (blue) and GFP-paxillin (green) demonstrate parallel actin bundles terminating distally at trailing edge adhesions. Sequential time series images demonstrate adhesion disassembly accompanies recoil of the actin bundles into the cell. (b) Live cell imaging was performed on HUVECs expressing caveolin-1-GFP (green) and RFP- $\beta$-actin (blue) during migration on fibronectin. Sequential images taken from a time series demonstrate retraction of the trailing edge of a migrating cell. Actin stress fibers can be seen coursing through the cell and terminating at the cell perimeter. Initially devoid of caveolin signal, the cell rear recedes as caveolin-1-GFP advances across its perimeter (asterisks). Note the spatial and temporal recruitment of caveolin-1 signal prior to withdrawal of the trailing edge. 


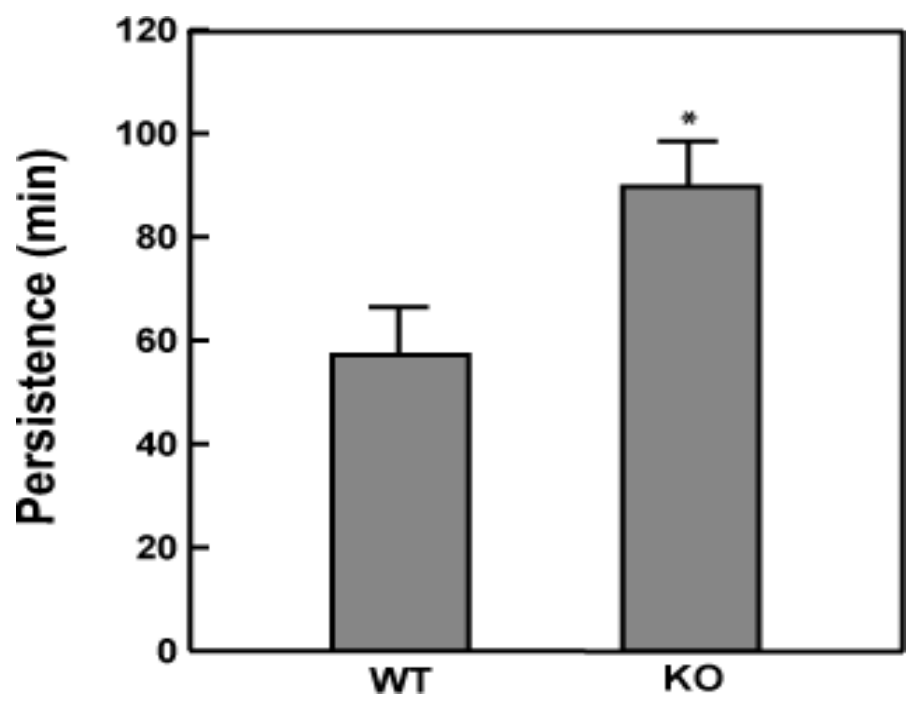

Figure 6 Impaired focal adhesion disassembly at cell trailing edges in Cav 1- MEFs. (a) Increase in trailing fiber persistence in caveolin-1 deficient MEFs. Caveolin-1 knockout or wild-type mouse embryonic fibroblasts were seeded on chambered cover glass and visualized by time-lapse DIC microscopy. Persistence of the trailing edge was determined as described in Methods. Data are the mean \pm s.e.m. from three independent experiments. ${ }^{*}, P<0.01$. 


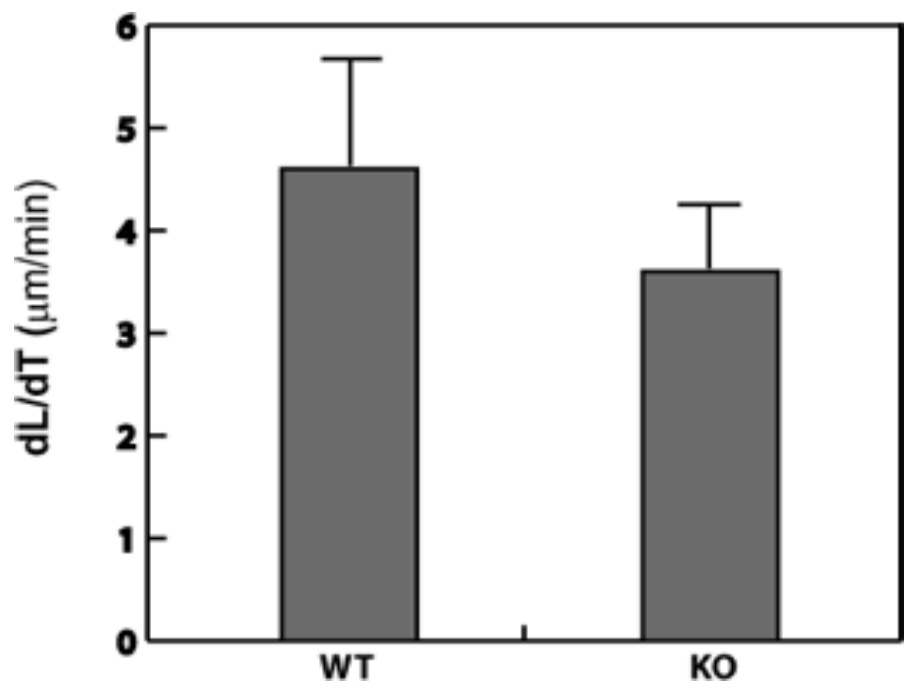

Figure 6 Impaired focal adhesion disassembly at cell trailing edges in Cav 1- MEFs. (B) Recoil velocity in MEFs. Each bar represent the mean \pm s.e.m. (error bars) from three replicate experiments. $P=0.3$ 


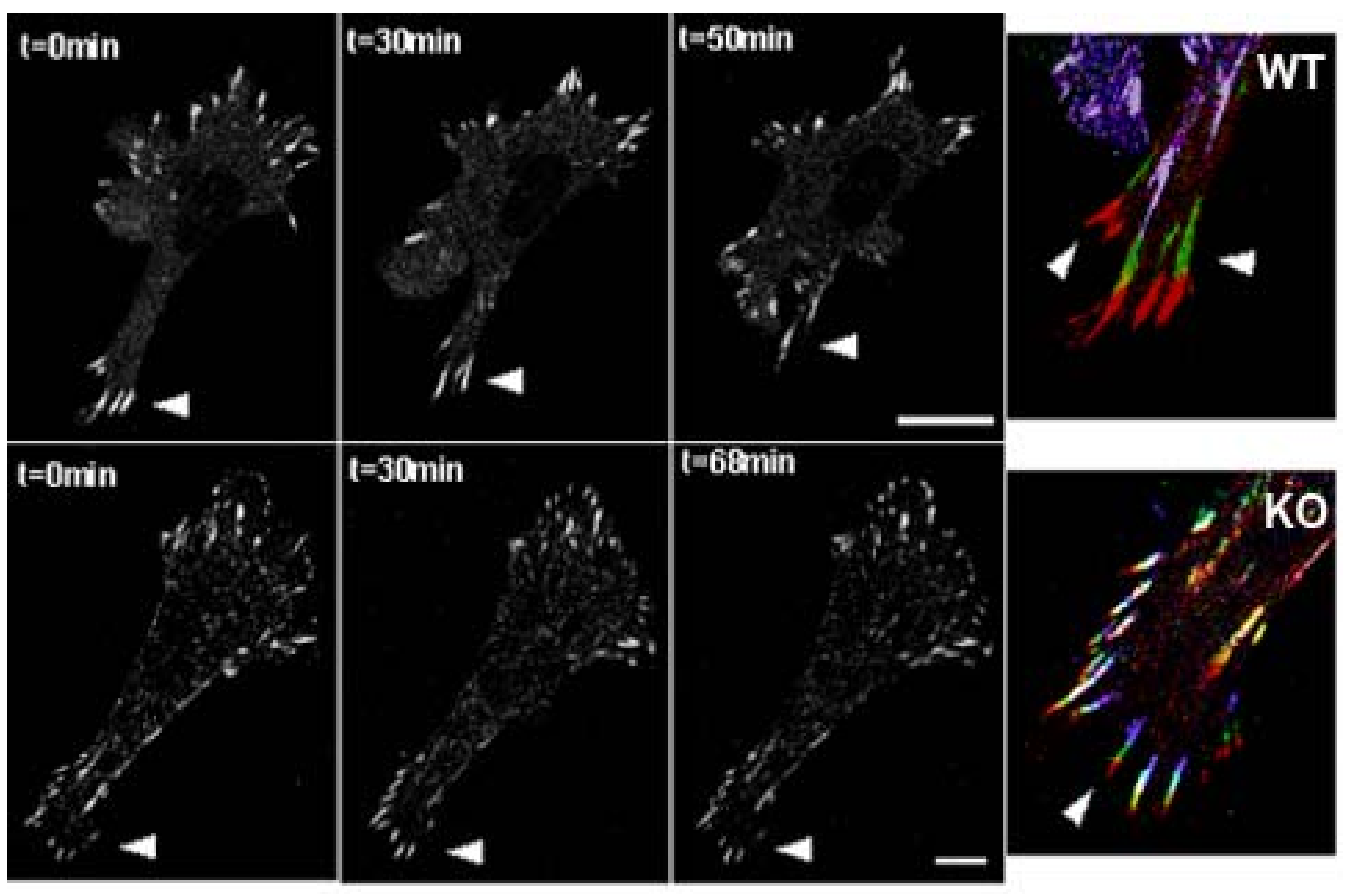

Figure 6 Impaired focal adhesion disassembly at cell trailing edges in Cav $^{-1-}$ MEFs. (c) Representative adhesion disassembly and tail recoil in $\mathrm{Cav}^{-1-}$ and wild-type MEFs. Sequential confocal images taken from a caveolin-1 null MEF demonstrate paxillin-containing trailing edge adhesions (arrowheads) predominantly remain stable for greater than one hour, while trailing edge adhesions in a wild-type MEFs have largely disassembled in the same time frame. Color overlays of the images demonstrate significant contact plasticity in the wild-type cell whereas only a few adhesions in the caveolin-1 null cell exhibit a modest degree of sliding (arrowheads). Scale bars, $20 \mu \mathrm{m}$. 


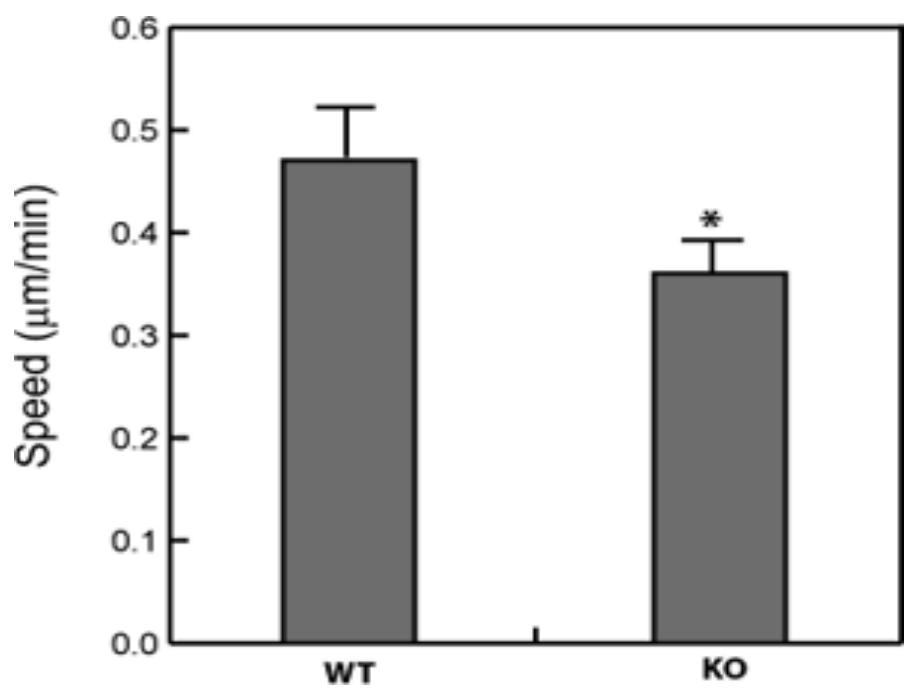

Figure 7 Analysis of chemokinetic motility in mutant and control cells. (a) Caveolin-1-deficient cells display a decrease in average scalar movement (speed) compared to control cells. Each bar represent the mean \pm SEM (error bars) from three replicate experiments. ${ }^{*}, P<0.01$ 


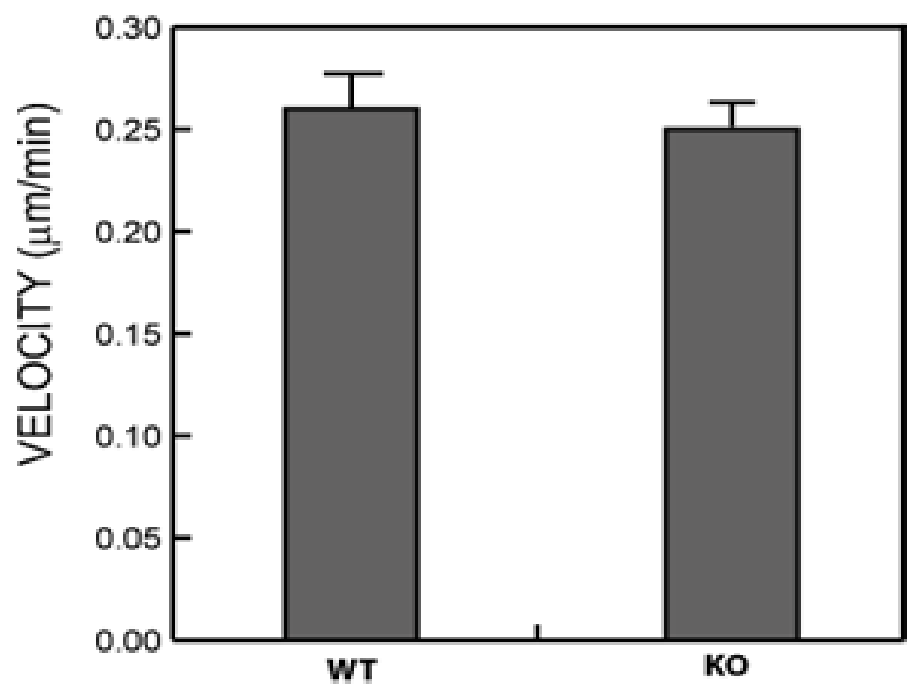

Figure 7 Analysis of chemokinetic motility in mutant and control cells. (b) No significant difference in average directional movement (velocity) was detected in mutant and control cells. Each bar represent the mean \pm SEM (error bars) from three replicate experiments. $P=0.8$ 


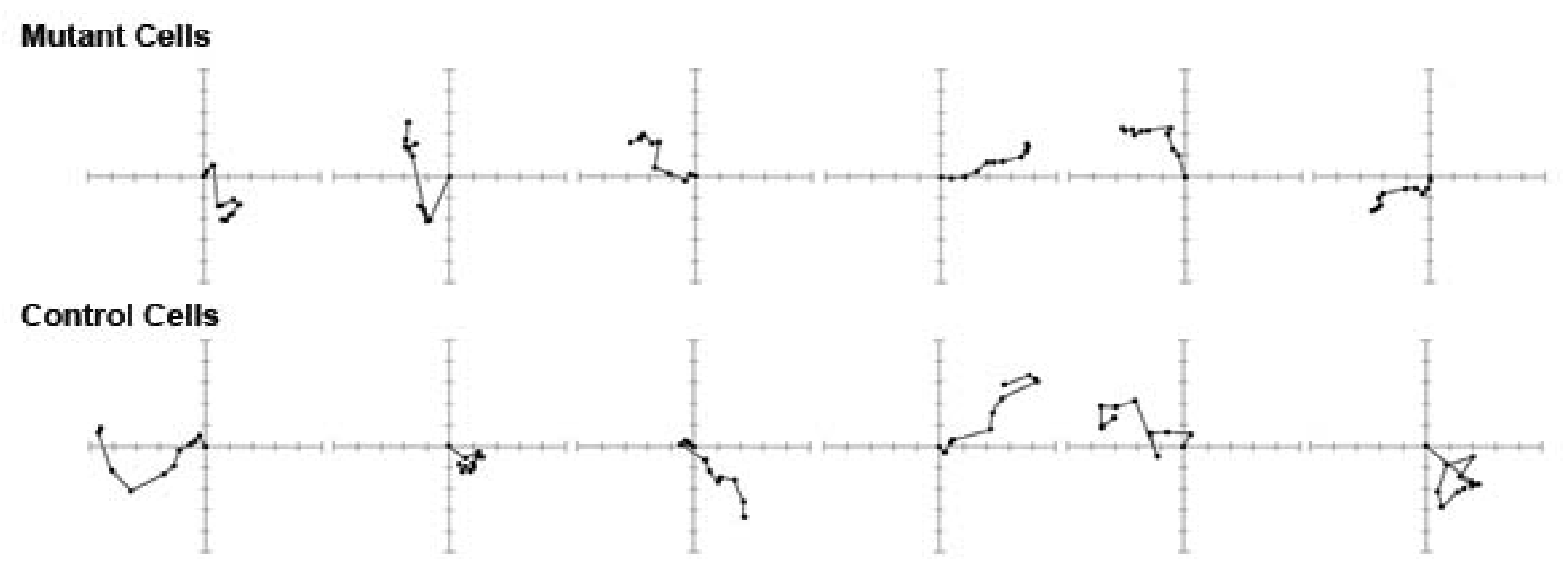

Figure 8 Directional Persistence is increased in caveolin-1 null MEFs (a) Migration paths of six representative pairs from 19 and 27 control and mutant cells are shown. Each point represents the position of the cell at 30 min intervals. Ten consecutive intervals are shown. Each axis tick represents a distance of $15 \mu \mathrm{m}$. 


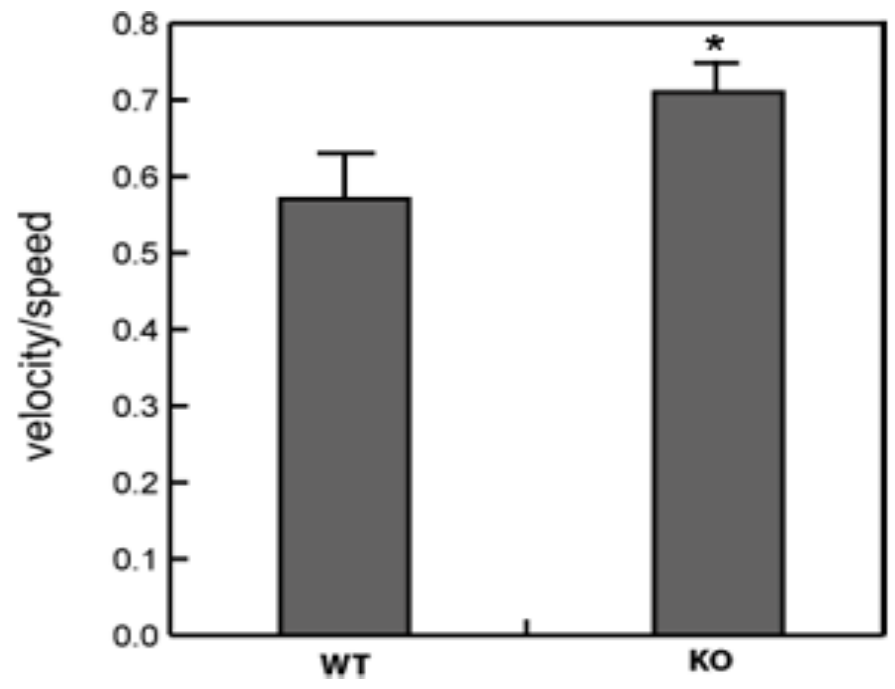

Figure 8 Directional Persistence is increased in caveolin-1 null MEFs

(b) Directonal persistence is defined as ratio of net movement (velocity) to total movement (speed). Caveolin ablation results a slight but significant increaseln directional persistence. Each bar represent the mean \pm SEM (error bars) from three replicate experiments. ${ }^{*}, P=0.02$ 


\section{REFERENCES}

Anderson, R. G. (1998). "The caveolae membrane system." Annu Rev Biochem 67: 199-225.

Ballestrem, C., B. Hinz, et al. (2001). "Marching at the front and dragging behind: differential alphaVbeta3-integrin turnover regulates focal adhesion behavior." J Cell Biol 155(7): 1319-32.

Beardsley, A., K. Fang, et al. (2005). "Loss of caveolin-1 polarity impedes endothelial cell polarization and directional movement." J Biol Chem 280(5): 3541-7.

Carragher, N. O., V. J. Fincham, et al. (2001). "Cleavage of focal adhesion kinase by different proteases during SRC-regulated transformation and apoptosis. Distinct roles for calpain and caspases." J Biol Chem 276(6): 4270-5.

Chen, H., D. M. Cohen, et al. (2005). "Spatial distribution and functional significance of activated vinculin in living cells." J Cell Biol 169(3): 459-70.

Chen, W. T. (1981). "Mechanism of retraction of the trailing edge during fibroblast movement." J Cell Biol 90(1): 187-200.

Cohen, A. W., R. Hnasko, et al. (2004). "Role of caveolae and caveolins in health and disease." Physiol Rev 84(4): 1341-79.

Ezratty, E. J., M. A. Partridge, et al. (2005). "Microtubule-induced focal adhesion disassembly is mediated by dynamin and focal adhesion kinase." Nat Cell Biol 7(6): 581-90.

Franco, S. J., M. A. Rodgers, et al. (2004). "Calpain-mediated proteolysis of talin regulates adhesion dynamics." Nat Cell Biol 6(10): 977-83.

Fujimoto, T., A. Miyawaki, et al. (1995). "Inositol 1,4,5-trisphosphate receptor-like protein in plasmalemmal caveolae is linked to actin filaments." J Cell Sci 108 ( Pt 1): 7-15.

Hommelgaard, A. M., K. Roepstorff, et al. (2005). "Caveolae: stable membrane domains with a potential for internalization." Traffic 6(9): 720-4.

Horwitz, A. R. and J. T. Parsons (1999). "Cell migration--movin' on." Science 286(5442): 1102-3.

Huang, C., Z. Rajfur, et al. (2003). "JNK phosphorylates paxillin and regulates cell migration." Nature 424(6945): 219-23. 
Isshiki, M., J. Ando, et al. (2002). "Sites of $\mathrm{Ca}(2+)$ wave initiation move with caveolae to the trailing edge of migrating cells." J Cell Sci 115(Pt 3): 47584.

Kaverina, I., O. Krylyshkina, et al. (1999). "Microtubule targeting of substrate contacts promotes their relaxation and dissociation." J Cell Biol 146(5): 1033-44.

Mayer, C., K. Maaser, et al. (2004). "Release of cell fragments by invading melanoma cells." Eur J Cell Biol 83(11-12): 709-15.

Nobes, C. D. and A. Hall (1995). "Rho, rac and cdc42 GTPases: regulators of actin structures, cell adhesion and motility." Biochem Soc Trans 23(3): 456-9.

Peters, K. R., W. W. Carley, et al. (1985). "Endothelial plasmalemmal vesicles have a characteristic striped bipolar surface structure." J Cell Biol 101(6): 2233-8.

Raftopoulou, M. and A. Hall (2004). "Cell migration: Rho GTPases lead the way." Dev Biol 265(1): 23-32.

Razani, B., J. A. Engelman, et al. (2001). "Caveolin-1 null mice are viable but show evidence of hyperproliferative and vascular abnormalities." J Biol Chem 276(41): 38121-38.

Ridley, A. J., M. A. Schwartz, et al. (2003). "Cell migration: integrating signals from front to back." Science 302(5651): 1704-9.

Rohlich, P. and A. C. Allison (1976). "Oriented pattern of membrane-associated vesicles in fibroblasts." J Ultrastruct Res 57(1): 94-103.

Rothberg, K. G., J. E. Heuser, et al. (1992). "Caveolin, a protein component of caveolae membrane coats." Cell 68(4): 673-82.

Rottner, K., A. Hall, et al. (1999). "Interplay between Rac and Rho in the control of substrate contact dynamics." Curr Biol 9(12): 640-8.

Shaul, P. W. and R. G. Anderson (1998). "Role of plasmalemmal caveolae in signal transduction." Am J Physiol 275(5 Pt 1): L843-51.

Smilenov, L. B., A. Mikhailov, et al. (1999). "Focal adhesion motility revealed in stationary fibroblasts." Science 286(5442): 1172-4. 
Stahlhut, M. and B. van Deurs (2000). "Identification of filamin as a novel ligand for caveolin-1: evidence for the organization of caveolin-1-associated membrane domains by the actin cytoskeleton." Mol Biol Cell 11(1): 325-37.

Stan, R. V. (2005). "Structure of caveolae." Biochim Biophys Acta 1746(3): 33448.

Thomsen, P., K. Roepstorff, et al. (2002). "Caveolae are highly immobile plasma membrane microdomains, which are not involved in constitutive endocytic trafficking." Mol Biol Cell 13(1): 238-50.

Webb, D. J., K. Donais, et al. (2004). "FAK-Src signalling through paxillin, ERK and MLCK regulates adhesion disassembly." Nat Cell Biol 6(2): 154-61.

Webb, D. J., J. T. Parsons, et al. (2002). "Adhesion assembly, disassembly and turnover in migrating cells -- over and over and over again." Nat Cell Biol 4(4): E97-100.

Williams, T. M. and M. P. Lisanti (2004). "The caveolin proteins." Genome Biol 5(3): 214 


\section{Study 3}

Characterization of Caveolin-1 Anchorage to Actin Stress Fibers at the Cell Trailing Edge 


\section{ABSTRACT}

Although well described, the association of caveolin-1 with the actin cytoskeleton remains uncharacterized. Specifically, the significance of caveolin in alignment with actin stress fibers at the cell perimeter has not been determined. Here we characterize this association in the context of cell migration. We show that caveolin-1 predictably associates with the cell perimeter depending on the direction of cell migration, and inhibition of cell contractility prevents caveolin polarization. Using live cell imaging in conjunction with photobleaching, we show that actin-associated caveolin-1 is extremely static upon polarization to the cell rear. In contrast, the initial polarization of caveolin-1 to retracting areas is highly dynamic. Furthermore, GM1 internalization at the cell rear is negligible, confirming that polarized caveolae are highly static. Forced disruption of the actin cytoskeleton by cytochalasin $D$ treatment results in caveolin-1 depolarization and disaggregation into small puncta displaying frenetic, kiss-andrun movement. Furthermore, cytoskeletal remodeling in response to change in direction of a cell results in similar caveolin depolarization. Taken together, our data indicate that rear polarized caveolin and caveolae do not function in constitutive endocytic rear-front recycling during cell migration. We conclude that polarized caveolin might function to tether the cell membrane to the actin cytoskeleton, or that such polarization might be vestigial, a consequence of trailing edge recruitment of caveolin-1 prior to polarization. 


\section{INTRODUCTION}

The arrangement of caveolae in longitudinal arrays in alignment with actin stress fibers at the plasma membrane has long been appreciated (Stan 2005). Caveolin-1, the principle protein component of caveolae, has similarly been detected in such linear arrays, as well as discrete plasma membrane domains originally thought to be situated at the leading edge of the cell (Rothberg, Heuser et al. 1992). The interaction of caveolin-1 with actin stress fibers in discrete patches at the cell perimeter is thought to be mediated by direct interaction of caveolin with filamin, an actin cross-linking protein (Stahlhut and van Deurs 2000).

A flurry of reports using caveolin-1-GFP chimeras in conjunction with live cell imaging have demonstrated caveolin is immobilized at the cell membrane/perimeter by interaction with core or cortical actin fibers. Mundy et al. demonstrated that depolymerization of the actin cytoskeleton triggered rapid egress of caveolin-GFP from the cell surface towards the centrosomal region in CHO cells (Mundy, Machleidt et al. 2002). Moreover, FRAP/FLIP experiments of caveolin-1-GFP concentrated at the cell perimeter demonstrated that GFPlabeled caveolae were highly immobile at the cell surface in HeLA, A431, and MDCK cells (Thomsen, Roepstorff et al. 2002); complimentary total internal reflection fluorescence microscopy experiments of caveolin-1-GFP positive spots demonstrated that the protein is largely static in the ventral plasma membrane of

unstimulated HeLa cells (Tagawa, Mezzacasa et al. 2005). An emergent dogma is thus that caveolae in unstimulated cells are not constitutively endocytic, but 
stabilized at the cell surface, where the membranous organelles are needed to serve a multiplicity of functions (Hommelgaard, Roepstorff et al. 2005).

We and others have demonstrated the polarization of caveolin-1 and caveolae at the rear of migrating human umbilical vein and bovine aortic endothelial cells (Isshiki, Ando et al. 2002; Parat, Anand-Apte et al. 2003; Beardsley, Fang et al. 2005) in association with core actin stress fibers. We have recently demonstrated by live cell imaging that initial association of caveolin-1 with the distal tips of actin stress fibers terminating at focal adhesions predisposes adhesion disassembly and collapse of radially-oriented stress fibers into a large bundle at the cell rear, co-aligned with caveolin-1, and oriented perpendicularly to the direction of cell movement. Currently it is not known whether caveolin polarized to the cell rear is immobilized by actin, in analogy to other perimeter-associated concentrations of the protein.

Herein, we characterize the contribution of the actin cytoskeleton to caveolin polarization in the context of endothelial cell migration. In agreement with previous studies, we found that caveolin/caveolae associated with actin stress fibers situated at the cell rear are highly immobile. Moreover, we observed that acto-myosin tension is important for the initial recruitment and polarization of caveolin-1 to the cell rear, but not in the maintenance of such polarity. Finally, through cytoskeletal disassembly in conjunction with live cell imaging, we observed a putative subdomain of the actin cytoskeleton, polarized in bundles at the cell rear in association with caveolin, which may shed light on the function of this unique cytoskeletal entity in cell migration. 
We conclude that polarized caveolae are remarkably stable at the cell rear, arguing against a role for the organelle in constitutive rear-to-front membrane or cytoskeletal/adhesion protein recycling during migration. The observation that caveolin-1 recruitment and interaction with actin at the cell perimeter is predictably associated with cell movement leads us to conclude that, at least in endothelial cells, the presence of caveolin/caveolae are not simply sequestered at the cell perimeter by the actin cytoskeleton to perform various other functions; rather, that caveolin is actively involved in cytoskeletal rearrangement (e.g. adhesion breakdown and subsequent actin reorientation) that takes place during motility. 


\section{EXPERIMENTAL PROCEDURES}

Materials: Reagents and other supplies were obtained from the following commercial sources: Antibodies against caveolin and GFP were purchased from Santa Cruz Biotechnology (Santa Cruz, CA). Goat serum, FITC and Rhodamine Red-X conjugated secondary antibodies were obtained from Jackson ImmunoResearch Laboratories (West Grove, PA). CTB-TRITC was purchased from Molecular Probes (Eugene, Oregon). Blebbistatin was purchase from Calbiochem (San Diego, CA). The bicinchoninic acid (BCA) protein assay kit was purchased from Pierce (Rockford, IL). The full-length cDNA encoding caveolin-1 was fused in-frame to the N-terminus of GFP. EcoRI and BamHI restriction sites were added to 5' and 3' ends of murine caveolin-1 cDNA by polymerase chain reaction (PCR) using TripleMaster® PCR System (Brinkmann instruments, Inc.). The PCR products were subcloned into the EcoRI and BamHI sites of pEGFP-N1. pmRFP-N1 was generated by replacing GFP of pEGFP-N1 with mRFP (mRFPwGBD was provided by W.M. Bement, University of Wisconsin-Madison, Madison, WI). Caveolin-1-RFP was constructed by subcloning the murine caveolin-1 cDNA into pmRFP-N1. pAcGFP1-Actin vector was purchased from BD Biosciences.

Cell culture and transfection. Primary cultured endothelial cells from the human umbilical vein (HUVECs) were obtained as described previously from consenting healthy, term patients according to institutional guidelines (Beardsley, Fang et al. 2005). HUVECs were grown in MCDB supplemented with $20 \%$ heatinactivated newborn calf serum, $5 \%$ heat-inactivated human serum and 
endothelial cell growth supplement. Transient transfections of HUVECs and MEFs were performed using Lipofectin and Lipofectamine reagents (Life Technologies, Inc.), respectively, according to the protocol provided by the manufacturer. Cells were analyzed $36-48 \mathrm{~h}$ after transfection.

Microscopy and analysis. Cells expressing caveolin-1-GFP or coexpressing caveolin-RFP and paxillin-GFP were incubated on Lab-Tek II 2-well chambered coverglass. A temperature controlled heating stage, $\mathrm{CO}_{2}$ controlled incubator insert and Zeiss objective heater were used to maintain the cells at $37^{\circ}$ $\mathrm{C}$ and $5 \% \mathrm{CO}_{2}$. Images were captured using a Zeiss LSM 510 laser scanning confocal system, with an Axiovert 200M inverted microscope, 60x or 40x oil immersion lenses, and Argon and HeNe lasers. Fluorescent and/or DIC images were captured in 2-300 second intervals using Zeiss time-lapse software. Image analysis was performed using LSM510 software and Clmaging software (Compix Inc., Cranberry, PA). CTB-TRITC labeling was performed as described (McIntosh and Schnitzer 1999) with the following modifications: cells were incubated with $1 \mu \mathrm{g} / \mathrm{ml}$ CTB-TRITC in whole medium for 15 minutes at $4^{\circ} \mathrm{C}$. The cells were washed twice and allowed to recover for 10 minutes. The cells were then analyzed by live cell imaging.

Immunocytochemistry. Endothelial cells were fixed with $2 \%$ paraformaldehyde for 20 min, permeabilized with $1 \%$ Triton X-100, blocked with $5 \%$ goat serum, and stained with specific antibody against caveolin-1 or other signaling molecules. Bound primary antibodies were detected using fluorescein- 
or rhodamine-conjugated secondary antibodies. The immunostained cells were mounted in the presence of Slow-Fade reagent. Immunostaining was visualized and photographed using a Zeiss LSM 510 confocal microscope.

Blebbistatin Treatment. To determine the effect of blebbistatin on caveolin-1 polarization, HUVECs were seeded overnight in a six well dish (90000 cells/well). The cells were washed once in $1 \%$ newborn calf serum followed by addition of $30 \mu \mathrm{M}$ blebbistatin or vehicle (DMSO) for $30 \mathrm{~min}$. The cells were then harvested and seeded on fibronectin-coated coverslips for varying times. The cells were then fixed and immunostained for caveolin. 12 low power fields were analyzed for each time point/treatment. The results are representative of 2 independent experiments performed in duplicate. Blebbistatin treatment of polarized cells was performed similarly with the following modifications. Cells were seeded on fiberonectin-coated coverslips and incubated overnight to allow maximum caveolin-1 polarization. The cells were washed once and incubated with $30 \mu \mathrm{M}$ blebbistatin or vehicle (DMSO) for different times. The results are representative of 1 experiment performed in duplicate.

Fluorescence Recovery After Photobleaching (FRAP) analysis. HUVECs were transfected with $2 \mu \mathrm{g}$ Caveolin-1-GFP or empty GFP vector per six well dish. $24 \mathrm{~h}$ post transfection, HUVECs were seeded at subconfluency on LabTek chambered coverglass and incubated overnight to allow for migration. Cells in which caveolin-1-GFP appeared distinctly polarized at the rear were chosen for analysis. A given region was bleached at full laser power (100\% power, 100\% transmission, 50 iterations) using the $488 \mathrm{~nm}$ line from a $30 \mathrm{~mW}$ Argon/2 laser. 
Recovery of fluorescence was monitored by scanning the region at low laser power.

Images were processed using Image $\mathrm{J}$ (http://rsb.info.nih.gov/ij/). Quantification of FRAP experiments was performed as described (Tagawa, Mezzacasa et al. 2005), by measuring the fluorescence intensity of the whole cell and of the bleached area before, directly after, and during recovery of bleaching. To correct for overall bleaching, the relative intensity of fluorescence (RI) was calculated according to the following equation: $\mathrm{RI}=\left(\mathrm{I}_{\text {total[0] }]} / \mathrm{I}_{\text {bleached[0] }}\right) \mathrm{x}$ $\left(I_{\text {bleached[t] }} I_{\text {total[t] }}\right)$, where $I_{\text {total(0) }}$ is the total intensity of the cell before bleaching, $I_{\text {bleached(0) }}$ the total intensity of the bleached area before bleaching, $I_{\text {bleached(t) }}$ the intensity of the bleached area at time $t$, and $I_{\text {total(t) }}$ the intensity of the whole cell at timet. 


\section{RESULTS}

Localization of caveolin-1 at the cell perimeter is apparent on immunofluorescent labeling (Dupree, Parton et al. 1993; Scherer, Okamoto et al. 1996; Oh and Schnitzer 2001; Mundy, Machleidt et al. 2002), but of undetermined significance. To relate perimeter-association of the molecule to cell movement, we analyzed caveolin-1-GFP in a moving HUVEC for over two hours (Figure $1 A$ ). In this time frame, the cell changed direction three times. Coincident with direction change, caveolin-1 appeared polarized each time at the nascent cell rear. Between direction changes, caveolin-1 initially remains perimeter-associated, but eventually repolarizes as the cell retracts in a new direction. Thus, caveolin localization at the cell perimeter predictably occurs in relation to cell movement.

To verify this result, we labeled the resident caveolar sphingolipid, GM1, with cholera toxin subunit B conjugated with TRITC in migrating HUVECs. Similar to caveolin-1 signal, CTB-TRITC distinctly appeared as a stripe at the cell rear (Figure 1B). Moreover, the CTB-TRITC signal predictably appeared at the cell perimeter as the cell changed direction. Thus caveolin and caveolar association with the cell perimeter are linked to cell movement.

We previously reported that caveolin-1 is recruited to trailing edge focal adhesions, whereupon it initiates adhesion disassembly and trailing edge and actin stress fiber collapse. We postulated that strain induced by acto-myosin contraction was responsible for caveolin-1 recruitment and ultimately polarization 
at the cell rear. To test this hypothesis, we used the selective non-muscle myosin inhibitor, blebbistatin, to diminish cell contractility, and subsequently monitored caveolin-1 polarization. When cells were pretreated with $30 \mu \mathrm{M}$ blebbistatin, harvested, and seeded on fibronectin-coated coverslips in blebbistatin-containing medium, caveolin-1 polarization was reduced nearly 2.5 fold (Figure $2 A, B$ ). In contrast, blebbistatin treatment has no effect caveolin-1 that had previously polarized (Figure 2C). Cells were incubated on fibronectin overnight, allowing time for maximal caveolin-1 polarization, and then treated with blebbistatin for varying times. No significant difference was seen between blebbistatin- and vehicle-treated cells. Thus, initial, but not sustained caveolin-1 polarization depends on cell contractility.

Association of caveolin-1 in co-alignment with the actin cytoskeleton at the cell perimeter has been described (Stahlhut and van Deurs 2000). We have also shown such co-alignment at the cell rear. To explore this relationship, we cotransfected HUVECs with caveolin-1-GFP and actin-RFP and then analyzed the cells by live cell imaging. Indeed co-alignment of caveolin-1 and actin stress fibers occurred at the cell rear during migration (Figure $3 A$ ). Moreover, this coalignment is stable, persisting for greater than $50 \mathrm{~min}$ in the example shown (Figure 3B). Importantly, the cell shown moves in the same direction over the 50 min period. Thus, stable co-alignment of caveolin and actin stress fibers occurs at the cell rear. 
Recent photobleaching and TIR-FM studies have indicated that surface caveolin-1 is highly stable (Tagawa, Mezzacasa et al. 2005). Although perhaps anticipated, it is not currently known whether polarized caveolin-1 in migrating cells is stable or not. Indeed, Parat et al. have speculated that caveolin-1 at the rear may be involved in constitutive membrane recycling during migration (Navarro, Anand-Apte et al. 2004), implicitly suggesting that rear-associated caveolin is highly dynamic. To resolve this point, we studied FRAP of polarized caveolin-1-GFP in migrating cells.

Bleaching of polarized caveolin-1-GFP in posteromedial areas of the cell (i.e. away from the baso-lateral corners) resulted in negligible FRAP over extended time periods (Figure $4 A, B$ ). This result strongly suggests that, beyond co-localization with actin stress fibers, caveolin-1 is highly immobilized on the core actin cytoskeleton at the rear of migrating cells. Moreover, the static nature of caveolin-1 seems to preclude a role in membrane trafficking, at least in the context of motility.

Interestingly, analysis of basolateral areas initially devoid of caveolin signal, but proximal to polarized caveolin-GFP, revealed an increase in fluorescence intensity upon recoil of such areas (Figure 5a). Recoil of the basolateral corners resulted in extension of extant caveolin polarization. Analysis of fluorescence intensity of one such event is shown in Figure $5 b$. In contrast to the highly immobile caveolin signal in postero-medial areas, the fluorescent intensity of caveolin-1 sharply increases in retracting cell corners. 
Thus, caveolin-1 is highly dynamic in its recruitment to retracting areas, but subsequently immobilized on actin stress fibers.

To verify the immobility of caveolar domains at the cell rear, migrating HUVECs were incubated with CTB-TRITC, washed and analyzed by live cell imaging. Monitoring the TRITC signal simulates a pulse chase experiment-thus, if GM1 is rapidly cleared from cell perimeter, as would be expected in caveolar/lipid raft recycling during migration, the pulse of CTB-TRITC would correspondingly be cleared. CTB-TRITC polarized at the cell rear of migrating HUVECs was analyzed (Figure 6, see also Figure 1B). Strikingly, CTB signal remained stably polarized (Figure 6) as long as the cell moved persistently in one direction, verifying the immobility of caveolae at the cell rear during migration.

The destabilization of surface-associated caveolin has been demonstrated by cytoskeletal disruption and treatment with simian virus 40 (SV40) (Pelkmans, Puntener et al. 2002). To determine whether such treatment analogously destabilizes caveolin at the rear, we treated caveolin-GFP expressing HUVECs with a low dose $(25 \mathrm{~nm})$ of cytochalasin $\mathrm{D}$ and observed the effects by live cell imaging. Two representative cells are shown. In the first example (Figure 7A), treatment results in the gradual peeling of caveolin-1 GFP away from the cell rear. Eventually an entire rod-like strip of caveolin signal separates from the rear, violently collapsing towards the front of the cell. Interestingly, caveolin still appears as a stripe, albeit reduced in size. The organized shape of caveolin signal is presumably due to residual association with fragmented actin stress 
fibers that have passively recoiled to the cell front. A second example demonstrates snapping of polarized caveolin-1 towards a trailing fiber upon cytochalasin treatment (Figure 7B). Significantly, residual polarized caveolinGFP remains in both examples gradually disaggregating and displaying frenzied local movement.

The integrity of the actin cytoskeleton is thus a critical factor in caveolin polarization. How does caveolin-1 depolarize in an intact, untreated cell? On observation of caveolin-1-GFP in motile cells, we noticed that lamellipodia sometimes bloom out of areas of caveolin-1 polarization (Figure $8 A$ ), initiating a change in direction. Lamellipodial blooming is coincident with stress fiber disassembly and depolarization of caveolin-1-GFP signal, which disaggregates into puncta displaying frenzied local movement similar to that described by Pelkmans (Pelkmans and Zerial 2005)(Figure 8B). Despite depolarization and local movement, the caveolin-GFP signal does not appear to internalize to a caveosomal compartment, but remains in the same vicinity for at least 30 minutes after initial disaggregation. Importantly, no new cell retractions occur in this time frame. 


\section{DISCUSSION}

We have shown that caveolin-1 association with the core actin cytoskeleton at the cell perimeter occurs predictably with cell movement. Since caveolin-1 is reported to function in myriad ways at the cell surface (Anderson 1993; Travis 1993; Hecker, Mulsch et al. 1994; Lisanti, Scherer et al. 1994; Lisanti, Tang et al. 1994; Anderson, Chen et al. 1996; Lisanti, Li et al. 1996; Vey, Pilkuhn et al. 1996; Couet, Sargiacomo et al. 1997; Anderson 1998), such perimeter localization might be tantalizing verification of a given function of caveolin at the cell surface; however, the influence of endothelial cell migration on caveolin-1 localization must be taken into account even if motility is not being studied. Furthermore, the dramatic and predictive effect of cell movement on caveolin-1 localization may suggest that caveolin-1 does not serve myriad functions after all, but rather acts specifically in cytoskeletal rearrangement in vitro. Extrapolation of this claim in vivo is problematic, given eNOS dysfunction present in caveolin null animals and the clearly established role of caveolin as an eNOS inhibitor (Sessa 2005)); however, deficits in vascular remodeling and angiogenesis (Woodman, Ashton et al. 2003; Sonveaux, Martinive et al. 2004; Yu, Bergaya et al. 2006) could well be explained by a putative role for caveolin-1 in cytoskeletal remodeling.

We show by live cell imaging, photobleaching, and a lack of CTB turnover, that caveolin-1 stably associates with bundles of actin stress fibers at the cell rear. Interestingly, caveolin-1 does not co-align with stress fibers in any other part of the cell. This disparity suggests that caveolin-1 is selectively 
recruited to areas of high traction, resulting in focal adhesion disassembly, retraction, and subsequent cell polarization. The concentration of multiple individual stress fibers from inside the cell to a single point (i.e. focal adhesion) at the cell perimeter could explain why caveolin is not recruited internally (see Diagram 1). Furthermore, the stability of caveolin at the cell rear precludes its role in constitutive endocytosis during cell motility, consistent with reports in other cell types (Hommelgaard, Roepstorff et al. 2005). Thus recycling of membrane or cytoskeletal components does not seems to be a priniciple function of caveolin during cell motility.

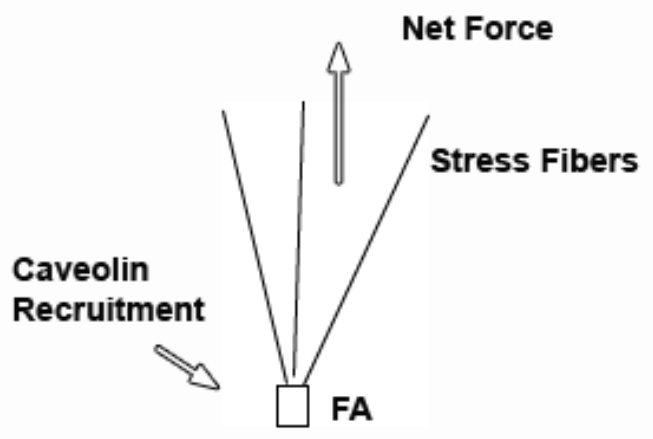

Diagram 1

The immobility of polarized caveolin-1 stands in contrast to the dynamic recruitment of caveolin prior to polarization. This is in agreement with data by Mundy et al. in which caveolin-1 is dynamically shuttled by microtubules to the cell surface, whereupon it is immobilized by cortical actin (Mundy, Machleidt et al. 2002). It is interesting to speculate that caveolin recruitment to the cell perimeter during migration is also mediated by microtubules, which are known to target 
focal adhesions in the retracting edge (Kaverina, Krylyshkina et al. 1999; Ezratty, Partridge et al. 2005). Indeed, Kaverina et al. suggested that microtubule targeting to focal adhesions may deliver a "relaxing factor" that disassembles the adhesions (Kaverina, Krylyshkina et al. 1999). It is interesting to speculate that caveolin-1 may be one such factor by which microtubule targeting affects adhesion breakdown, although such an assertion is premature at this time.

Lastly, we show that cytoskeletal disruption results in shrinkage and displacement of polarized caveolin en masse to other regions of the cell. This result suggests that polarized actin oriented normal to the direction of movement is highly prestressed, and may act as a lever on which the rest of the actin cytoskeleton can focus tension. Moreover, as seen by the two examples herein, this function seems to oscillate between trailing and leading edges. Thus, we demonstrate a putative role for caveolin-1-associated actin at the cell rear. Indeed, caveolin-associated actin may be considered a subdivision of the core actin cytoskeleton, representing a level of complexity to the cytoskeleton not anticipated by Ingber's cell tensegrity model (Ingber 1997).

Interestingly, caveolin-GFP remained associated with actin after fragmentation, violently pulled toward the cell front or trailing fiber see Figure ?). This is consistent with our results that inhibition of myosin by blebbistatin affects caveolin polarization before, but not after such polarization; moreover, such residual association of caveolin-1 with stress fiber fragments edifies the stability of this interaction. Given the catastrophic events that follow actin disassembly by 
cytochalasin treatment, such disassembly must be carefully and locally coordinated on reversal of direction. The contribution of the nascent lamellipodium to load bearing and stress fiber disassembly was not appreciated in this study, but further investigation is certainly warranted. Indeed, this rear-tofront transition may be exploited to gain insight the temporal function of several molecules involved in lamellipodial formation as well as actin-fiber destabilizing proteins.

In summary, we show for the first time that caveolin-1 predictably and stably associates with the actin-cytoskeleton at the cell perimeter of migrating cells. Moreover, we demonstrate that acto-myosin tension is prerequisite for caveolin polarization, but is not necessary for sustained polarization. Lastly, we demonstrate that actin depolymerization, either pharmacological or as a consequence of reversal of cell movement, results in disaggregation of polarized caveolin-1. We suggest that caveolin-1 polarization may be a vestigial result of its function in adhesion turnover, or alternatively serve to tether the plasma membrane to the core actin cytoskeleton. 

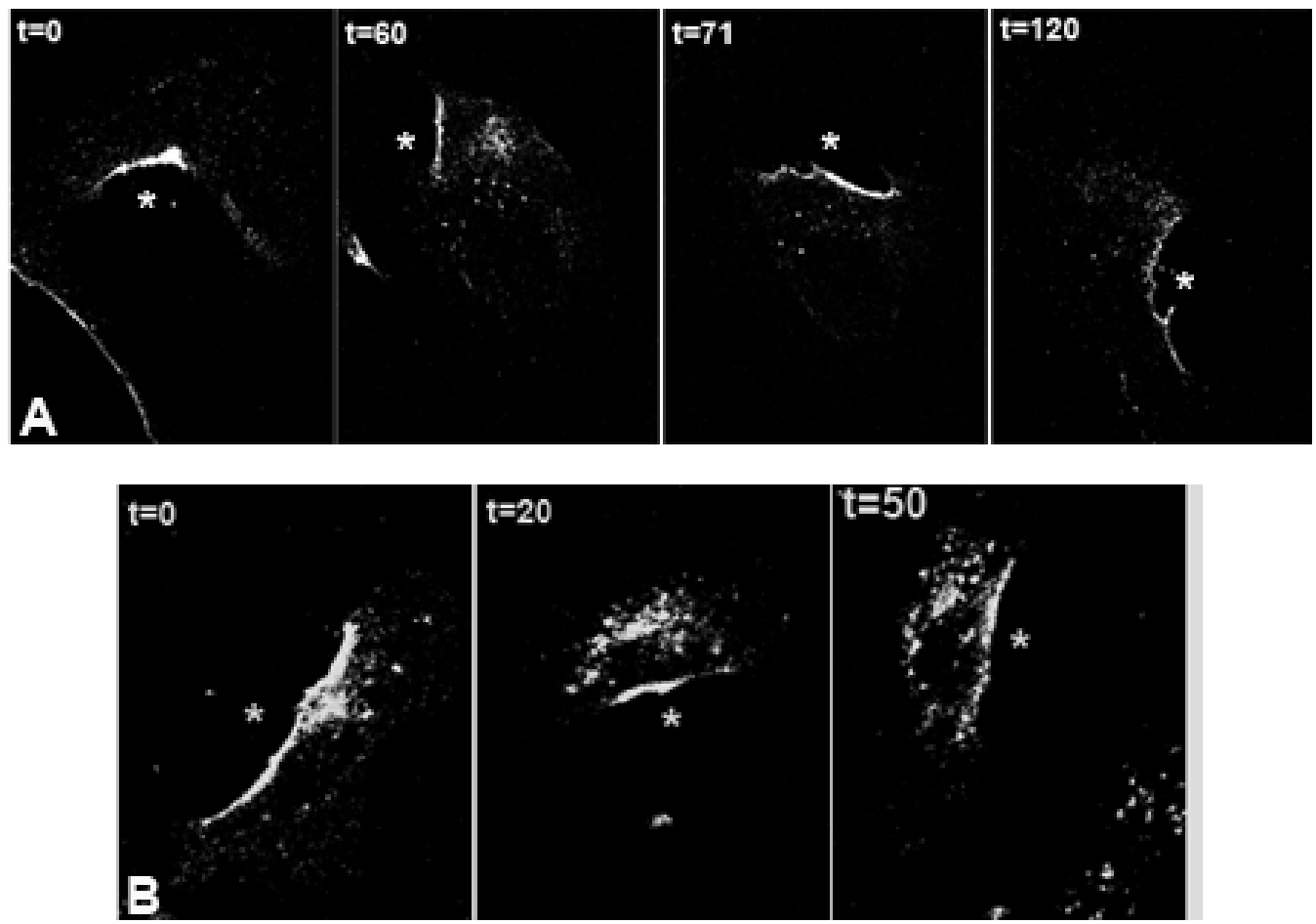

Figure 1 Caveolin-1 and CTB-TRITC accumulation at the cell perimeter occurs predictably with cell movement. (A) A time series of a caveolin-GFPexpressing, motile HUVEC reveals that caveolin-1 association with the cell perimeter occurs predictably with cell movement. In the first hour, the cell changes direction from 12 to 3 o'clock. The orientation of caveolin accordingly changes (asterisk). Two more right angle shifts occur in the 60-71 and 71-120 time intervals. Again, perimeter-associated caveolin-1 signal reflects the current direction of the cell. (B) GM1, a resident caveolae lipid, also predictably associates with the cell perimeter in relation to cell movement, as evidenced by CTB-TRITC labeling (asterisks). 


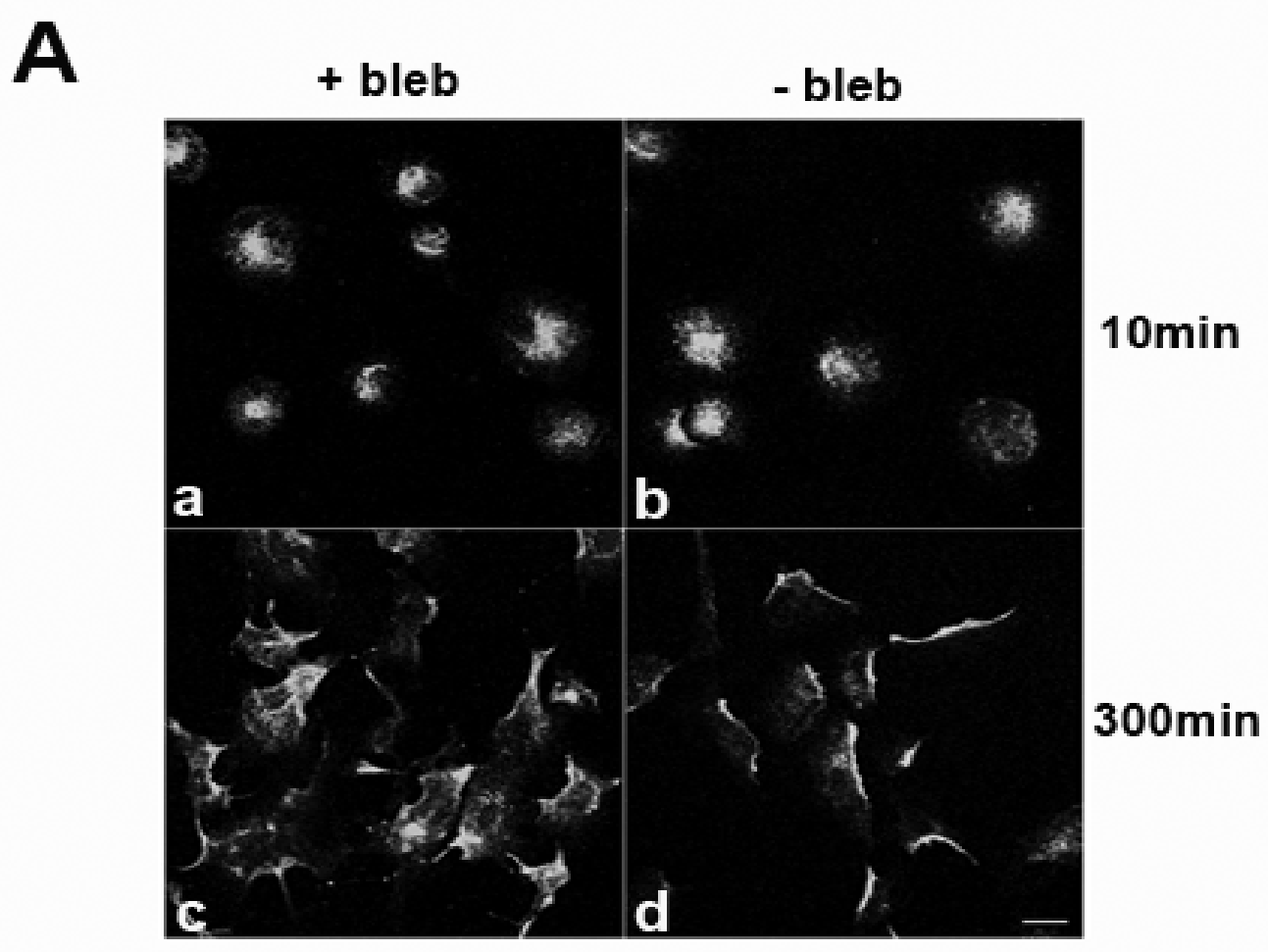

Figure 2 Initial recruitment of caveolin-1 to the cell perimeter is dependent on cell contractility. (A) HUVECs preincubated with $30 \mu \mathrm{M}$ blebbistatin to inhibit myosin II were seeded and allowed to spread on fibronectin coated coverslips. Initial attachment and spreading were not affected by blebbistatin treatment, (a). Subsequent polarization of caveolin-1 was reduced in blebbistaintreated cells (c) compared to mock-treated cells (d). Scale bar, $20 \mu \mathrm{m}$. 
B

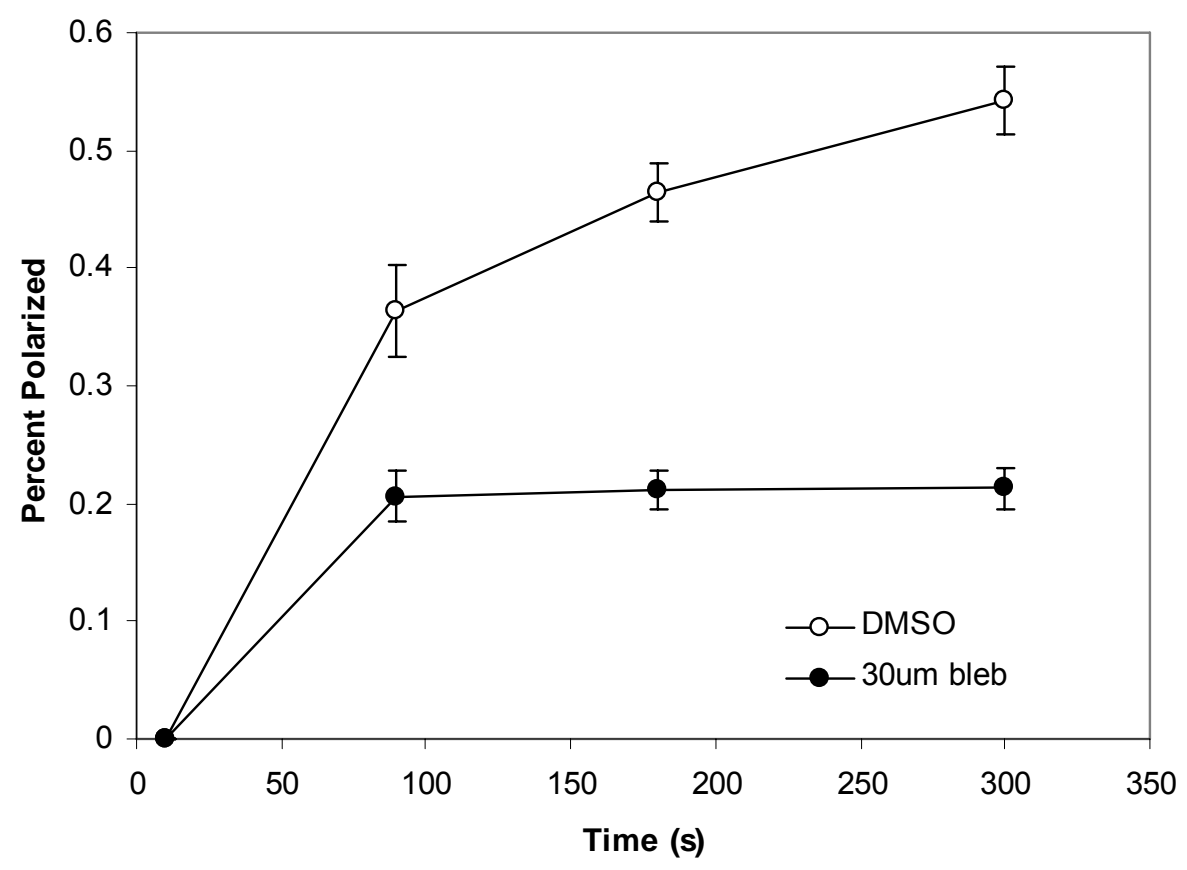

Figure 2 Initial recruitment of caveolin-1 to the cell perimeter is dependent on cell contractility. (B) Average ( \pm s.e.m.) fraction of caveolin-polarization in control- and blebbistatin-treated cells over time. Blebbistatin resulted in a $\sim 2.5$ fold reduction in caveolin polarization after $5 \mathrm{~h}$ on fibronectin. The results are representative of two separate experiments. 


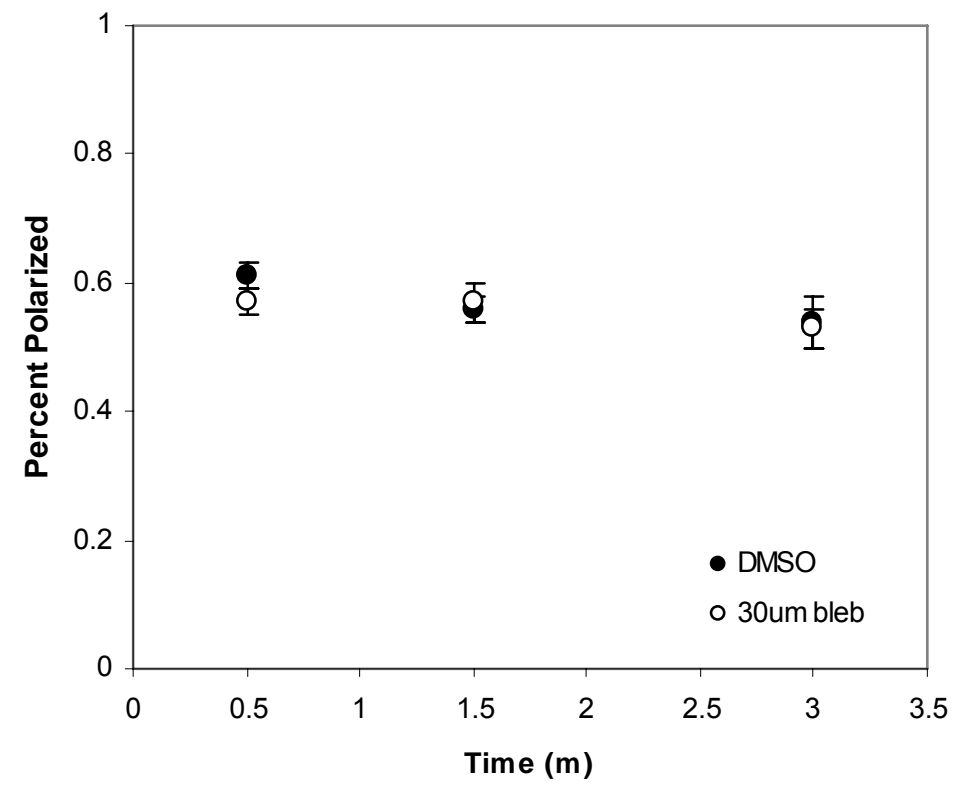

Figure 2 Sustained polarization of caveolin-1 to the cell perimeter is not dependent on cell contractility. (C) Average ( \pm s.e.m.) fraction of caveolinpolarization in control- and blebbistatin-treated cells over time. Blebbistatin treatment did not have a significant effect on caveolin-1 that had already polarized. 


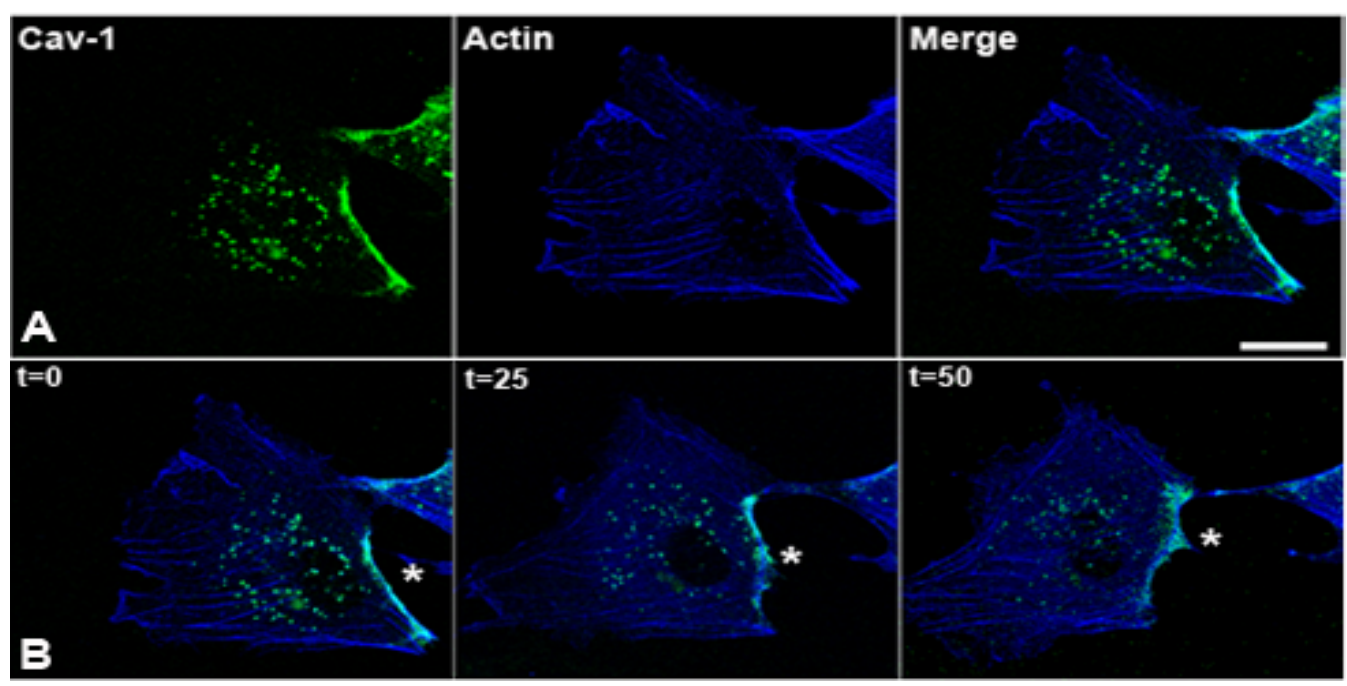

Figure 3 Caveolin-1 and actin stress fibers stably co-align at the cell rear. (A) A migrating HUVEC coexpressing caveolin-1-GFP (green) and actin-RFP (blue). Note co-alignment of caveolin-1 with collapsed actin stress fibers at the cell rear. Note also lack of alignment of caveolin signal with the rest of the actin cytoskeleton. Scale bar, $20 \mu \mathrm{m}$. (B) A time series of the same cell demonstrates that the co-alignment of caveolin with stress fibers is quite stable (asterisks) when migration persists in the same direction. 


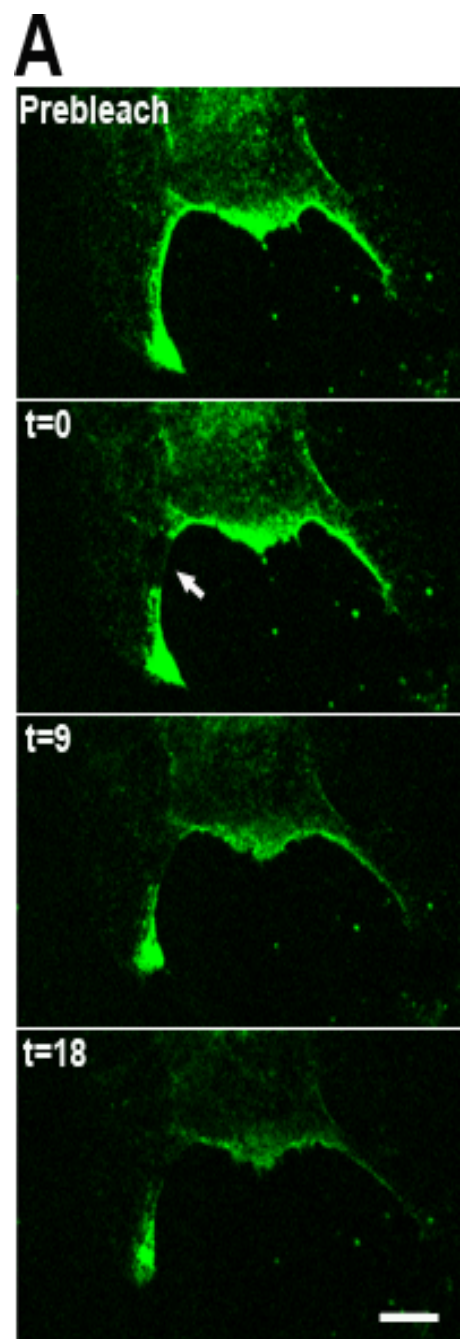

Figure 4 Photobleaching analysis of polarized caveolin-1. (a) An inward region of polarized caveolin-1-GFP in a HUVEC is shown before, immediately after, 9 , and 18 minutes post bleaching. The bleached area is denoted (arrow, 0 $\mathrm{min}$ ). Please note that the bleached area shows little fluorescence recovery (Bar, $10 \mu \mathrm{m})$. 
B

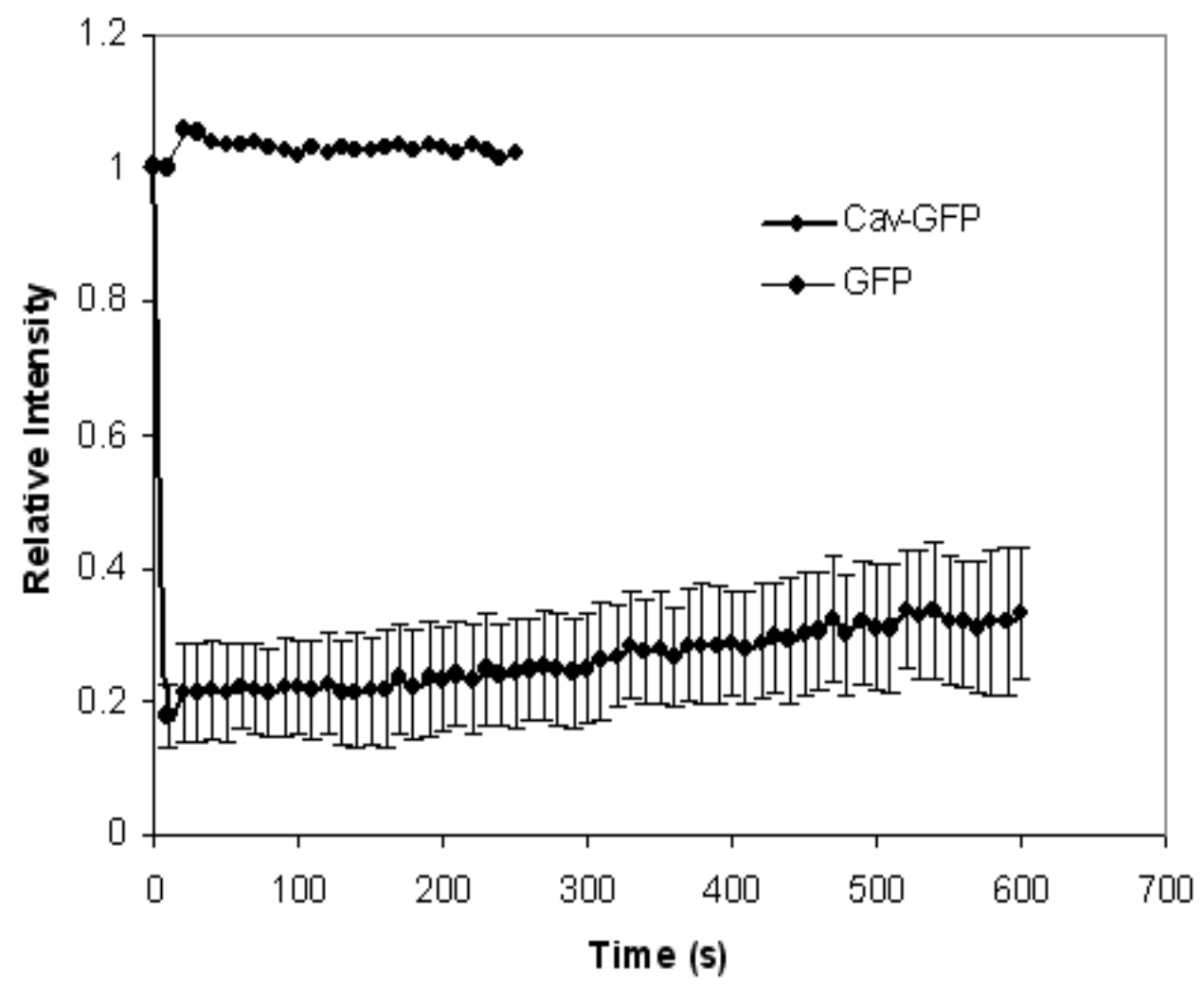

Figure 4 Photobleaching analysis of polarized caveolin-1. (b) Analysis of Caveolin-1-GFP FRAP. Fluorescence intensity of bleached areas were recorded every ten seconds for ten minutes. Error bars represent the s.e.m. of three independent experiments. An example of GFP FRAP, which occurs within a second, is shown for comparison. 


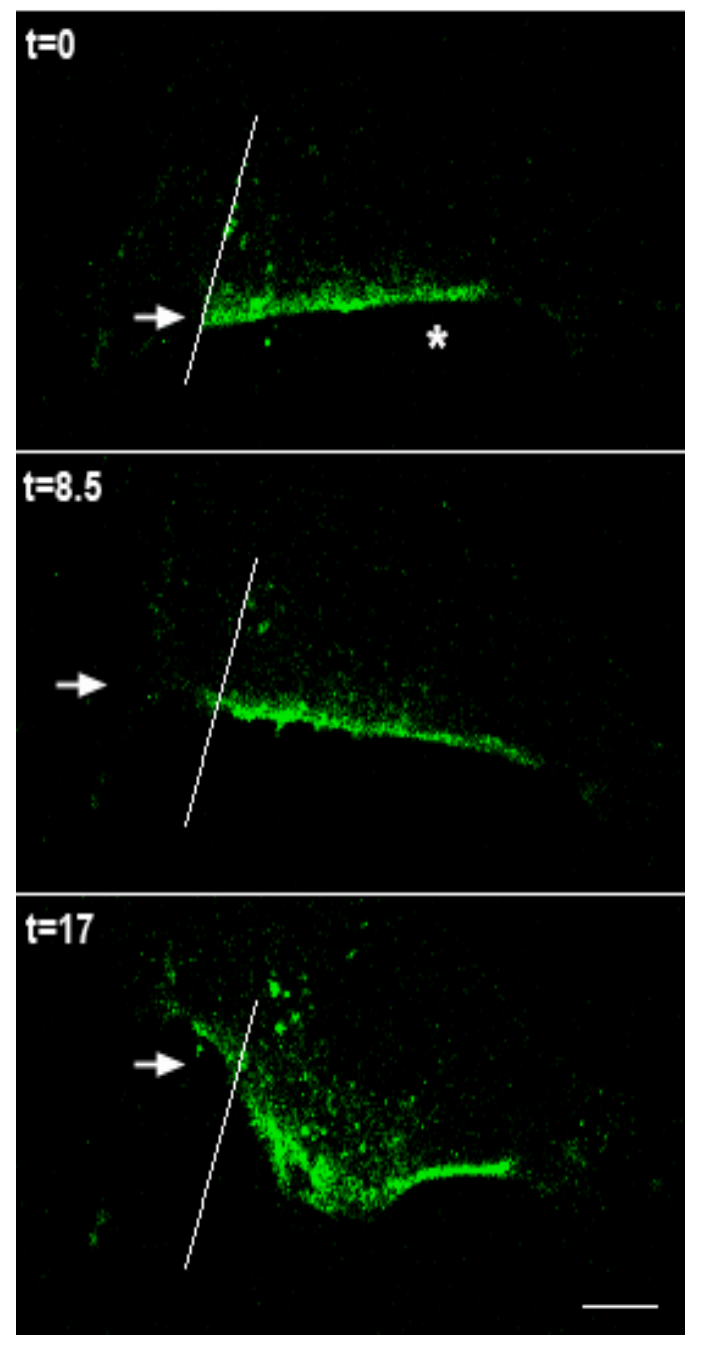

Figure 5 Incorporation of caveolin-1-GFP fluorescence in retracting areas of the trailing edge. (a) Frames taken from a time series demonstrate appearance of caveolin-1-GFP lateral to extant signal. The diagonal mark is a fiduciary line marking the edge of caveolin- 1 polarization at $t=0$. Scale bar, $10 \mu \mathrm{m}$. 


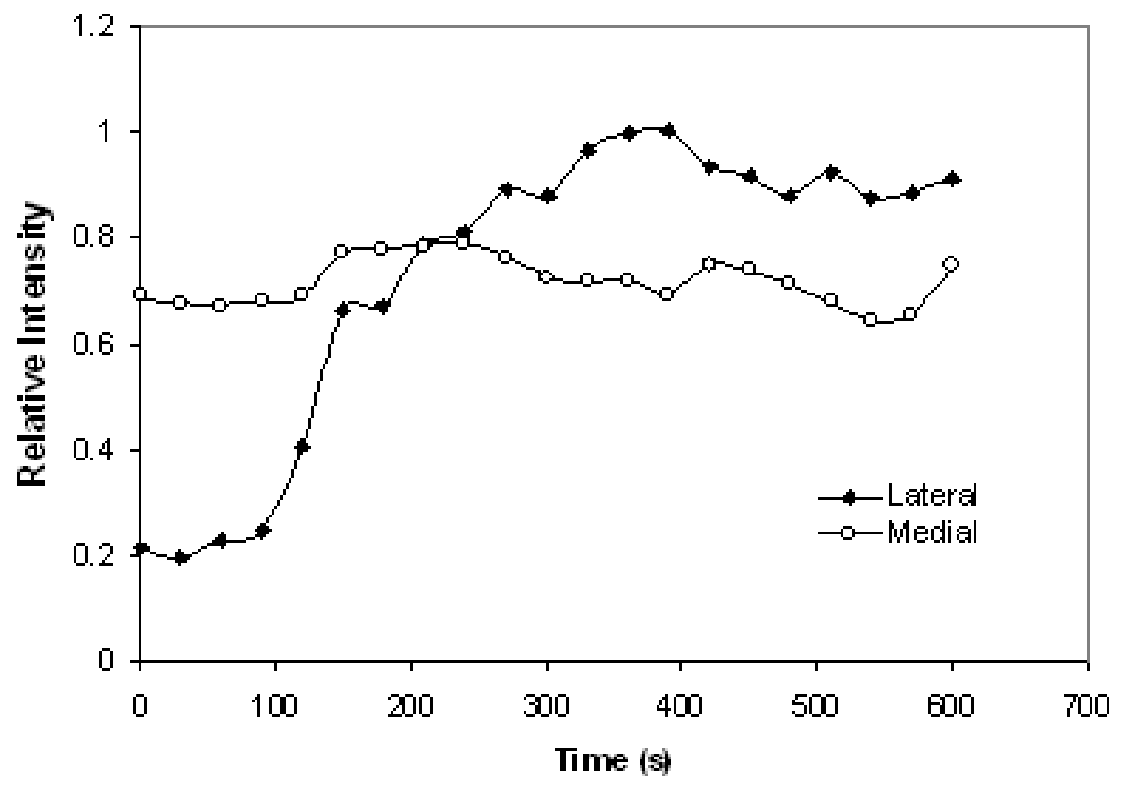

Figure 5 Incorporation of caveolin-1-GFP fluorescence in retracting areas of the trailing edge. (b) Analysis of fluorescent intensity dynamics in the region immediately lateral to polarized caveolin (lateral, see arrows in a). The latter 10 min of the time series in (a) are shown in the graph. Fluorescence intensity of the area was recorded every 30 seconds. A marked increase in intensity is seen, coincident with cell retraction. Fluorescent intensity of a region of extant polarized caveolin-GFP is included for comparison (medial, see asterisk in a). Intensity of this region remains stable throughout the interval. 

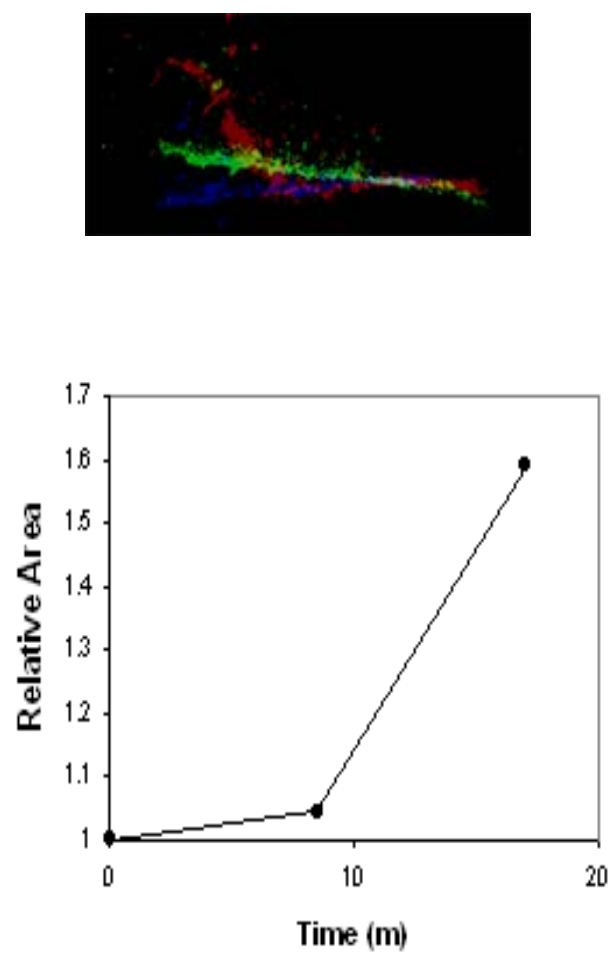

Figure 5 Incorporation of caveolin-1-GFP fluorescence in retracting areas of the trailing edge. (c) A color overlay and (d) a plot of relative areas of polarized caveolin-1 from the frames shown in (a) verify nascent incorporation of caveolin-GFP, rather than migration of the polarized region beyond the fiduciary mark. In the overlay, the initial, intermediate and final time points are represented by blue, green, and red, respectively. 


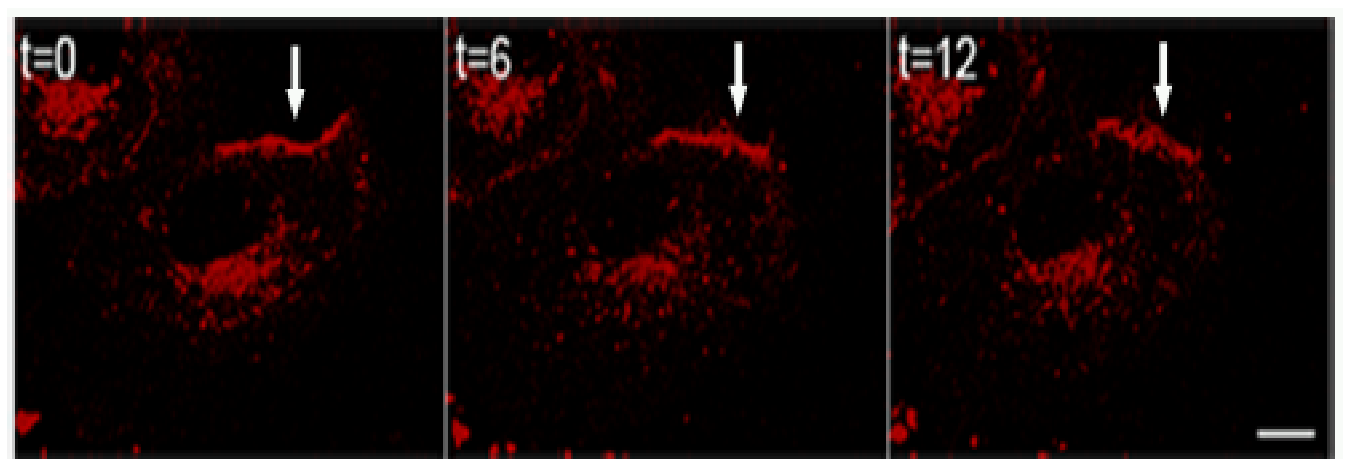

Figure 6 CTB-TRITC labeled GM1 is stable at the cell perimeter. Cells were preincubated with CTB-TRTIC, washed, and allowed to migrate. CTB signal was detected polarized at the cell rear (arrows). This signal remained stable as the cell migrating, indicating that constitutive GM1 endocytosis did not occur. 


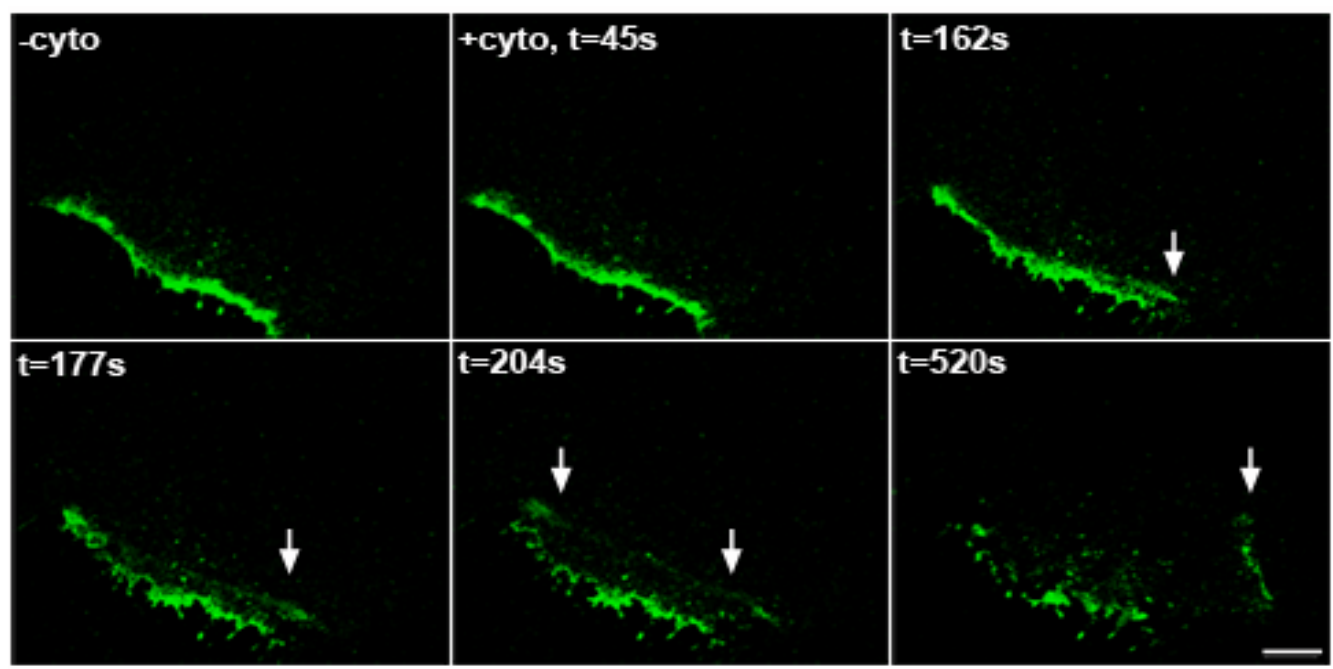

Figure 7 Disruption of caveolin-1 polarization via cytoskeletal disruption. (a) Live cell imaging of polarized caveolin-1-GFP in a motile cell was performed before(-cyto) and after treatment (+cyto) with $50 \mathrm{nM}$ cytochalasin $\mathrm{D}$. Approximately $2.5 \mathrm{~min}$ after treatment, partial detachment of actin-associated caveolin-GFP became evident ( $\mathrm{t}=162)$. Later, an entire rod of caveolin-1-GFP signal detached from the rear, leaving residual caveolin-1-GFP at the rear (arrows, $t=204$ ). After going out of focus, the caveolin-GFP rod appears at the front of the cell, at less than half its original size. Scale bar, $10 \mu \mathrm{m}$. 


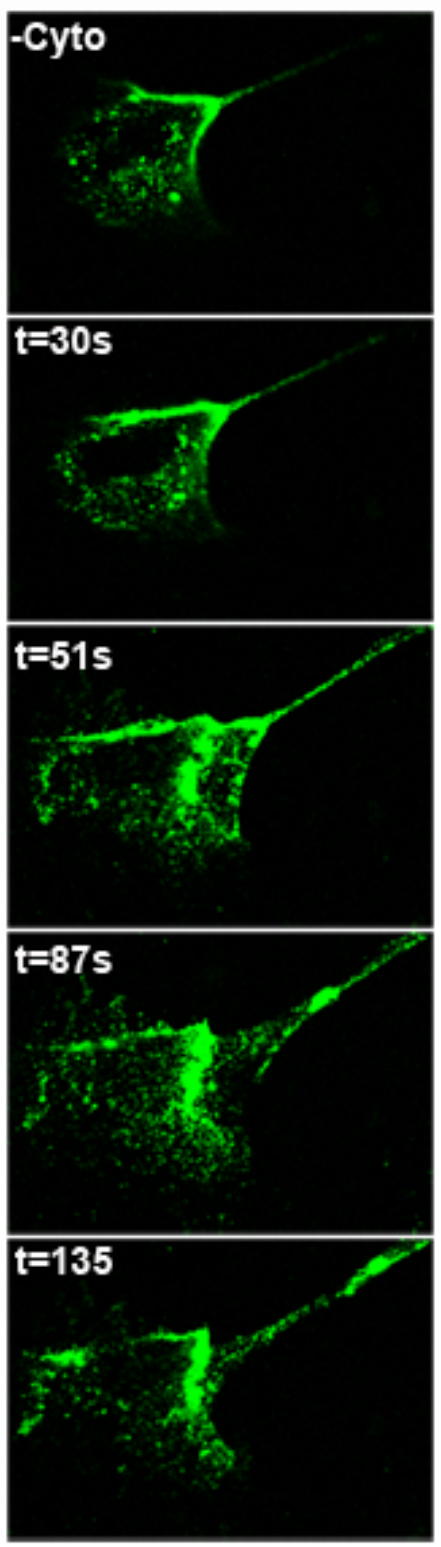

Figure 7 Disruption of caveolin-1 polarization via cytoskeletal disruption. (b) A second example of the effect cytochalasin treatment on polarized caveolin. In this case, caveolin partially rips towards the rear of the cell. Note what appears to be a duplicate signal at $\mathrm{t}=51 \mathrm{~s}$. Some residual caveolin-1 signal remains, but disaggregates into discreet puncta. 
A

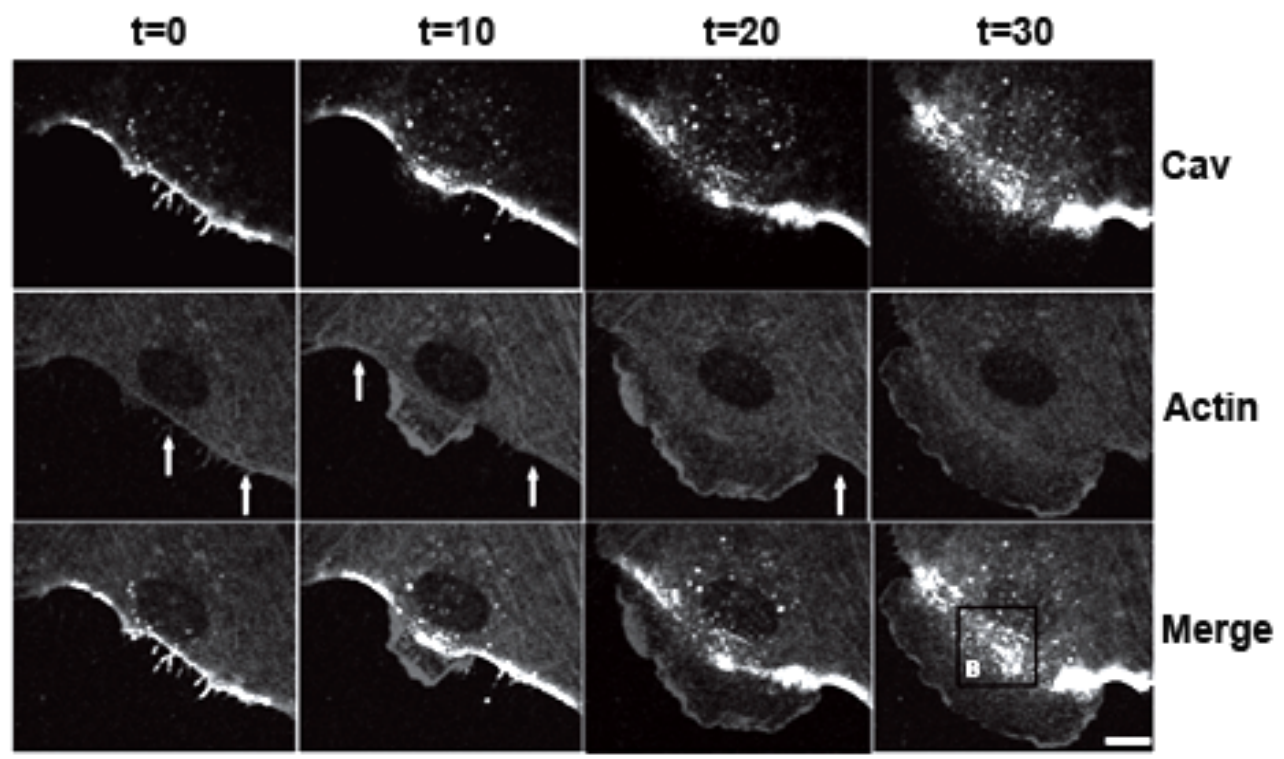

B

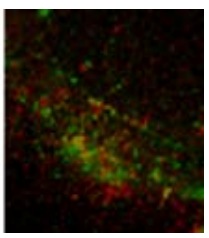

Figure 8 Lamellipodial outgrowth from the cell rear results in caveolin-1 and actin stress fiber dispersion. (A) A time series of a HUVEC coexpressing caveolin-GFP and actin-RFP demonstrates outgrowth of a lamellipodium from the cell rear over a 30 minute period. Initially, caveolin-1 appears co-aligned with actin stress fibers at the rear. At $t=10$ minutes, however, a distinct lamellipodium enriched in cortical actin along the leading edge has begun to bloom from the cell rear. Concomitantly, caveolin-1-GFP signal begins to disperse, and the actin

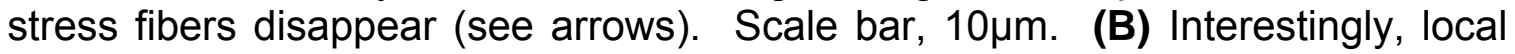
and frenetic movement of caveolin-1-GFP puncta ensues with depolarization, shown here by an overlay of sequential frames. Note that the majority of the puncta do not overlap, revealing their rapid, short-range movement. 


\section{REFERENCES}

Anderson, H. A., Y. Chen, et al. (1996). "Bound simian virus 40 translocates to caveolin-enriched membrane domains, and its entry is inhibited by drugs that selectively disrupt caveolae." Mol. Biol. Cell 7: 1825-1834.

Anderson, R. G. (1998). "The caveolae membrane system." Annu Rev Biochem 67: 199-225.

Anderson, R. G. W. (1993). "Caveolae: Where incoming and outgoing messengers meet." Proc. Natl. Acad. Sci., USA 90: 10909-10913.

Beardsley, A., K. Fang, et al. (2005). "Loss of caveolin-1 polarity impedes endothelial cell polarization and directional movement." J Biol Chem 280(5): 3541-7.

Couet, J., M. Sargiacomo, et al. (1997). "Interaction of a receptor tyrosine kinase, EGF-R, with caveolins. Caveolin binding negatively regulates tyrosine and serine/threonine kinase activities." J. Biol. Chem. 272: 30429-30438.

Dupree, P., R. G. Parton, et al. (1993). "Caveolae and sorting of the trans-Golgi network of epithelial cells." EMBO J. 12: 1597-1605.

Ezratty, E. J., M. A. Partridge, et al. (2005). "Microtubule-induced focal adhesion disassembly is mediated by dynamin and focal adhesion kinase." Nat Cell Biol 7(6): 581-90.

Hecker, M., A. Mulsch, et al. (1994). "Subcellular localization and characterization of nitric oxide synthase(s) in endothelial cells: physiological implications." Biochem. J. 299: 247-252.

Hommelgaard, A. M., K. Roepstorff, et al. (2005). "Caveolae: stable membrane domains with a potential for internalization." Traffic 6(9): 720-4.

Ingber, D. E. (1997). "Tensegrity: the architectural basis of cellular mechanotransduction." Annu Rev Physiol 59: 575-99.

Isshiki, M., J. Ando, et al. (2002). "Sites of $\mathrm{Ca}(2+)$ wave initiation move with caveolae to the trailing edge of migrating cells." J Cell Sci 115(Pt 3): 475-84.

Kaverina, I., O. Krylyshkina, et al. (1999). "Microtubule targeting of substrate contacts promotes their relaxation and dissociation." J Cell Biol 146(5): 1033-44. 
Lisanti, M. P., S. Li, et al. (1996). Caveolin, an integral membrane protein component of caveolae membranes: Implications for signal transduction. Alpha-2 Adrenergic Receptors, Harwood Academic Publishers: pp 63-76.

Lisanti, M. P., P. Scherer, et al. (1994). "Caveolae, caveolin and caveolin-rich membrane domains: A signalling hypothesis." Trends In Cell Biology 4: 231235.

Lisanti, M. P., Z.-L. Tang, et al. (1994). Caveolae: Portals for Transmembrane Signalling and Cellular Transport. Membrane Protein Transport. S. Rothman. Greenwich, CT, JAI Press. In Press.

McIntosh, D. P. and J. E. Schnitzer (1999). "Caveolae require intact VAMP for targeted transport in vascular endothelium." Am J Physiol 277(6 Pt 2): H2222-32.

Mundy, D. I., T. Machleidt, et al. (2002). "Dual control of caveolar membrane traffic by microtubules and the actin cytoskeleton." J Cell Sci 115(Pt 22): 4327-39.

Oh, P. and J. E. Schnitzer (2001). "Segregation of heterotrimeric G proteins in cell surface microdomains. $G(q)$ binds caveolin to concentrate in caveolae, whereas G(i) and G(s) target lipid rafts by default." Mol Biol Cell 12(3): 68598.

Parat, M. O., B. Anand-Apte, et al. (2003). "Differential caveolin-1 polarization in endothelial cells during migration in two and three dimensions." Mol Biol Cell 14(8): 3156-68.

Pelkmans, L., D. Puntener, et al. (2002). "Local actin polymerization and dynamin recruitment in SV40-induced internalization of caveolae." Science 296(5567): 535-9.

Pelkmans, L. and M. Zerial (2005). "Kinase-regulated quantal assemblies and kissand-run recycling of caveolae." Nature 436(7047): 128-33.

Rothberg, K. G., J. E. Heuser, et al. (1992). "Caveolin, a protein component of caveolae membrane coats." Cell 68: 673-682.

Scherer, P. E., T. Okamoto, et al. (1996). "Identification, sequence, and expression of caveolin-2 defines a caveolin gene family." Proc Natl Acad Sci U S A 93(1): 131-5.

Sessa, W. C. (2005). "Regulation of endothelial derived nitric oxide in health and disease." Mem Inst Oswaldo Cruz 100 Suppl 1: 15-8. 
Sonveaux, P., P. Martinive, et al. (2004). "Caveolin-1 expression is critical for vascular endothelial growth factor-induced ischemic hindlimb collateralization and nitric oxide-mediated angiogenesis." Circ Res 95(2): 154-61.

Stahlhut, M. and B. van Deurs (2000). "Identification of filamin as a novel ligand for caveolin-1: evidence for the organization of caveolin-1-associated membrane domains by the actin cytoskeleton." Mol Biol Cell 11(1): 325-37.

Stan, R. V. (2005). "Structure of caveolae." Biochim Biophys Acta 1746(3): 334-48.

Tagawa, A., A. Mezzacasa, et al. (2005). "Assembly and trafficking of caveolar domains in the cell: caveolae as stable, cargo-triggered, vesicular transporters." J Cell Biol 170(5): 769-79.

Thomsen, P., K. Roepstorff, et al. (2002). "Caveolae are highly immobile plasma membrane microdomains, which are not involved in constitutive endocytic trafficking." Mol Biol Cell 13(1): 238-50.

Travis, J. (1993). "Cell biologists explore 'tiny caves'." Science 262: 1208-1209.

Vey, M., S. Pilkuhn, et al. (1996). "Subcellular localization of the scrapie prion proteins in caveolae-like membranous domains." Proc. Natl. Acad. Sci. USA 93: 14945-14949.

Woodman, S. E., A. W. Ashton, et al. (2003). "Caveolin-1 knockout mice show an impaired angiogenic response to exogenous stimuli." Am J Pathol 162(6): 2059-68.

Yu, J., S. Bergaya, et al. (2006). "Direct evidence for the role of caveolin-1 and caveolae in mechanotransduction and remodeling of blood vessels." $\mathrm{J}$ Clin Invest 116(5): 1284-91. 


\section{General Discussion}

We have described a novel role for caveolin and caveolae in cell mechanotransduction. During cell migration, actin stress fibers become distally anchored to trailing edge focal adhesions. Contraction of such fibers induces a strain on such adhesions that normally results in adhesion maturation (Ballestrem, Hinz et al. 2001); however, these adhesions paradoxically disassemble. We have shown that focal adhesion disassembly is predisposed by caveolin-1 recruitment. Moreover, the stable interaction between actin stress fibers and trailing adhesions is replaced by a stable interaction between stress fibers and caveolin-1/caveolae. Thus, we propose that cells generally employ caveolin in the relief of cell-substrate strain at the perimeter. Importantly, our data are consistent with the caveolin-null phenotype, i.e., observed deficits in angiogenesis and vascular remodeling. Woodman et al. reported defects in the ability of caveolin-1 null endothelial cells to infiltrate growth-factor supplemented Matrigel plugs implanted into knockout mice (Woodman, Ashton et al. 2003). Using an adaptive angiogenic model, Sonveaux et al. observed serious angiogenic defects, resulting in ischemia and tissue death in caveolin-1 null animals (Sonveaux, Martinive et al. 2004). Lastly, genetic ablation of caveolin-1 results in defective vascular remodeling in response to changes in shear rate in vivo (Yu, Bergaya et al. 2006). These phenotypes are neither predicted nor adequately explained by current dogma regarding caveolin function.

The overall goal of this project was to address the function of caveolin-1 in migrating cells. Early on, immunofluorescent staining revealed striking 
polarization of endogenous caveolin-1 to the rear of migrating endothelial cells. Analysis of caveolin polarization in the context of the cytoskeleton in conjunction with live cell imaging has provided important clues in the function of caveolin during migration.

Study 1:

Randomly-moving and chemotactic HUVECs exhibit caveolin-1 polarization to the cell rear. Areas of caveolin concentration at the cell perimeter are notably devoid of focal adhesion signal and lamellipodial protrusion. In contrast, HUVECs allowed to spread for a short time appear circular, with caveolin-1 signal concentrated in the center of the cell, and focal adhesions decorate the circumference of the circular lamellipodium. Thus, a transition between circular to polarized cell morphology must exist; polarization of caveolin must also exist, leading us to hypothesize that caveolin polarization may actively contribute to cell polarity. To test this hypothesis, we transfected HUVECs with small, interfering RNA against caveolin-1, which specifically and dramatically reduced caveolin-1 protein expression. We observed a deficit in cell polarization with caveolin reduction, which is consistent with our hypothesis. Moreover, we observed an deficit in cell migration with caveolin knockdown, suggesting a positive role for caveolin-1 in cell motility.

Study 2:

To visualize the circular-to-polarized morphology transition simultaneously with caveolin-1 translocation, we used combined DIC and fluorescent live cell imaging in HUVECs expressing chimeric caveolin-1-GFP. While we observed 
mutual exclusion of caveolin and focal adhesion staining in the previous study, we noticed a brief and catastrophic recruitment of caveolin-1 to focal adhesions coincident with cell polarization in live cells. We verified this chaotropic interaction of caveolin-1 with focal adhesions in cells co-expressing fluorescenttagged caveolin-1 and paxillin. We found that recruitment of caveolin within $300 \mathrm{~nm}$ of an adhesion predisposed its disassembly. Conversely, adhesions remained stable beyond $300 \mathrm{~nm}$ of caveolin- 1 signal. Moreover, we showed that caveolin associates with the distal tips of actin stress fibers prior to their recoil and collapse along with the trailing edge. Consistent with these results, we demonstrated a defect in tail detachment in motile, caveolin-1 null MEFs. Contractility in these cells was not significantly reduced, suggesting that tail persistence is specifically a result of defective detachment with caveolin ablation. This defect resulted in a decrease in overall chemokinetic motility with caveolin-1 ablation; however, cell ripping, which obviously short circuits the need for adhesion disassembly was observed frequently in MEFs, obviously confounding the effects of caveolin-1 knockout. Interestingly, caveolin ablation resulted in a slight but significant increase in directional persistence of MEFs, consistent with a report that anchorage of the cell rear was necessary for continuous unidirectional movement of fibroblasts. Increased persistence despite decreased overall movement suggests that caveolin-1-mediated adhesion disassembly functions in the plasticity of movement in MEFs. 
Study 3:

In Study 2, we characterized caveolin as it was polarizing and being recruited to the trailing edge. In Study 3 , we characterized caveolin and its association with actin after polarization has occurred. We observed that perimeter-association of caveolin-1 and caveolae was specifically induced by cell motility. Live cell imaging of HUVECs co-expressing caveolin-1 and actin revealed that caveolin stably associates with stress fibers over relatively long time periods. We showed by inhibition of non-muscle myosin by blebbistatin that caveolin-1 polarization is initially contingent on cell contractility. Photobleaching of polarized caveolin-1-GFP reveals little or no lateral movement of signal from neighboring areas, resulting in negligible FRAP, and suggesting tethering of caveolae to actin at the cell rear. Consistent with this, CTB-TRITC-labeled GM1 was stable at the rear of migrating cells with the caveat of unidirectional movement. Lastly, pharmacologic or cell-induced actin stress fiber disassembly at the cell rear resulted in depolarization of caveolin signal, which disaggregated into frenzied puncta.

Taken together, our data indicate that caveolin-1 disassembles overstrained focal adhesions at the cell perimeter. At the present, the mechanism of caveolin-1 recruitment and affect on adhesion structure remain unknown. An attractive possibility is transit of caveolin-1 or caveolae on microtubules that terminate at strained focal adhesions. Kaverina et al. have reported that microtubules polymerize toward areas of cell strain and specifically target focal adhesions (Kaverina, Krylyshkina et al. 1999). Moreover, Tagawa et al. have 
observed rapid, long range movement of caveolin-1 GFP speckles consistent with microtubule trafficking (Tagawa, Mezzacasa et al. 2005). Our own attempts to exogenously express tubulin-GFP and visualize the microtubule cytoskeleton have not successfully demonstrated a temporal and spatial recruitment to the trailing edge. Instead, the microtubules seem to associate constitutively at the cell perimeter. This result could be an artifact of overexpression, although exogenous expression of all our constructs by lipid-based transfection was estimated to be less than $5 \%$ of endogenous levels. Disruption of the microtubule cytoskeleton is not a valid method to determine if caveolin-1 is trafficked on microtubules; such disruption globally affects cell tensegrity and motility (Ballestrem, Wehrle-Haller et al. 2000).

Another problem is the poor fluorescence of non-aggregated caveolinGFP. In our observations, caveolin-1 signal was originally observed as it coalesced at the retracting trailing edge. Thus, its transit from the cell interior was not appreciated. This raises an interesting point-a further level of organization of caveolin-1/caveolae may exist at the trailing edge. Caveolin-GFP fluorescence appears very intense upon polarization. Upon depolarization by actin fiber depolymerization, caveolin-1-GFP signal appears as disaggregated puncta. Had these puncta formed prior to polarization, we should have seen at least a fraction of them en route to the retracting trailing edge. The presence of such puncta after, but not before caveolin-1 polarization, suggests another level of organization of caveolae at the cell perimeter, beyond what occurs in the ER and Golgi apparatus (see Literature Review). 
Electron microscopy studies have identified caveolae in grape-like clusters at the cell membrane (Stan 2005). The puncta observed in our studies may represent such clusterings of caveolae. This raises an interesting mechanistic possibility. We have demonstrated that a stable actin-adhesion interaction is supplanted by stable interaction of the same actin with caveolin. Thus, caveolin essentially competes with adhesions for actin association. Assembly of such caveolae clusters may simultaneously bind up all available actin fibers, overwhelming the actin-adhesion interaction. The speculation that the caveolar "coat" is actually composed of actin (Stan 2005) seems to support this idea.

The discovery of caveolin as a "mechano-effector" that rearranges cell morphology in response to excess strain may have interesting ramifications in physiology and pathology. As mentioned previously, caveolin knockout mice experience pulmonary hypertension and hypercellularity as they develop. Razani et al. point out that this vascular hypercellularity is analogously encountered in fetal lung parenchyma as a reaction to increased mechanical stress and strain intrinsic to the breathing process (Liu, Tanswell et al. 1999), and suggest that lung caveolae may function in mitigating such mechanical forces in adulthood. Lack of caveolin-mediated adhesion disassembly to modify cell-substrate rearrangements strained by development sufficiently explains this phenotype.

It is interesting to speculate whether caveolin-3 functions analogously to caveolin-1 in costameric rearrangement in muscle cells. Dominant-negative mutations in caveolin-3 are well associated with certain muscular dystrophies, although the function of caveolin-3 in striated muscle is not conclusively known 
(Woodman, Sotgia et al. 2004). If muscle cells cannot adapt to developmental strains, (i.e. by disassembly of strained costameres) it is plausible that cell membrane damage and apoptosis might result. Thus our study may indirectly shed light on the pathogenesis of certain muscular dystrophies.

In conclusion, strain can develop at perimeter-associated focal adhesions which can be sensed by the cell and modified through the recruitment of caveolin-1. We propose that caveolin-1 functions to maintain appropriate cellular tensegrity during development and cell migration by contributing to focal adhesion disassembly. Thus caveolin contributes to the integrity of tissues by preventing the generation of inordinate strain at the cellular level. 


\section{General References}

Anderson, H. A., Y. Chen, et al. (1996). "Bound simian virus 40 translocates to caveolinenriched membrane domains, and its entry is inhibited by drugs that selectively disrupt caveolae." Mol. Biol. Cell 7: 1825-1834.

Anderson, R. G. (1998). "The caveolae membrane system." Annu Rev Biochem 67: 199225.

Anderson, R. G., B. A. Kamen, et al. (1992). "Potocytosis: sequestration and transport of small molecules by caveolae." Science 255(5043): 410-1.

Anderson, R. G. W. (1993). "Caveolae: Where incoming and outgoing messengers meet." Proc. Natl. Acad. Sci., USA 90: 10909-10913.

Anderson, R. G. W. (1998). "The caveolae membrane system." Ann. Rev. Biochem. 67: 199-225.

Aplin, A. E., A. Howe, et al. (1998). "Signal transduction and signal modulation by cell adhesion receptors: the role of integrins, cadherins, immunoglobulin-cell adhesion molecules, and selectins." Pharmacol Rev 50(2): 197-263.

Aplin, A. E., A. K. Howe, et al. (1999). "Cell adhesion molecules, signal transduction and cell growth." Curr Opin Cell Biol 11(6): 737-44.

Ashton, A. W., R. Yokota, et al. (1999). "Inhibition of endothelial cell migration, intercellular communication, and vascular tube formation by thromboxane A(2)." J Biol Chem 274(50): 35562-70.

Avraham, S., R. London, et al. (1995). "Identification and characterization of a novel related adhesion focal tyrosine kinase (RAFTK) from megakaryocytes and brain." J Biol Chem 270(46): 27742-51.

Ballestrem, C., B. Hinz, et al. (2001). "Marching at the front and dragging behind: differential alphaVbeta3-integrin turnover regulates focal adhesion behavior." $\underline{\mathrm{J}}$ Cell Biol 155(7): 1319-32.

Ballestrem, C., B. Wehrle-Haller, et al. (2000). "Actin-dependent lamellipodia formation and microtubule-dependent tail retraction control-directed cell migration." $\underline{\mathrm{Mol}}$ Biol Cell 11(9): 2999-3012.

Beardsley, A., K. Fang, et al. (2005). "Loss of caveolin-1 polarity impedes endothelial cell polarization and directional movement." J Biol Chem 280(5): 3541-7.

Beningo, K. A., M. Dembo, et al. (2001). "Nascent focal adhesions are responsible for the generation of strong propulsive forces in migrating fibroblasts." $\underline{\mathrm{J} \text { Cell Biol }}$ 153(4): 881-8. 
Birge, R. B., J. E. Fajardo, et al. (1993). "Identification and characterization of a highaffinity interaction between v-Crk and tyrosine-phosphorylated paxillin in CT10transformed fibroblasts." Mol Cell Biol 13(8): 4648-56.

Brakebusch, C. and R. Fassler (2003). "The integrin-actin connection, an eternal love affair." Embo J 22(10): 2324-33.

Braren, R., H. Hu, et al. (2006). "Endothelial FAK is essential for vascular network stability, cell survival, and lamellipodial formation." J Cell Biol 172(1): 151-62.

Brown, N. H., S. L. Gregory, et al. (2002). "Talin is essential for integrin function in Drosophila." Dev Cell 3(4): 569-79.

Bruns, R. R. and G. E. Palade (1968). "Studies on blood capillaries. I. General organization of blood capillaries in muscle." J Cell Biol 37(2): 244-76.

Bruns, R. R. and G. E. Palade (1968). "Studies on blood capillaries. II. Transport of ferritin molecules across the wall of muscle capillaries." J Cell Biol 37(2): 277-99.

Bucci, M., J. P. Gratton, et al. (2000). "In vivo delivery of the caveolin-1 scaffolding domain inhibits nitric oxide synthesis and reduces inflammation." Nat Med 6(12): 1362-7.

Burridge, K. and M. Chrzanowska-Wodnicka (1996). "Focal adhesions, contractility, and signaling." Annu Rev Cell Dev Biol 12: 463-518.

Cao, G., G. Yang, et al. (2003). "Disruption of the caveolin-1 gene impairs renal calcium reabsorption and leads to hypercalciuria and urolithiasis." Am J Pathol 162(4): 1241-8.

Cao, H., A. R. Sanguinetti, et al. (2004). "Oxidative stress activates both Src-kinases and their negative regulator Csk and induces phosphorylation of two targeting proteins for Csk: caveolin-1 and paxillin." Exp Cell Res 294(1): 159-71.

Carragher, N. O., V. J. Fincham, et al. (2001). "Cleavage of focal adhesion kinase by different proteases during SRC-regulated transformation and apoptosis. Distinct roles for calpain and caspases." J Biol Chem 276(6): 4270-5.

Chen, H., D. M. Cohen, et al. (2005). "Spatial distribution and functional significance of activated vinculin in living cells." J Cell Biol 169(3): 459-70.

Chen, H. C. and J. L. Guan (1994). "Stimulation of phosphatidylinositol 3'-kinase association with foca adhesion kinase by platelet-derived growth factor." J Biol Chem 269(49): 31229-33.

Chen, W. T. (1981). "Mechanism of retraction of the trailing edge during fibroblast movement." J Cell Biol 90(1): 187-200. 
Chrzanowska-Wodnicka, M. and K. Burridge (1996). "Rho-stimulated contractility drives the formation of stress fibers and focal adhesions." J Cell Biol 133(6): 1403-15.

Clevenger, C. V. and J. B. Kline (2001). "Prolactin receptor signal transduction." Lupus 10(10): 706-18.

Cohen, A. W., T. P. Combs, et al. (2003). "Role of caveolin and caveolae in insulin signaling and diabetes." Am J Physiol Endocrinol Metab 285(6): E1151-60.

Cohen, A. W., R. Hnasko, et al. (2004). "Role of caveolae and caveolins in health and disease." Physiol Rev 84(4): 1341-79.

Cohen, A. W., B. Razani, et al. (2003). "Caveolin-1-deficient mice show insulin resistance and defective insulin receptor protein expression in adipose tissue." Am J Physiol Cell Physiol 285(1): C222-35.

Conrad, P. A., E. J. Smart, et al. (1995). "Caveolin cycles between plasma membrane caveolae and the Golgi complex by microtubule-dependent and microtubuleindependent steps." J Cell Biol 131(6 Pt 1): 1421-33.

Couet, J., M. Sargiacomo, et al. (1997). "Interaction of a receptor tyrosine kinase, EGF-R, with caveolins. Caveolin binding negatively regulates tyrosine and serine/threonine kinase activities." J. Biol. Chem. 272: 30429-30438.

Craig, S. W. and J. V. Pardo (1983). "Gamma actin, spectrin, and intermediate filament proteins colocalize with vinculin at costameres, myofibril-to-sarcolemma attachment sites." Cell Motil 3(5-6): 449-62.

Damm, E. M., L. Pelkmans, et al. (2005). "Clathrin- and caveolin-1-independent endocytosis: entry of simian virus 40 into cells devoid of caveolae." $\underline{\mathrm{J} \text { Cell Biol }}$ 168(3): 477-88.

Das, K., R. Y. Lewis, et al. (1999). "The membrane spanning domains of caveolins 1 and 2 mediate the formation of caveolin hetero-oligomers. Implications for the assembly of caveolae membranes in vivo." J. Biol. Chem. 274: 18721-18726.

Das, K., R. Y. Lewis, et al. (1999). "The membrane-spanning domains of caveolins-1 and -2 mediate the formation of caveolin hetero-oligomers. Implications for the assembly of caveolae membranes in vivo." J Biol Chem 274(26): 18721-8.

del Pozo, M. A., N. Balasubramanian, et al. (2005). "Phospho-caveolin-1 mediates integrin-regulated membrane domain internalization." Nat Cell Biol 7(9): 901-8.

Drab, M., P. Verkade, et al. (2001). "Loss of caveolae, vascular dysfunction, and pulmonary defects in caveolin-1 gene-disrupted mice." Science 293(5539): 244952. 
Dunphy, J. T. and M. E. Linder (1998). "Signalling functions of protein palmitoylation." Biochim Biophys Acta 1436(1-2): 245-61.

Dupree, P., R. G. Parton, et al. (1993). "Caveolae and sorting of the trans-Golgi network of epithelial cells." EMBO J. 12: 1597-1605.

Engelman, J. A., X. L. Zhang, et al. (1998). "Chromosomal localization, genomic organization, and developmental expression of the murine caveolin gene family (Cav-1, -2, and -3). Cav-1 and Cav-2 genes map to a known tumor suppressor locus (6-A2/7q31)." FEBS Lett 429(3): 330-6.

Engelman, J. A., X. L. Zhang, et al. (1998). "Genes encoding human caveolin-1 and -2 are co-localized to the D7S522 locus (7q31.1), a known fragile site (FRA7G) that is frequently deleted in human cancers." FEBS Lett 436(3): 403-10.

Ezratty, E. J., M. A. Partridge, et al. (2005). "Microtubule-induced focal adhesion disassembly is mediated by dynamin and focal adhesion kinase." Nat Cell Biol 7(6): 581-90.

Feron, O., L. Belhassen, et al. (1996). "Endothelial nitric oxide synthase targeting to caveolae. Specific interactions with caveolin isoforms in cardiac myocytes and endothelial cells." J Biol Chem 271(37): 22810-4.

Folkman, J. (2002). "Role of angiogenesis in tumor growth and metastasis." Semin Oncol 29(6 Suppl 16): 15-8.

Fra, A. M., E. Williamson, et al. (1995). "De novo formation of caveolae in lymphocytes by expression of VIP21-caveolin." Proc Natl Acad Sci U S A 92(19): 8655-9.

Franco, S. J., M. A. Rodgers, et al. (2004). "Calpain-mediated proteolysis of talin regulates adhesion dynamics." Nat Cell Biol 6(10): 977-83.

Franz, C. M. and D. J. Muller (2005). "Analyzing focal adhesion structure by atomic force microscopy." J Cell Sci 118(Pt 22): 5315-23.

Fujimoto, T., A. Miyawaki, et al. (1995). "Inositol 1,4,5-trisphosphate receptor-like protein in plasmalemmal caveolae is linked to actin filaments." J Cell Sci $\mathbf{1 0 8 ~ ( ~ P t ~}$ 1): $7-15$.

Fukami, K., T. Endo, et al. (1994). "alpha-Actinin and vinculin are PIP2-binding proteins involved in signaling by tyrosine kinase." J Biol Chem 269(2): 1518-22.

Fulton, D., J. P. Gratton, et al. (2001). "Post-translational control of endothelial nitric oxide synthase: why isn't calcium/calmodulin enough?" J Pharmacol Exp Ther 299(3): 818-24.

Gabella, G. (1978). "Inpocketings of the cell membrane (caveolae) in the rat myocardium." J Ultrastruct Res 65(2): 135-47. 
Galbiati, F., J. A. Engelman, et al. (2001). "Caveolin-3 null mice show a loss of caveolae, changes in the microdomain distribution of the dystrophin-glycoprotein complex, and t-tubule abnormalities." J Biol Chem 276(24): 21425-33.

Galbraith, C. G. and M. P. Sheetz (1997). "A micromachined device provides a new bend on fibroblast traction forces." Proc Natl Acad Sci U S A 94(17): 9114-8.

Galbraith, C. G. and M. P. Sheetz (1998). "Forces on adhesive contacts affect cell function." Curr Opin Cell Biol 10(5): 566-71.

Galvez, B. G., S. Matias-Roman, et al. (2004). "Caveolae are a novel pathway for membrane-type 1 matrix metalloproteinase traffic in human endothelial cells." Mol Biol Cell 15(2): 678-87.

Garcia-Alvarez, B., J. M. de Pereda, et al. (2003). "Structural determinants of integrin recognition by talin." Mol Cell 11(1): 49-58.

Garcia-Cardena, G., P. Oh, et al. (1996). "Targeting of nitric oxide synthase to endothelial cell caveolae via palmitoylation: implications for nitric oxide signaling." Proc Natl Acad Sci U S A 93(13): 6448-53.

Gingras, D., F. Gauthier, et al. (1998). "Localization of RhoA GTPase to endothelial caveolae-enriched membrane domains." Biochem Biophys Res Commun 247(3): 888-93.

Glenney, J. R., Jr. (1989). "Tyrosine phosphorylation of a 22-kDa protein is correlated with transformation by Rous sarcoma virus." J Biol Chem 264(34): 20163-6.

Glenney, J. R., Jr. and L. Zokas (1989). "Novel tyrosine kinase substrates from Rous sarcoma virus-transformed cells are present in the membrane skeleton." $\underline{\mathrm{J} \text { Cell }}$ Biol 108(6): 2401-8.

Glenney, J. R. and L. Zokas (1989). "Novel tyrosine kinase substrates from Rous sarcoma virus transformed cells are present in the membrane cytoskeleton." J. Cell Biol. 108: 2401-2408.

Gorodinsky, A. and D. A. Harris (1995). "Glycolipid-anchored proteins in neuroblastoma cells form detergent-resistant complexes without caveolin." J Cell Biol 129(3): $619-27$.

Griffoni, C., E. Spisni, et al. (2000). "Knockdown of caveolin-1 by antisense oligonucleotides impairs angiogenesis in vitro and in vivo." Biochem. Biophys. Res. Commun. 276: 756-761.

Hagiwara, Y., T. Sasaoka, et al. (2000). "Caveolin-3 deficiency causes muscle degeneration in mice." Hum Mol Genet 9(20): 3047-54. 
Halayko, A. J. and G. L. Stelmack (2005). "The association of caveolae, actin, and the dystrophin-glycoprotein complex: a role in smooth muscle phenotype and function?" Can J Physiol Pharmacol 83(10): 877-91.

Han, D. C. and J. L. Guan (1999). "Association of focal adhesion kinase with Grb7 and its role in cell migration." J Biol Chem 274(34): 24425-30.

Hasegawa, H., E. Kiyokawa, et al. (1996). "DOCK180, a major CRK-binding protein, alters cell morphology upon translocation to the cell membrane." Mol Cell Biol 16(4): 1770-6.

Hecker, M., A. Mulsch, et al. (1994). "Subcellular localization and characterization of nitric oxide synthase(s) in endothelial cells: physiological implications." Biochem. J. 299: 247-252.

Hemmings, L., D. J. Rees, et al. (1996). "Talin contains three actin-binding sites each of which is adjacent to a vinculin-binding site." J Cell Sci 109 ( Pt 11): 2715-26.

Hildebrand, J. D., M. D. Schaller, et al. (1995). "Paxillin, a tyrosine phosphorylated focal adhesion-associated protein binds to the carboxyl terminal domain of focal adhesion kinase." Mol Biol Cell 6(6): 637-47.

Hnasko, R. and M. P. Lisanti (2003). "The biology of caveolae: lessons from caveolin knockout mice and implications for human disease." Mol Interv 3(8): 445-64.

Hommelgaard, A. M., K. Roepstorff, et al. (2005). "Caveolae: stable membrane domains with a potential for internalization." Traffic 6(9): 720-4.

Horwitz, A. R. and J. T. Parsons (1999). "Cell migration--movin' on." Science 286(5442): 1102-3.

Huang, C., Z. Rajfur, et al. (2003). "JNK phosphorylates paxillin and regulates cell migration." Nature 424(6945): 219-23.

Hynes, R. O. (1992). "Integrins: versatility, modulation, and signaling in cell adhesion." Cell 69(1): 11-25.

Ikezu, T., H. Ueda, et al. (1998). "Affinity-purification and characterization of caveolins from the brain: differential expression of caveolin-1, -2 , and -3 in brain endothelial and astroglial cell types." Brain Res 804(2): 177-92.

Ingber, D. E. (1997). "Integrins, tensegrity, and mechanotransduction." Gravit Space Biol Bull 10(2): 49-55.

Ingber, D. E. (1997). "Tensegrity: the architectural basis of cellular mechanotransduction." Annu Rev Physiol 59: 575-99. 
Isshiki, M., J. Ando, et al. (2002). "Sites of $\mathrm{Ca}(2+)$ wave initiation move with caveolae to the trailing edge of migrating cells." J Cell Sci 115(Pt 3): 475-84.

Izumi, T., Y. Shibata, et al. (1988). "Striped structures on the cytoplasmic surface membranes of the endothelial vesicles of the rat aorta revealed by quick-freeze, deep-etching replicas." Anat Rec 220(3): 225-32.

Izumi, T., Y. Shibata, et al. (1989). "The cytoplasmic surface structures of uncoated vesicles in various tissues of rat as revealed by quick-freeze, deep-etching replicas." J Electron Microsc (Tokyo) 38(1): 47-53.

Izumi, T., Y. Shibata, et al. (1991). "Quick-freeze, deep-etch studies of endothelial components, with special reference to cytoskeletons and vesicle structures." $\underline{\mathrm{J}}$ Electron Microsc Tech 19(3): 316-26.

John, T. A., S. M. Vogel, et al. (2001). "Evidence for the role of alveolar epithelial gp60 in active transalveolar albumin transport in the rat lung." J Physiol 533(Pt 2): 54759.

Johnson, R. P. and S. W. Craig (1994). "An intramolecular association between the head and tail domains of vinculin modulates talin binding." J Biol Chem 269(17): 12611-9.

Ju, H., R. Zou, et al. (1997). "Direct interaction of endothelial nitric-oxide synthase and caveolin-1 inhibits synthase activity." J Biol Chem 272(30): 18522-5.

Kanner, S. B., A. B. Reynolds, et al. (1990). "Monoclonal antibodies to individual tyrosine-phosphorylated protein substrates of oncogene-encoded tyrosine kinases." Proc Natl Acad Sci U S A 87(9): 3328-32.

Kasus-Jacobi, A., V. Bereziat, et al. (2000). "Evidence for an interaction between the insulin receptor and Grb7. A role for two of its binding domains, PIR and SH2." Oncogene 19(16): 2052-9.

Kaverina, I., O. Krylyshkina, et al. (1999). "Microtubule targeting of substrate contacts promotes their relaxation and dissociation." J Cell Biol 146(5): 1033-44.

Kawamura, S., S. Miyamoto, et al. (2003). "Initiation and transduction of stretch-induced RhoA and Rac1 activation through caveolae: cytoskeletal regulation of ERK translocation." J Biol Chem 278(33): 31111-7.

Kirkham, M., A. Fujita, et al. (2005). "Ultrastructural identification of uncoated caveolinindependent early endocytic vehicles." J Cell Biol 168(3): 465-76.

Knudsen, B. S., S. M. Feller, et al. (1994). "Four proline-rich sequences of the guaninenucleotide exchange factor C3G bind with unique specificity to the first Src homology 3 domain of Crk." J Biol Chem 269(52): 32781-7. 
Kroemker, M., A. H. Rudiger, et al. (1994). "Intramolecular interactions in vinculin control alpha-actinin binding to the vinculin head." FEBS Lett 355(3): 259-62.

Kuhn, K. and J. Eble (1994). "The structural bases of integrin-ligand interactions." Trends Cell Biol 4(7): 256-61.

Kurzchalia, T. V., P. Dupree, et al. (1992). "VIP21, a 21-kD membrane protein is an integral component of trans-Golgi-network-derived transport vesicles." $\underline{\mathrm{J} \text { Cell Biol }}$ 118(5): 1003-14.

Labrecque, L., I. Royal, et al. (2003). "Regulation of vascular endothelial growth factor receptor-2 activity by caveolin-1 and plasma membrane cholesterol." Mol Biol Cell 14(1): 334-47.

Le Lay, S. and T. V. Kurzchalia (2005). "Getting rid of caveolins: phenotypes of caveolin-deficient animals." Biochim Biophys Acta 1746(3): 322-33.

Le, P. U., G. Guay, et al. (2002). "Caveolin-1 is a negative regulator of caveolaemediated endocytosis to the endoplasmic reticulum." J Biol Chem 277(5): 3371-9.

Lee, H., D. Volonte, et al. (2000). "Constitutive and growth factor-regulated phosphorylation of caveolin-1 occurs at the same site (Tyr-14) in vivo: identification of a c-Src/Cav-1/Grb7 signaling cassette." Mol Endocrinol 14(11): 1750-75.

Lee, J. and K. Jacobson (1997). "The composition and dynamics of cell-substratum adhesions in locomoting fish keratocytes." J Cell Sci 110 ( Pt 22): 2833-44.

Li, S., R. Seitz, et al. (1996). "Phosphorylation of caveolin by Src tyrosine kinases: The aisoform of caveolin is selectively phosphorylated by v-Src in vivo." J. Biol. Chem. 271: 3863-3868.

Lisanti, M. P., S. Li, et al. (1996). Caveolin, an integral membrane protein component of caveolae membranes: Implications for signal transduction. Alpha-2 Adrenergic Receptors, Harwood Academic Publishers: pp 63-76.

Lisanti, M. P., P. Scherer, et al. (1994). "Caveolae, caveolin and caveolin-rich membrane domains: A signalling hypothesis." Trends In Cell Biology 4: 231-235.

Lisanti, M. P., P. E. Scherer, et al. (1994). "Characterization of caveolin-rich membrane domains isolated from an endothelial-rich source: Implications for human disease." J. Cell Biol. 126: 111-126.

Lisanti, M. P., Z.-L. Tang, et al. (1994). Caveolae: Portals for Transmembrane Signalling and Cellular Transport. Membrane Protein Transport. S. Rothman. Greenwich, CT, JAI Press. In Press. 
Liu, J., P. Oh, et al. (1997). "Organized endothelial cell signal transduction in caveolae." J. Biol. Chem. 272: 7211-7222.

Liu, J., P. Oh, et al. (1997). "Organized endothelial cell surface signal transduction in caveolae distinct from glycosylphosphatidylinositol-anchored protein microdomains." J Biol Chem 272(11): 7211-22.

Liu, J., X. B. Wang, et al. (2002). "Caveolin-1 expression enhances endothelial capillary tubule formation." J Biol Chem. 277: 10661-10668.

Liu, M., A. K. Tanswell, et al. (1999). "Mechanical force-induced signal transduction in lung cells." Am J Physiol 277(4 Pt 1): L667-83.

Liu, P., Y. Ying, et al. (1997). "Platelet-derived growth factor activates mitogen-activated protein kinase in isolated caveolae." Proc Natl Acad Sci U S A 94(25): 13666-70.

Loftus, J. C. and R. C. Liddington (1997). "New insights into integrin-ligand interaction." J Clin Invest 100(11 Suppl): S77-81.

Manes, S., E. Mira, et al. (1999). "Membrane raft microdomains mediate front-rear polarity in migrating cells." Embo J 18(22): 6211-20.

Manes, S., E. Mira, et al. (2000). "Cells on the move: a dialogue between polarization and motility." IUBMB Life 49(2): 89-96.

Mangeat, P. and K. Burridge (1984). "Actin-membrane interaction in fibroblasts: what proteins are involved in this association?" J Cell Biol 99(1 Pt 2): 95s-103s.

Mayer, B. J., M. Hamaguchi, et al. (1988). "Characterization of p47gag-crk, a novel oncogene product with sequence similarity to a putative modulatory domain of protein-tyrosine kinases and phospholipase C." Cold Spring Harb Symp Quant Biol 53 Pt 2: 907-14.

Mayer, C., K. Maaser, et al. (2004). "Release of cell fragments by invading melanoma cells." Eur J Cell Biol 83(11-12): 709-15.

McIntosh, D. P. and J. E. Schnitzer (1999). "Caveolae require intact VAMP for targeted transport in vascular endothelium." Am J Physiol 277(6 Pt 2): H2222-32.

McNally, E. M., E. de Sa Moreira, et al. (1998). "Caveolin-3 in muscular dystrophy." Hum Mol Genet 7(5): 871-7.

Meili, R. and R. A. Firtel (2003). "Two poles and a compass." Cell 114(2): 153-6.

Michel, J. B., O. Feron, et al. (1997). "Reciprocal regulation of endothelial nitric-oxide synthase by Ca2+-calmodulin and caveolin." J Biol Chem 272(25): 15583-6. 
Minetti, C., F. Sotgia, et al. (1998). "Mutations in the caveolin-3 gene cause autosomal dominant limb-girdle muscular dystrophy." Nat Genet 18(4): 365-8.

Monier, S., R. G. Parton, et al. (1995). "VIP21-caveolin, a membrane protein constituent of the caveolar coat, oligomerizes in vivo and in vitro." Mol. Biol. Cell 6: 911927.

Monier, S., R. G. Parton, et al. (1995). "VIP21-caveolin, a membrane protein constituent of the caveolar coat, oligomerizes in vivo and in vitro." Mol Biol Cell 6(7): 91127.

Mora, R., V. L. Bonilha, et al. (1999). "Caveolin-2 localizes to the golgi complex but redistributes to plasma membrane, caveolae, and rafts when co-expressed with caveolin-1." J Biol Chem 274(36): 25708-17.

Mundy, D. I., T. Machleidt, et al. (2002). "Dual control of caveolar membrane traffic by microtubules and the actin cytoskeleton." J Cell Sci 115(Pt 22): 4327-39.

Mustata, T. and V. Rusu (1998). "[Mechanotransduction and tensegrity (I)]." Rev Med Chir Soc Med Nat Iasi 102(3-4): 25-35.

Navarro, A., B. Anand-Apte, et al. (2004). "A role for caveolae in cell migration." Faseb J 18(15): 1801-11.

Nobes, C. D. and A. Hall (1995). "Rho, rac and cdc42 GTPases: regulators of actin structures, cell adhesion and motility." Biochem Soc Trans 23(3): 456-9.

Noegel, A., W. Witke, et al. (1987). "Calcium-sensitive non-muscle alpha-actinin contains EF-hand structures and highly conserved regions." FEBS Lett 221(2): 391-6.

Oh, P. and J. E. Schnitzer (2001). "Segregation of heterotrimeric G proteins in cell surface microdomains. G(q) binds caveolin to concentrate in caveolae, whereas G(i) and G(s) target lipid rafts by default." Mol Biol Cell 12(3): 685-98.

Okada, S. S., J. E. Tomaszewski, et al. (1995). "Migrating vascular smooth muscle cells polarize cell surface urokinase receptors after injury in vitro." Exp Cell Res 217(1): 180-7.

Okamoto, T., A. Schlegel, et al. (1998). "Caveolins, A family of scaffolding proteins for organizing "pre-assembled signaling complexes" at the plasma membrane." $\mathrm{J}$. Biol. Chem., (Mini-review) 273: 5419-5422.

O'Toole, T. E., Y. Katagiri, et al. (1994). "Integrin cytoplasmic domains mediate insideout signal transduction." J Cell Biol 124(6): 1047-59.

Palade, G. E. (1953). "Fine structure of blood capillaries." J. Appl. Physics 24: 1424. 
Palade, G. E. and R. R. Bruns (1968). "Structural modulations of plasmalemmal vesicles." J Cell Biol 37(3): 633-49.

Palecek, S. P., A. Huttenlocher, et al. (1998). "Physical and biochemical regulation of integrin release during rear detachment of migrating cells." J Cell Sci 111 ( Pt 7): $929-40$.

Parat, M. O., B. Anand-Apte, et al. (2003). "Differential caveolin-1 polarization in endothelial cells during migration in two and three dimensions." Mol Biol Cell 14(8): 3156-68.

Parat, M. O. and P. L. Fox (2001). "Palmitoylation of caveolin-1 in endothelial cells is post-translational but irreversible." J Biol Chem 276(19): 15776-82.

Pardo, J. V., J. D. Siliciano, et al. (1983). "Vinculin is a component of an extensive network of myofibril-sarcolemma attachment regions in cardiac muscle fibers." $\underline{\mathrm{J}}$ Cell Biol 97(4): 1081-8.

Park, D. S., H. Lee, et al. (2002). "Caveolin-1-deficient mice show accelerated mammary gland development during pregnancy, premature lactation, and hyperactivation of the Jak-2/STAT5a signaling cascade." Mol Biol Cell 13(10): 3416-30.

Park, D. S., S. E. Woodman, et al. (2002). "Caveolin-1/3 double-knockout mice are viable, but lack both muscle and non-muscle caveolae, and develop a severe cardiomyopathic phenotype." Am J Pathol 160(6): 2207-17.

Parolini, I., M. Sargiacomo, et al. (1999). "Expression of caveolin-1 is required for the transport of caveolin-2 to the plasma membrane. Retention of caveolin-2 at the level of the golgi complex." J Biol Chem 274(36): 25718-25.

Pavalko, F. M., C. A. Otey, et al. (1991). "Alpha-actinin: a direct link between actin and integrins." Biochem Soc Trans 19(4): 1065-9.

Pelham, R. J., Jr. and Y. Wang (1999). "High resolution detection of mechanical forces exerted by locomoting fibroblasts on the substrate." Mol Biol Cell 10(4): 935-45.

Pelkmans, L., J. Kartenbeck, et al. (2001). "Caveolar endocytosis of simian virus 40 reveals a new two-step vesicular-transport pathway to the ER." Nat Cell Biol 3(5): 473-83.

Pelkmans, L., D. Puntener, et al. (2002). "Local actin polymerization and dynamin recruitment in SV40-induced internalization of caveolae." Science 296(5567): 535-9.

Pelkmans, L. and M. Zerial (2005). "Kinase-regulated quantal assemblies and kiss-andrun recycling of caveolae." Nature 436(7047): 128-33. 
Peters, K.-R., W. Carley, et al. (1985). "Endothelial plasmalemmal vesicles have a characteristic striped bipolar surface structure." J. Cell Biol. 101: 2233-2238.

Peters, K. R., W. W. Carley, et al. (1985). "Endothelial plasmalemmal vesicles have a characteristic striped bipolar surface structure." J Cell Biol 101(6): 2233-8.

Pike, L. J. and L. Casey (1996). "Localization and turnover of phosphatidylinositol 4,5bisphosphate in caveolin-enriched membrane domains." J. Biol. Chem. 271: 26453-26456.

Raftopoulou, M. and A. Hall (2004). "Cell migration: Rho GTPases lead the way." Dev Biol 265(1): 23-32.

Rajfur, Z., P. Roy, et al. (2002). "Dissecting the link between stress fibres and focal adhesions by CALI with EGFP fusion proteins." Nat Cell Biol 4(4): 286-93.

Razani, B., T. P. Combs, et al. (2002). "Caveolin-1-deficient mice are lean, resistant to diet-induced obesity, and show hypertriglyceridemia with adipocyte abnormalities." J Biol Chem 277(10): 8635-47.

Razani, B., J. A. Engelman, et al. (2001). "Caveolin-1 null mice are viable but show evidence of hyperproliferative and vascular abnormalities." J Biol Chem 276(41): 38121-38.

Razani, B., A. Schlegel, et al. (2001). "Caveolin-1, a putative tumour suppressor gene." Biochem Soc Trans 29(Pt 4): 494-9.

Razani, B., X. B. Wang, et al. (2002). "Caveolin-2-deficient mice show evidence of severe pulmonary dysfunction without disruption of caveolae." Mol Cell Biol 22(7): 2329-44.

Rid, R., N. Schiefermeier, et al. (2005). "The last but not the least: the origin and significance of trailing adhesions in fibroblastic cells." Cell Motil Cytoskeleton 61(3): 161-71.

Ridley, A. J., M. A. Schwartz, et al. (2003). "Cell migration: integrating signals from front to back." Science 302(5651): 1704-9.

Rohlich, P. and A. C. Allison (1976). "Oriented pattern of membrane-associated vesicles in fibroblasts." J Ultrastruct Res 57(1): 94-103.

Rosengren, B. I., A. Rippe, et al. (2006). "Transvascular protein transport in mice lacking endothelial caveolae." Am J Physiol Heart Circ Physiol.

Rothberg, K. G., J. E. Heuser, et al. (1992). "Caveolin, a protein component of caveolae membrane coats." Cell 68: 673-682. 
Rothberg, K. G., J. E. Heuser, et al. (1992). "Caveolin, a protein component of caveolae membrane coats." Cell 68(4): 673-82.

Rottner, K., A. Hall, et al. (1999). "Interplay between Rac and Rho in the control of substrate contact dynamics." Curr Biol 9(12): 640-8.

Sargiacomo, M., P. E. Scherer, et al. (1995). "Oligomeric structure of caveolin: implications for caveolae membrane organization." Proc Natl Acad Sci U S A 92(20): 9407-11.

Sargiacomo, M., M. Sudol, et al. (1993). "Signal transducing molecules and glycosylphosphatidylinositol-linked proteins form a caveolin-rich insoluble complex in MDCK cells." J Cell Biol 122(4): 789-807.

Satish, L., H. C. Blair, et al. (2005). "Interferon-inducible protein 9 (CXCL11)-induced cell motility in keratinocytes requires calcium flux-dependent activation of mucalpain." Mol Cell Biol 25(5): 1922-41.

Sawada, H., H. Ishikawa, et al. (1978). "High resolution scanning electron microscopy of frog sartorius muscle." Tissue Cell 10(1): 179-90.

Schaller, M. D. and J. T. Parsons (1994). "Focal adhesion kinase and associated proteins." Curr Opin Cell Biol 6(5): 705-10.

Schaller, M. D. and J. T. Parsons (1995). "pp125FAK-dependent tyrosine phosphorylation of paxillin creates a high-affinity binding site for Crk." Mol Cell Biol 15(5): 2635-45.

Scherer, P. E., R. Y. Lewis, et al. (1997). "Cell-type and tissue-specific expression of caveolin-2. Caveolins 1 and 2 co-localize and form a stable hetero-oligomeric complex in vivo." J Biol Chem 272(46): 29337-46.

Scherer, P. E., T. Okamoto, et al. (1996). "Identification, sequence, and expression of caveolin-2 defines a caveolin gene family." Proc Natl Acad Sci U S A 93(1): 1315.

Scherer, P. E., Z. Tang, et al. (1995). "Caveolin isoforms differ in their N-terminal protein sequence and subcellular distribution. Identification and epitope mapping of an isoform-specific monoclonal antibody probe." J Biol Chem 270(27): 16395401.

Schlaepfer, D. D. and T. Hunter (1996). "Evidence for in vivo phosphorylation of the Grb2 SH2-domain binding site on focal adhesion kinase by Src-family proteintyrosine kinases." Mol Cell Biol 16(10): 5623-33.

Schubert, W., P. G. Frank, et al. (2001). "Caveolae-deficient endothelial cells show defects in the uptake and transport of albumin in vivo." J Biol Chem 276(52): 48619-22. 
Schubert, W., P. G. Frank, et al. (2002). "Microvascular hyperpermeability in caveolin-1 (-/-) knock-out mice. Treatment with a specific nitric-oxide synthase inhibitor, LNAME, restores normal microvascular permeability in Cav-1 null mice." J Biol Chem 277(42): 40091-8.

Segall, J. E., S. Tyerech, et al. (1996). "EGF stimulates lamellipod extension in metastatic mammary adenocarcinoma cells by an actin-dependent mechanism." Clin Exp Metastasis 14(1): 61-72.

Sessa, W. C. (2005). "Regulation of endothelial derived nitric oxide in health and disease." Mem Inst Oswaldo Cruz 100 Suppl 1: 15-8.

Shajahan, A. N., B. K. Timblin, et al. (2004). "Role of Src-induced dynamin-2 phosphorylation in caveolae-mediated endocytosis in endothelial cells." J Biol Chem 279(19): 20392-400.

Shajahan, A. N., C. Tiruppathi, et al. (2004). "Gbetagamma activation of Src induces caveolae-mediated endocytosis in endothelial cells." J Biol Chem 279(46): 4805562.

Sharma, C. P., R. M. Ezzell, et al. (1995). "Direct interaction of filamin (ABP-280) with the beta 2-integrin subunit CD18." J Immunol 154(7): 3461-70.

Shaul, P. W. and R. G. Anderson (1998). "Role of plasmalemmal caveolae in signal transduction." Am J Physiol 275(5 Pt 1): L843-51.

Shaul, P. W., E. J. Smart, et al. (1996). "Acylation targets emdothelial nitric-oxide synthase to plasmalemmal caveolae." J Biol Chem 271(11): 6518-22.

Simionescu, M., N. Simionescu, et al. (1974). "Morphometric data on the endothelium of blood capillaries." J Cell Biol 60(1): 128-52.

Smart, E., Y.-S. Ying, et al. (1994). "Caveolin moves from caveolae to the Golgi apparatus in response to cholesterol oxidation." J. Cell Biol. 127: 1185-1197.

Smilenov, L. B., A. Mikhailov, et al. (1999). "Focal adhesion motility revealed in stationary fibroblasts." Science 286(5442): 1172-4.

Song, K. S., P. E. Scherer, et al. (1996). "Expression of caveolin-3 in skeletal, cardiac, and smooth muscle cells. Caveolin-3 is a component of the sarcolemma and cofractionates with dystrophin and dystrophin-associated glycoproteins." J Biol Chem 271(25): 15160-5.

Song, K. S., Z. Tang, et al. (1997). "Mutational analysis of the properties of caveolin-1. A novel role for the C-terminal domain in mediating homo-typic caveolin-caveolin interactions." J Biol Chem 272(7): 4398-403. 
Sonveaux, P., P. Martinive, et al. (2004). "Caveolin-1 expression is critical for vascular endothelial growth factor-induced ischemic hindlimb collateralization and nitric oxide-mediated angiogenesis." Circ Res 95(2): 154-61.

Sowa, G., M. Pypaert, et al. (2003). "The phosphorylation of caveolin-2 on serines 23 and 36 modulates caveolin-1-dependent caveolae formation." Proc Natl Acad Sci U S A 100(11): 6511-6.

Stahlhut, M. and B. van Deurs (2000). "Identification of filamin as a novel ligand for caveolin-1: evidence for the organization of caveolin-1-associated membrane domains by the actin cytoskeleton." Mol Biol Cell 11(1): 325-37.

Stan, R. V. (2005). "Structure of caveolae." Biochim Biophys Acta 1746(3): 334-48.

Tagawa, A., A. Mezzacasa, et al. (2005). "Assembly and trafficking of caveolar domains in the cell: caveolae as stable, cargo-triggered, vesicular transporters." J Cell Biol 170(5): 769-79.

Tang, S., K. G. Morgan, et al. (1997). "Requirement for protein kinase C theta for cell cycle progression and formation of actin stress fibers and filopodia in vascular endothelial cells." J. Biol. Chem. 272: 28704-28711.

Tang, Z., P. E. Scherer, et al. (1996). "Molecular cloning of caveolin-3, a novel member of the caveolin gene family expressed predominantly in muscle." J Biol Chem 271(4): 2255-61.

Thomsen, P., K. Roepstorff, et al. (2002). "Caveolae are highly immobile plasma membrane microdomains, which are not involved in constitutive endocytic trafficking." Mol Biol Cell 13(1): 238-50.

Torgersen, M. L., G. Skretting, et al. (2001). "Internalization of cholera toxin by different endocytic mechanisms." J Cell Sci 114(Pt 20): 3737-47.

Tozer, E. C., P. E. Hughes, et al. (1996). "Ligand binding and affinity modulation of integrins." Biochem Cell Biol 74(6): 785-98.

Travis, J. (1993). "Cell biologists explore 'tiny caves'." Science 262: 1208-1209.

Turner, C. E., J. R. Glenney, Jr., et al. (1990). "Paxillin: a new vinculin-binding protein present in focal adhesions." J Cell Biol 111(3): 1059-68.

Turner, C. E. and J. T. Miller (1994). "Primary sequence of paxillin contains putative SH2 and SH3 domain binding motifs and multiple LIM domains: identification of a vinculin and pp125Fak-binding region." J Cell Sci 107 ( Pt 6): 1583-91.

Uehara, K. and M. Miyoshi (2002). "Localization of caveolin-3 in the sinus endothelial cells of the rat spleen." Cell Tissue Res 307(3): 329-36. 
Vey, M., S. Pilkuhn, et al. (1996). "Subcellular localization of the scrapie prion proteins in caveolae-like membranous domains." Proc. Natl. Acad. Sci. USA 93: 1494514949.

Vinogradova, O., A. Velyvis, et al. (2002). "A structural mechanism of integrin alpha(IIb)beta(3) "inside-out" activation as regulated by its cytoplasmic face." Cell 110(5): 587-97.

Volonte, D., F. Galbiati, et al. (2001). "Cellular stress induces the tyrosine phosphorylation of caveolin-1 (Tyr(14)) via activation of p38 mitogen-activated protein kinase and c-Src kinase. Evidence for caveolae, the actin cytoskeleton, and focal adhesions as mechanical sensors of osmotic stress." J Biol Chem 276(11): 8094-103.

Wang, X. B., H. Lee, et al. (2004). "Tyrosine phosphorylation of caveolin-2 at residue 27: differences in the spatial and temporal behavior of phospho-Cav-2 (pY19 and pY27)." Biochemistry 43(43): 13694-706.

Wary, K. K., i. A. Mariott, et al. (1998). "A requirement for caveolin-1 and associated kinase Fyn in integrin signaling and anchorage-dependent cell growth." Cell 94: 625-634.

Wary, K. K., A. Mariotti, et al. (1998). "A requirement for caveolin-1 and associated kinase Fyn in integrin signaling and anchorage-dependent cell growth." Cell 94(5): 625-34.

Webb, D. J., K. Donais, et al. (2004). "FAK-Src signalling through paxillin, ERK and MLCK regulates adhesion disassembly." Nat Cell Biol 6(2): 154-61.

Webb, D. J., J. T. Parsons, et al. (2002). "Adhesion assembly, disassembly and turnover in migrating cells -- over and over and over again." Nat Cell Biol 4(4): E97-100.

Wei, Y., X. Yang, et al. (1999). "A role for caveolin and the urokinase receptor in integrin-mediated adhesion and signaling." J Cell Biol 144(6): 1285-94.

Williams, T. M. and M. P. Lisanti (2004). "The caveolin proteins." Genome Biol 5(3): 214.

Woodman, S. E., A. W. Ashton, et al. (2003). "Caveolin-1 knockout mice show an impaired angiogenic response to exogenous stimuli." Am J Pathol 162(6): 205968.

Woodman, S. E., M. W. Cheung, et al. (2004). "Urogenital alterations in aged male caveolin-1 knockout mice." J Urol 171(2 Pt 1): 950-7.

Woodman, S. E., D. S. Park, et al. (2002). "Caveolin-3 knock-out mice develop a progressive cardiomyopathy and show hyperactivation of the p42/44 MAPK cascade." J Biol Chem 277(41): 38988-97. 
Woodman, S. E., F. Sotgia, et al. (2004). "Caveolinopathies: mutations in caveolin-3 cause four distinct autosomal dominant muscle diseases." Neurology 62(4): 53843.

Yamada, E. (1955). "The fine structure of the gall bladder epithelium of the mouse." $\underline{J}$ Biophys Biochem Cytol 1(5): 445-58.

Yamamoto, M., Y. Toya, et al. (1998). "Caveolin is an activator of insulin receptor signaling." J Biol Chem 273(41): 26962-8.

Yao, Q., J. Chen, et al. (2005). "Caveolin-1 interacts directly with dynamin-2." J Mol Biol 348(2): 491-501.

Yu, J., S. Bergaya, et al. (2006). "Direct evidence for the role of caveolin-1 and caveolae in mechanotransduction and remodeling of blood vessels." J Clin Invest 116(5): 1284-91.

Zamir, E. and B. Geiger (2001). "Components of cell-matrix adhesions." J Cell Sci 114(Pt 20): 3577-9.

Zicha, D., G. A. Dunn, et al. (1991). "A new direct-viewing chemotaxis chamber." $\underline{\mathrm{J} \text { Cell }}$ Sci 99 ( Pt 4): 769-75.

Zundel, W., L. M. Swiersz, et al. (2000). "Caveolin 1-mediated regulation of receptor tyrosine kinase-associated phosphatidylinositol 3-kinase activity by ceramide." Mol Cell Biol 20(5): 1507-14. 